\title{
Joint Invariant Signatures
}

\author{
Peter J. Olver ${ }^{\dagger}$ \\ School of Mathematics \\ University of Minnesota \\ Minneapolis, MN 55455 \\ U.S.A. \\ olver@umn.edu \\ http://www. math.umn.edu/ olver
}

Dedicated to the memory of Gian-Carlo Rota

\begin{abstract}
A new, algorithmic theory of moving frames is applied to classify joint invariants and joint differential invariants of transformation groups. Equivalence and symmetry properties of submanifolds are completely determined by their joint signatures, which are parametrized by a suitable collection of joint invariants and/or joint differential invariants. A variety of fundamental geometric examples are developed in detail. Applications to object recognition problems in computer vision and the design of invariant numerical approximations are indicated.
\end{abstract}

$\dagger$ Supported in part by NSF Grant DMS 98-03154.

August 7, 2000 


\section{Introduction.}

This paper is devoted to the algorithmic classification of joint invariants and joint differential invariants for transformation groups. Applications to equivalence and symmetry properties of submanifolds, especially curves and surfaces, are discussed in detail. Our approach relies on a new, practical extension of the Cartan theory of moving frames, $[\mathbf{1 4}, \mathbf{2 2}, \mathbf{2 1}, \mathbf{2 4}]$, which was recently formulated by the author and M. Fels, $[\mathbf{1 8}, \mathbf{1 9}, \mathbf{2 0}]$. The moving frame method provides a direct route to what Weyl, in the algebraic context, calls the "First Main Theorem" for a transformation group, [43], namely the classification of all its joint invariants as well as new classifications of joint differential invariants.

Recent applications of the moving frame method in computer vision, $[\mathbf{1 7}, \mathbf{1 3}]$, provide additional impetus for this general program. In $[\mathbf{5}, \mathbf{6}, \mathbf{7}, \mathbf{1 3}, \mathbf{3 6}]$, differential invariant signature manifolds were introduced for invariantly characterizing object boundaries and detecting symmetries. One significant difficulty has been the noise sensitivity of standard differential invariants owing to their dependence on high order derivatives. An alternative approach, $[\mathbf{8}, \mathbf{1 1}, \mathbf{3 0}, \mathbf{3 8}, \mathbf{3 9}]$, is to employ "noise-resistant" joint differential invariants, or, as they are known in the computer vision literature, "semi-differential invariants", which may depend on lower order derivatives. Dhooghe, [16], initiated the systematic classification of joint differential invariants, based on algebraic identities as well as Lie algebraic methods; in [18] we showed how to apply moving frame methods, thereby enabling us to handle a wide variety of new and interesting examples. The approximation of higher order differential invariants by joint differential invariants and, generally, ordinary joint invariants leads to fully invariant finite difference numerical schemes, first proposed in $[\mathbf{1 2}, \mathbf{1 3}]$. Applications of these results to the construction of invariant numerical algorithms and the theory of geometric integration, $[\mathbf{1 0}, \mathbf{2 8}]$, will be developed in a subsequent paper.

In this paper, we systematically build on the preliminary investigations announced in [18]. We show how the method of moving frames can be readily used to compute complete systems of joint differential invariants, and illustrate with examples of direct interest in image processing. The particular groups used to illustrate the basic techniques are the classical Euclidean, equi-affine, affine and projective groups acting on $n$-dimensional space, with special emphasis on the cases of curves and surfaces in two and three dimensions.

The moving frame theory also shows precisely which joint differential invariants are required to parametrize a signature that will uniquely characterize the original submanifold up to group transformations. The more points on the curve or surface that the joint invariants depend on, the lower the order of differentiation required to compute the signature, thereby reducing the noise sensitivity of the resulting signature. With enough points, one can completely characterize the submanifold by joint invariants alone, and thereby entirely avoid differentiation. The trade-off is that the more points used, the higher the dimension of the signature set, and so the more data that must be computed. However, in all examples, there is only one fundamental joint invariant, and the signature set is obtained by assembling the joint invariant, depending on various points on the submanifold, in certain well-prescribed combinations.

For example, suppose we wish to characterize planar curves up to a Euclidean motion. The "classical" signature relies on the fact that a planar curve $C \subset \mathbb{R}^{2}$ is uniquely characterized up to a Euclidean transformation by its curvature as a function of arc length, $\kappa(s)$, 


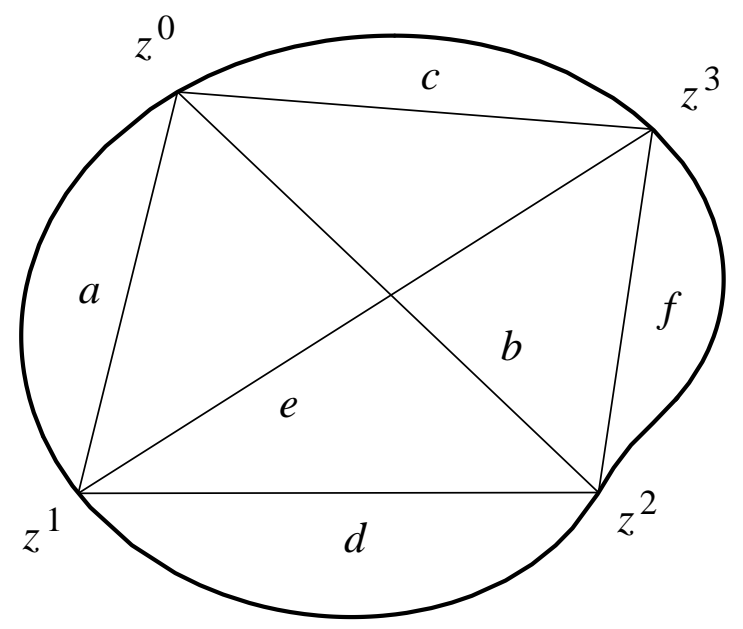

Figure 1. Four-Point Euclidean Curve Invariants.

cf. [24]. To eliminate the ambiguity caused by the choice of initial point from which to measure the arc length $s$ (an important consideration in occlusion problems), we proposed to use the signature curve $\mathcal{S}=\mathcal{S}(C)$, which is parameterized by the two functions $\kappa, \kappa_{s}$, to characterize $C,[\mathbf{1 3}]$. Both signatures require computing second and, in the latter version, third order derivatives. A Euclidean joint invariant signature that does not require differentiation can be obtained as follows. First, the basic joint Euclidean invariant is the distance $\|z-w\|$ between two points. To create a Euclidean signature set based entirely on joint invariants, we must use four points $z^{0}, z^{1}, z^{2}, z^{3}$ on our curve $C \subset \mathbb{R}^{2}$. As illustrated in Figure 1, there are six different interpoint distance invariants

$$
\begin{array}{lll}
a=\left\|z^{1}-z^{0}\right\|, & b=\left\|z^{2}-z^{0}\right\|, & c=\left\|z^{3}-z^{0}\right\|, \\
d=\left\|z^{2}-z^{1}\right\|, & e=\left\|z^{3}-z^{1}\right\|, & f=\left\|z^{3}-z^{2}\right\| .
\end{array}
$$

As $z^{0}, z^{1}, z^{2}, z^{3}$ move on the curve, the distances (1.1) parametrize the joint signature $\widehat{\mathcal{S}}=\widehat{\mathcal{S}}(C)$, which, according to our general theory, uniquely characterizes the curve $C$ up to Euclidean motion. The important fact is that this signature requires no differentiation, and so is not at all sensitive to noisy image data - unlike the curvature-based signature $\mathcal{S}$, the joint signature $\widehat{\mathcal{S}}$ is no more noisy than the original curve. Since $a, b, c, d, e, f$ form six functions of four variables (the four points on the curve), there are two syzygies (functional relations)

$$
\Phi_{1}(a, b, c, d, e, f)=0, \quad \Phi_{2}(a, b, c, d, e, f)=0,
$$

among them. One of these is the Cayley-Menger syzygy

$$
\operatorname{det}\left|\begin{array}{ccc}
2 a^{2} & a^{2}+b^{2}-d^{2} & a^{2}+c^{2}-e^{2} \\
a^{2}+b^{2}-d^{2} & 2 b^{2} & b^{2}+c^{2}-f^{2} \\
a^{2}+c^{2}-e^{2} & b^{2}+c^{2}-f^{2} & 2 c^{2}
\end{array}\right|=0
$$


which is valid for all possible configurations of the four points, and is a consequence of their coplanarity, cf. $[\mathbf{2}, \mathbf{2 9}]$. This identity is universal, meaning that it is independent of the particular curve $C$. Therefore, the second syzygy in (1.2) is curve-dependent and serves to effectively characterize the joint invariant signature. Euclidean symmetries of the curve, both continuous and discrete, are characterized by the signature; for example, the number of symmetries is equal to the "index" of the signature, meaning the number of points in the original curve that map to a single, generic, point in $\mathcal{S}$. Further details appear in Example 8.2 below.

In this paper, we will systematically develop the general theory underlying this particular construction, in an algorithmic form that can be applied to arbitrary Lie transformation groups, acting on curves, surfaces, or more general submanifolds. Moreover, we investigate in detail some of the principal applications, including planar curves, and space curves and surfaces, under Euclidean, equi-affine and projective transformation groups.

\section{Introduction to Moving Frames.}

We begin with a summary of the general, algorithmic moving frame method developed in [19]. We assume that $G$ is an $r$-dimensional Lie group ${ }^{\dagger}$ acting smoothly on an $m$ dimensional manifold $M$. In most computer vision applications, $M=\mathbb{R}^{2}$ or $\mathbb{R}^{3}$, and $G$ is a group of geometrical significance, e.g., the Euclidean group, equi-affine group, projective group, etc. However, the advantage of our new approach is that the methods are applicable to completely general Lie group actions.

Let us introduce some basic terminology. The isotropy subgroup of a point $z \in M$ is $G_{z}=\{g \in G \mid g \cdot z=z\}$. The group is said to act freely if $G_{z}=\{e\}$ for all $z \in M$. The action is called locally free if each $G_{z}$ forms a discrete subgroup of $G$; this is equivalent to the condition that the orbits all have dimension $r=\operatorname{dim} G$. The global isotropy subgroup

$$
G_{M}^{*}=\{g \in G \mid g \cdot z=z \text { for all } z \in M\}
$$

is a normal subgroup of $G$ consisting of all group elements that have completely trivial action on $M$. The action is effective if $G_{M}^{*}=\{e\}$, and locally effective if $G_{M}^{*}$ is a discrete subgroup. Any non-effective action can be converted into an equivalent effective action of the quotient Lie group $G / G_{M}^{*}$ that essentially coincides with that of $G$, cf. [32]. The group acts regularly if all its orbits have the same dimension and, moreover, each point $z \in M$ admits a system of neighborhoods having pathwise connected intersection with each group orbit. The regularity condition avoids pathologies such as the irrational flow on the torus, where the orbits return arbitrarily close to themselves.

We shall formulate Cartan's concept of a moving frame ("repère mobile"), [14], in a precise and general manner. The fundamental definition, as motivated by earlier work of Griffiths, $[\mathbf{2 2}]$, and Green, $[\mathbf{2 1}]$, frees the theory from reliance on any sort of frame bundle - an artifice that does not appear as naturally in non-geometrical situations. See [19] for more discussion of this point.

$\dagger$ The moving frame methods can be extended to infinite-dimensional pseudo-group actions, [18], but, for simplicity, we will only consider the finite-dimensional case here. 
Definition 2.1. A moving frame is a smooth, $G$-equivariant map $\rho: M \rightarrow G$.

In general, if $G$ acts on spaces $M$ and $N$, a map $\varphi: M \rightarrow N$ is called equivariant provided $\varphi(g \cdot z)=g \cdot \varphi(z)$ for all $g \in G, z \in M$. In the case of a moving frame, there are two natural actions of $G$ on itself — by left multiplication $h \mapsto g \cdot h$ or by right multiplication $h \mapsto h \cdot g^{-1}$ — leading to the concepts of left and right moving frames. Classical geometry relies on left moving frames, but we shall sometimes find that a right moving frame is more convenient to compute. Fortunately, there is a simple connection between the two: if $\rho(z)$ is any right-equivariant moving frame then $\widetilde{\rho}(z)=\rho(z)^{-1}$ is a left-equivariant moving frame and conversely. The following simple theorem provides immediate necessary and sufficient conditions for the local existence of a moving frame.

Theorem 2.2. A moving frame exists in a neighborhood of a point $z \in M$ if and only if $G$ acts freely and regularly near $z$.

Of course, most interesting group actions, including all the geometrical examples to be analyzed here, are not free, and therefore do not admit moving frames in the sense of Definition 2.1. For example, the Euclidean group $\mathrm{E}(2)$ does not act freely on $M=\mathbb{R}^{2}$ since all rotations centered at a given point will fix that point. The problem is, of course, that the underlying manifold does not have a high enough dimension to admit $r$-dimensional orbits - a Lie group may only act freely on manifolds of dimension at least as large as that of the group.

There are two important methods for converting a non-free (but effective) action into a free action. The first is to look at the joint or product action of $G$ on several copies of $M$. For example, the action of $\mathrm{E}(2)$ on the Cartesian product $M^{\times 2}=M \times M$ is free on the off-diagonal part $\mathcal{V}=\left\{z^{1} \neq z^{2}\right\} \subset M^{\times 2}$ because the identity is the only Euclidean transformation that fixes two distinct points. The extension of a group action to a Cartesian product leads, naturally, to joint invariants, and the applications of our moving frame methods to this situation will be discussed in Section 3.

The second method is to prolong the group action to the jet spaces coordinatized by the derivatives (i.e., jets) of submanifolds. Jet space is the natural setting for the traditional moving frames that appear in geometry, and lead to differential invariants; details will appear in Section 4 below. Combining the two methods of prolongation and product will lead to joint differential invariants and joint invariant signatures, whose analysis is the main goal of this paper. Complete results appear starting in Section 7.

Given a free group action, the construction of a moving frame is based on Cartan's method of normalization, [14]; an early version can be found in Killing, [26]. The key observation, $[\mathbf{1 8}, \mathbf{1 9}]$, is that normalization amounts to the choice of a cross-section to the group orbits.

Definition 2.3. Let $G$ be a Lie group that acts regularly on the $m$-dimensional manifold $M$ with $s$-dimensional orbits. A cross-section is an $(m-s)$-dimensional submanifold $K \subset X$ that intersects each orbit transversally in exactly one point.

Remark: Typically, one may only be able to construct a local cross-section $K$, meaning that it may intersect the orbits in either one or no points. However, we can trivially convert 


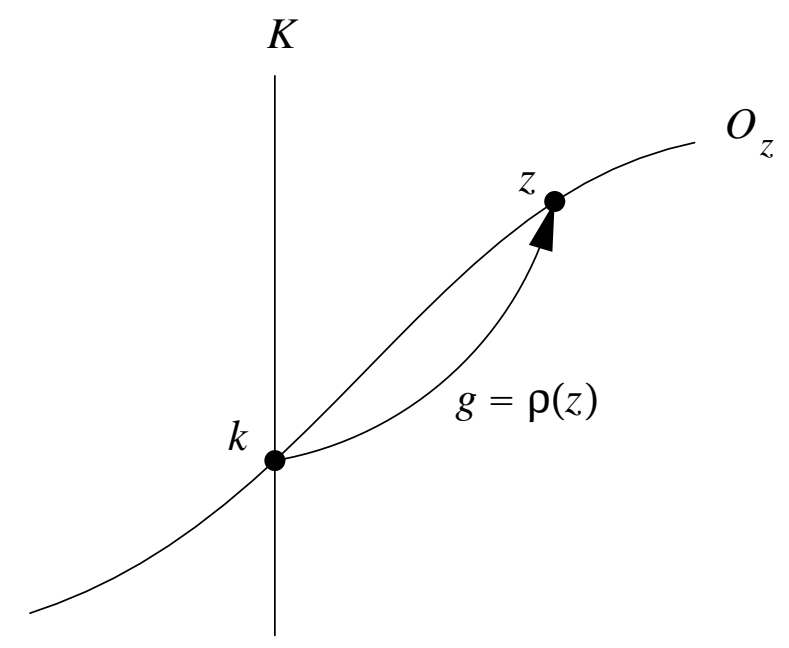

Figure 2. Moving Frame Associated with Cross-Section.

a local cross-section $K$ into a global cross-section by replacing $M$ by the open submanifold $\widetilde{M}=G \cdot K$ consisting of all the orbits that do intersect $K$, and so our restriction to global cross-sections is not essential. Furthermore, we shall only consider cross-sections for free group actions in this paper, in which case $K$ has dimension $m-r$, where $r=\operatorname{dim} G$.

The elements $k \in K$ of the cross-section can be viewed as "canonical forms" for general points $z \in M$, and hence their coordinates are invariant functions. Any regular Lie group action admits many local cross-sections; the most important are the coordinate cross-sections

$$
K=\left\{z_{1}=c_{1}, \ldots, z_{r}=c_{r}\right\},
$$

obtained by equating $r$ of the coordinates in $z=\left(z_{1}, \ldots, z_{m}\right)$ (which, by relabeling if necessary can be taken to be the first $r$ coordinates) to constants. Of course, any crosssection can be (locally) converted into a coordinate cross-section by a suitable choice of local coordinates.

Theorem 2.4. Let $G$ act freely, regularly on $M$, and let $K$ be a cross-section to the group orbits. Given $z \in M$, let $k=k(z) \in \mathcal{O}_{z} \cap K$ be the unique cross-section element lying in the orbit $\mathcal{O}_{z}=G \cdot z$ through $z$ - the canonical form of $z$. Further, let $g=\rho(z)$ be the unique group element such that $g \cdot z=k$. Then the resulting map $\rho: M \rightarrow G$ is a right moving frame for the group action.

Indeed, every right moving frame has this form - the corresponding cross-section is just the inverse image of the identity: $K=\rho^{-1}\{e\}$. The moving frame associated with the chosen cross-section is found by solving the normalization equations

$$
g \cdot z=k \in K \quad \text { for } \quad g=\rho(z) .
$$

In particular, if we choose the coordinate cross-section (2.2), and write out the group transformations in coordinates as $w(g, z)=g \cdot z$, then the right moving frame is obtained 
by solving the $r=\operatorname{dim} G$ implicit equations

$$
w_{1}(g, z)=c_{1}, \quad \cdots \quad w_{r}(g, z)=c_{r},
$$

for the group parameters $g=\left(g_{1}, \ldots, g_{r}\right)$ in terms of the coordinates $z=\left(z_{1}, \ldots, z_{m}\right)$.

Remark: The corresponding left moving frame can be found by inversion $\widetilde{\rho}(z)=$ $\rho(z)^{-1}$. A direct method is to use the explicit formulae $\widetilde{w}(g, z)=g^{-1} \cdot z$ for the inverse group transformations and solve the associated "inverse" normalization equations

$$
\widetilde{w}_{1}(g, z)=c_{1}, \quad \cdots \quad \widetilde{w}_{r}(g, z)=c_{r} .
$$

The solution $\widetilde{g}=\widetilde{\rho}(z)=\rho(z)^{-1}$ is the unique group element mapping $k \in K$ to $z=\widetilde{g} \cdot k$.

Remark: In practice, one often normalizes "in stages", solving some of the normalization equations for some of the group parameters in terms of the coordinates $z$ and the remaining group parameters, and then substituting these expressions into the remaining normalization equations in order to continue the process.

Given such a right moving frame, the non-constant coordinates of the canonical form $k(z)=\rho(z) \cdot z \in K$ are the fundamental invariants for the group action.

Theorem 2.5. Given a free, regular Lie group action and coordinate cross-section (2.2), let $g=\rho(z)$ denote the solution to the normalization equations (2.4). Then the functions

$$
I_{1}(z)=w_{r+1}(\rho(z), z), \quad \ldots \quad I_{m-r}(z)=w_{m}(\rho(z), z),
$$

obtained by substituting the moving frame formulae into the $m-r$ unnormalized components of $w(g, z)=g \cdot z$ form a complete system of functionally independent invariants.

An important observation is that the moving frame provides a natural way to associate to each function on $M$ a uniquely defined invariant.

Definition 2.6. Let $\rho: M \rightarrow G$ be a right moving frame. The invariantization of a scalar function $F: M \rightarrow \mathbb{R}$ with respect to $\rho$ is the invariant function $I=\iota(F)$ defined by

$$
I(z)=F(\rho(z) \cdot z) .
$$

The value of the invariantization (2.7) at a point $z \in M$ equals the value of $F(k)$ at the canonical form $k=\rho(z) \cdot z \in K$ of $z$. In other words, $I=\iota(F)$ is obtained by restricting $F$ to the cross-section, $I|K=F| K$, and then requiring that $I$ be constant along the orbits. In particular, if $I(z)$ is an invariant, then $\iota(I)=I$. Therefore, the invariantization process defines a projection $\iota: F \mapsto I$, depending on the moving frame, from general functions to invariants.

\section{Classification of Joint Invariants.}

Let us illustrate the basic moving frame constructions in the case of joint actions of classical groups. One outcome will be complete classifications of joint invariants. These 
results are analogous to what Weyl, [43], calls the "First Main Theorem" for the transformation group. However, Weyl's classifications of joint invariants are algebraic - meaning up to polynomial independence. The moving frame classifications are geometric/analytic - meaning up to functional independence - and so may require a much smaller collection of joint invariants. Nevertheless, we find that in the algebraic situations treated in Weyl, the two classifications lead to the same types of joint invariants, although the algebraic context requires more of them. An important observation is the ease with which one constructs a complete system of functionally independent joint invariants, avoiding all of the classical algebraic complications, as well as being readily able to handle much more general situations that are either unknown or cannot be handled by standard algebraic techniques. This distinction between functional and algebraic independence is emphasized by Weyl, [43;p. 30], who states that "... the purely functional part - asserting that the valuesof all invariants are determined by the values of the basic invariants - [is] almost trivial; the essential difficulties lie in the algebraic part only."

Given a Lie group $G$ acting on $M$, we consider the joint action of $G$ on the $(n+1)$-fold Cartesian product $M^{\times(n+1)}=M \times \cdots \times M$ given by

$$
g \cdot\left(z^{0}, \ldots, z^{n}\right)=\left(g \cdot z^{0}, \ldots, g \cdot z^{n}\right), \quad g \in G, \quad z^{0}, \ldots, z^{n} \in M .
$$

An invariant $I\left(z^{0}, \ldots, z^{n}\right)$ of such a Cartesian product action is known as an $(n+1)$-point joint invariant of the original transformation group. Note that any $(n+1)$-point invariant can be viewed as a $k$-point invariant for any $k \geq n+1$ - indeed, in several different ways. For example, a two-point invariant $I\left(z^{0}, z^{1}\right)$ produces potentially six different three-point invariants on $M^{\times 3}$, namely $\widehat{I}\left(z^{0}, z^{1}, z^{2}\right)=I\left(z^{0}, z^{1}\right)$ or $I\left(z^{1}, z^{0}\right)$ or $I\left(z^{0}, z^{2}\right)$ and so on. To avoid this trivial extension, we will usually reserve the term $(n+1)$-point invariant for a joint invariant which does not depend on fewer points.

In many cases, if $G$ acts effectively on $M$, then, for $n \gg 0$ sufficiently large, the product action is free on an open subset of $M^{\times(n+1)}$. However, this is not universally true, as the following example illustrates.

Example 3.1. Let $M=\mathbb{R}^{2} \backslash\{0\}$, with polar coordinates $(r, \theta)$. Consider the oneparameter transformation group

$$
(r, \theta) \longmapsto(r, \theta+r t), \quad t \in G=\mathbb{R} .
$$

The action is clearly effective and locally free; the orbits are the circles centered at the origin. However, the product action is not free on the dense subset

$$
\left\{r^{\mu} / r^{0} \in \mathbb{Q}, \mu=1, \ldots, n\right\} \subset M^{\times(n+1)}
$$

consisting of points whose radii are rational multiples of each other.

Despite the possibility of such pathologies, all of the classical geometrical actions of Lie groups do become free on a suitable dense open subset of a sufficiently large Cartesian product. Consequently, the moving frame method outlined in Section 2 can be applied to such joint actions, and thereby establish complete classifications of joint invariants - a geometric version of the First Main Theorem for the group action. We shall illustrate the basic construction with several important examples. 
Example 3.2. Consider first the equi-affine group $\mathrm{SA}(m)=\mathrm{SL}(m) \ltimes \mathbb{R}^{m}$ acting as volume-preserving affine transformations on multiple copies of $M=\mathbb{R}^{m}$ via

$$
w^{k}=A z^{k}+d, \quad k=0,1,2, \ldots, \quad \text { where } \quad \operatorname{det} A=1, \quad d \in \mathbb{R}^{m} .
$$

We begin by normalizing $w^{0}=0$, which leads to the initial normalization $d=-A z^{0}$ of the translation component of the group element $g=(A, d)$. Substituting the formula for $d$ into (3.2) yields the reduced transformation formulae $w^{k}=A\left(z^{k}-z^{0}\right)$. (This is an example of "normalizing in stages".) If the vectors $z^{1}-z^{0}, \ldots, z^{m}-z^{0}$ are linearly independent, then we can impose the additional normalizations

$$
w^{1}=e_{1}, \quad \ldots \quad w^{m-1}=e_{m-1}, \quad w^{m}=\lambda e_{m} .
$$

Here $\lambda$ is an unspecified scalar, whose value is fixed by the normalization and thereby becomes the first invariant. Solving (3.3) for the group parameters, we find

$$
A=\Lambda Z^{-1}, \quad \text { where } \quad \Lambda=\operatorname{diag}(1,1, \ldots, 1, \lambda)
$$

is a diagonal matrix, and

$$
Z=\left(z^{1}-z^{0} \ldots z^{m}-z^{0}\right)
$$

is the $m \times m$ matrix with the indicated columns. Since $\operatorname{det} A=1$, equation (3.4) implies that

$$
\lambda=\operatorname{det} Z=m ! V(0,1, \ldots, m)
$$

where

$$
V(0,1, \ldots, m)=\frac{1}{m !} \operatorname{det} Z=\frac{(-1)^{m}}{m !} \operatorname{det}\left|\begin{array}{cccc}
z^{0} & z^{1} & \ldots & z^{m} \\
1 & 1 & \ldots & 1
\end{array}\right|
$$

is the (signed) volume of the $m$-dimensional simplex having vertices $z^{0}, z^{1}, \ldots, z^{m}$, which, by our linear independence assumption, is non-zero. Indeed, $\mathrm{SA}(m)$ acts regularly and freely on the dense open subset

$$
\mathcal{V}^{m+1}=\left\{\left(z^{0}, \ldots, z^{m}\right) \in M^{\times(m+1)} \mid V(0,1, \ldots, m) \neq 0\right\} \subset M^{\times(m+1)} .
$$

The induced right moving frame map $\rho: \mathcal{V}^{m+1} \rightarrow \mathrm{SA}(m)$ is explicitly given by

$$
A=\operatorname{diag}(1,1, \ldots, 1, \operatorname{det} Z) Z^{-1}, \quad d=-A z^{0} .
$$

The left moving frame is obtained by inverting the group element, $(\widetilde{A}, \widetilde{b})=(A, b)^{-1}$ :

$$
\widetilde{A}=\operatorname{diag}\left(1,1, \ldots, 1, \frac{1}{\operatorname{det} Z}\right) Z, \quad \widetilde{d}=z^{0}
$$

The simplex volume (3.6) forms the unique $(m+1)$-point joint invariant for the equiaffine group. For more than $m+1$ points, the additional joint invariants are found by substituting the moving frame formulae (3.8) into the components of $w^{k}=A z^{k}+a$ for 


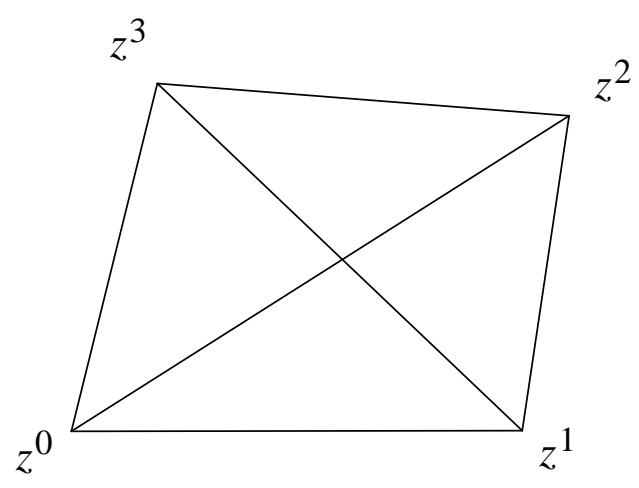

Figure 3. Triangular Area Syzygy.

$k \geq m+1$. A simple application of Cramer's rule gives the complete system of independent joint invariants:

$$
w_{i}^{k}=\left[A\left(z^{k}-z^{0}\right)\right]_{i}= \begin{cases}\frac{V(0,1, \ldots, i-1, k, i+1, \ldots, m)}{V(0,1, \ldots, m)}, & i=1, \ldots, m-1, \\ m ! V(1, \ldots, m-1, k), & i=m .\end{cases}
$$

The Geometric First Main Theorem for the equi-affine group is then an immediate consequence of Theorem 2.5; see Weyl, [43; Theorem 2.7.B], for the algebraic version.

Theorem 3.3. Every joint invariant of the equi-affine group $\mathrm{SA}(m)$ acting on $\mathbb{R}^{m}$ is a function of the simplex volumes

$$
V(I)=V\left(i_{1}, \ldots, i_{m+1}\right)=V\left(z^{i_{1}}, \ldots, z^{i_{m+1}}\right) .
$$

In fact, a generating system of independent invariants is provided by the particular volumes

$$
V(0,1, \ldots, m), \quad V(0,1, \ldots, i-1, i+1, \ldots, m, k), \quad \begin{aligned}
& i=1, \ldots, m, \\
& k \geq m+1 .
\end{aligned}
$$

For example, in the planar case $m=2$, every four-point joint invariant is a function of the three triangular areas $V(0,1,2), V(0,1,3), V(0,2,3)$ indicated in Figure 3. In particular, the remaining triangular area satisfies the evident syzygy

$$
V(1,2,3)=V(0,1,2)+V(0,2,3)-V(0,1,3)
$$

and is hence superfluous for a minimal generating set of invariants.

Example 3.4. The full affine group $\mathrm{A}(m)=\mathrm{GL}(m) \ltimes \mathbb{R}^{m}$ acting on $\mathbb{R}^{m}$ is handled in a very similar fashion. The preliminary normalizations are identical, excepting only 
that we can take $\lambda=1$ in (3.3) since we are no longer restricted to unimodular matrices $A$. Therefore, the left moving frame is simply given by

$$
\widetilde{A}=Z, \quad \widetilde{d}=z^{0} .
$$

The resulting affine joint invariants are ratios of simplex volumes depending on $m+2$ points. We therefore deduce the First Main Theorem for the full affine group.

Theorem 3.5. Every joint invariant of the affine group $\mathrm{A}(m)$ acting on $\mathbb{R}^{m}$ is a function of the simplex volume ratios $V(I) / V(J)$. In fact, a generating system is provided by the particular ratios

$$
\frac{V(0,1, \ldots, i-1, i+1, \ldots, m, k)}{V(0,1, \ldots, m)}, \quad i=1, \ldots, m, \quad k \geq m+1 .
$$

Example 3.6. The Euclidean group $\mathrm{E}(m)=\mathrm{O}(m) \ltimes \mathbb{R}^{m}$ consists of all isometries

$$
w=R z+d, \quad R \in \mathrm{O}(m), \quad d \in \mathbb{R}^{m}, \quad z \in \mathbb{R}^{m},
$$

of Euclidean space. Its orientation-preserving counterpart is the proper Euclidean group $\mathrm{SE}(m)=\mathrm{SO}(m) \ltimes \mathbb{R}^{m}$ generated by rotations and translations. Their joint action is regular and free on the subset $\mathcal{V}^{m+1} \subset M^{\times(m+1)}$ defined in (3.7), because the identity is the only orthogonal transformation that fixes $m$ linearly independent vectors in $\mathbb{R}^{m}$. (Actually, for the proper Euclidean group, freeness occurs on a larger subset since a rotation that fixes $m-1$ linearly independent vectors is necessarily the identity - although there is a reflection that fixes them.)

As in the equi-affine computation, we begin by normalizing $w^{0}=0$ by setting $d=$ $-R z^{0}$. We then normalize the next $m$ vectors $w^{k}=R\left(z^{k}-z^{0}\right), k=1, \ldots, m$, which now only depend on the rotation group parameters. This is accomplished by performing a $Q R$ decomposition, [37], of the matrix $Z$ defined in (3.5). More specifically, we factor $Z=Q U$ where $Q \in \mathrm{SO}(m)$ or $\mathrm{O}(m)$, and $U$ is upper triangular. In the non-oriented case $Q \in \mathrm{O}(\mathrm{m})$, we can require that all the diagonal entries of $U$ be positive, which serves to uniquely specify the orthogonal matrix $Q$. We then normalize $R=Q^{T}$ so that

$$
R Z=U
$$

The solution to (3.13) defines the Euclidean moving frame

$$
\rho:\left(z^{0}, \ldots, z^{m}\right) \quad \longmapsto \quad R=U Z^{-1}, \quad d=-R z^{0} .
$$

The nonzero entries of $U$ provide the $(m+1)$-point Euclidean joint invariants. Since $Q R$ factorization is implemented via the standard Gram-Schmidt algorithm, the entries of $U$ are certain particular combinations of the inner products $\left(z^{i}-z^{0}\right) \cdot\left(z^{j}-z^{0}\right)$ between the various columns of $Z$. Moreover, if $n>m$ then the entries of $R \cdot\left(z^{n}-z^{0}\right)$, which are additional invariants, are also given in terms of such inner products. All such inner products can be written as functions of the distances $\left\|z^{k}-z^{l}\right\|$, and so we have proved a geometric version of the First Main Theorem for the Euclidean group. 


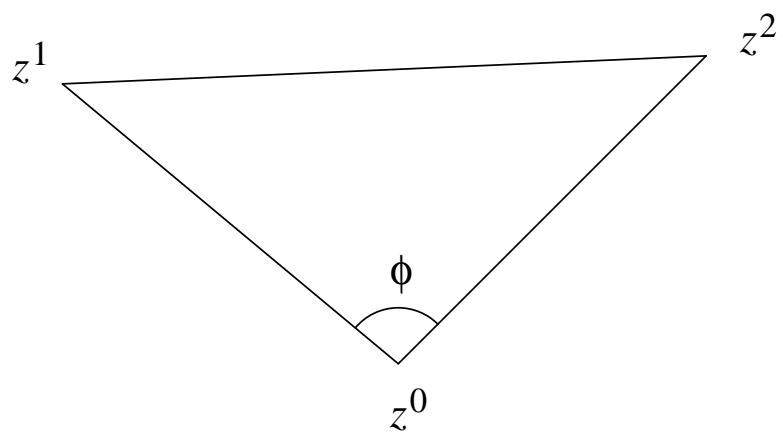

Figure 4. Law of Cosines.

Theorem 3.7. Every joint invariant of the full Euclidean group $\mathrm{E}(m)$ acting on $\mathbb{R}^{m}$ is a function of the interpoint distances $\left\|z^{i}-z^{j}\right\|$.

In the orientation-preserving case, the last diagonal entry of the upper triangular matrix $U$ cannot necessarily be arranged to be positive, although it will be non-zero. The sign of this entry depends on the sign of the volume $V(0,1, \ldots, m)$, which is an orientation-preserving invariant. Therefore, we have the analogous classification result.

Theorem 3.8. Every joint invariant of the special Euclidean group $\mathrm{SE}(m)$ acting on $\mathbb{R}^{m}$ is a function of the interpoint distances $\left\|z^{i}-z^{j}\right\|$ and a single (nonzero) signed simplex volume $V(0,1, \ldots, m)$.

In particular, all other signed simplex volumes are uniquely determined by the interpoint distances and the indicated volume invariant. Furthermore, the squared volume $V(0,1, \ldots, m)^{2}$ can be written as a polynomial in the distances.

Remark: One can use the invariantization procedure to determine explicit syzygies among invariants. As a simple example, consider the three-point invariants of the planar Euclidean group SE(2). According to our normalization, the fundamental invariants are

$$
\begin{aligned}
& y^{0} \longmapsto 0, \quad y^{1} \longmapsto 0, \quad y^{2} \longmapsto\left\|z^{2}-z^{0}\right\| \sin \phi, \\
& v^{0} \longmapsto 0, \quad v^{1} \longmapsto\left\|z^{1}-z^{0}\right\|, \quad v^{2} \longmapsto\left\|z^{2}-z^{0}\right\| \cos \phi,
\end{aligned}
$$

where $\phi$ is the angle between the two chords $z^{2}-z^{0}$ and $z^{1}-z^{0}$, as indicated in Figure 4. The distance

$$
I=\left\|z^{2}-z^{1}\right\|^{2}=\left(x^{2}-x^{1}\right)^{2}+\left(u^{2}-u^{1}\right)^{2}
$$

is not on the list and so, by Theorem 2.5, must be a function of the fundamental invariants. To find the function, we invariantize $I=\iota(I)$ by replacing each variable by its invariantized counterpart, so

$$
\begin{aligned}
\left\|z^{2}-z^{1}\right\|^{2}=I=\iota(I) & =\left\|z^{2}-z^{0}\right\|^{2} \sin ^{2} \phi+\left(\left\|z^{2}-z^{0}\right\| \cos \phi-\left\|z^{1}-z^{0}\right\|\right)^{2} \\
& =\left\|z^{1}-z^{0}\right\|^{2}+\left\|z^{2}-z^{0}\right\|^{2}-2 \cos \phi\left\|z^{1}-z^{0}\right\|\left\|z^{2}-z^{0}\right\|,
\end{aligned}
$$


thereby producing a "simple" moving frame proof of the Law of Cosines!

Example 3.9. As a final example, consider the projective action of $\operatorname{PSL}(m+1, \mathbb{R})$ on $m$-dimensional projective space $^{\dagger} M=\mathbb{R P}^{m}$. The action is given in projective coordinates

$$
z \longmapsto w=\frac{A z+b}{c \cdot z+d}, \quad \text { where } \quad\left(\begin{array}{cc}
A & b \\
c^{T} & d
\end{array}\right) \in \mathrm{GL}(m+1, \mathbb{R}), \quad z \in \mathbb{R}^{m}
$$

Here $A$ is an $m \times m$ matrix, $b, c$ are vectors and $d$ is a scalar. The point $z$ lies in the open subset $\mathbb{R}^{m} \subset \mathbb{R P}^{m}$; the remainder $\mathbb{R P}^{m} \backslash \mathbb{R}^{m} \simeq \mathbb{R P}^{m-1}$ consists of the projective directions "at $\infty$ ". We require $n \geq m+2$ in order that the action be free on an open subset of $M^{\times(n+1)}$. We begin by normalizing $w^{0}=0$, which requires $b=-A z^{0}$. The most convenient normalizations ${ }^{\ddagger}$ are to send the next $m$ points $w^{1}, \ldots, w^{m}$ off to $\infty$ along the coordinate directions, which we write as $w^{k}=\infty e_{k}$. This requires that

$$
A\left(z^{k}-z^{0}\right)=\lambda_{k} e_{k}, \quad c \cdot z^{k}+d=0, \quad k=1, \ldots, m,
$$

where the $\lambda_{k}$ 's are unspecified scalars. The solution to this linear system is given by

$$
A=\Lambda Z^{-1}, \quad c \cdot z^{k}+d=\mu V(1,2, \ldots, m, k), \quad k=1,2, \ldots,
$$

where $\mu \in \mathbb{R}$ is a scalar, $\Lambda=\operatorname{diag}\left(\lambda_{1}, \ldots, \lambda_{m}\right)$ is a diagonal matrix, and the matrix $Z$ is as in (3.5). Note that the normalization (3.16) implies that the individual entries of $v^{k}=A\left(z^{k}-z^{0}\right)$ are given by

$$
v_{i}^{k}=\left[A\left(z^{k}-z^{0}\right)\right]_{i}=m ! \lambda_{i} \frac{V(0,1, \ldots, i-1, k, i+1, \ldots, m)}{V(0,1, \ldots, m)}, \quad \begin{aligned}
& i=1, \ldots, m, \\
& k=0,1,2, \ldots
\end{aligned}
$$

Substituting these formulae back into (3.15), we find that the resulting components of

$$
w^{m+1}=\frac{A\left(z^{m+1}-z^{0}\right)}{c \cdot\left(z^{m+1}-z^{1}\right)}
$$

are

$$
w_{i}^{m+1}= \pm \frac{\lambda_{i}}{\mu} \frac{V(0, \ldots, i-1, i+1, \ldots, m+1)}{V(0,1, \ldots, m) V(1,2, \ldots, m+1)}, \quad i=1, \ldots, m .
$$

We can thus normalize $w^{m+1}=(1,1, \ldots, 1)$ by setting

$$
\lambda_{i}=\frac{\mu V(0,1, \ldots, m) V(1,2, \ldots, m+1)}{V(0, \ldots, i-1, i+1, \ldots, m+1)}, \quad k=1, \ldots, m .
$$

The last remaining group parameter $\mu$ cannot be normalized, indicative of the fact that $\mathrm{GL}(m+1)$ does not act effectively on projective space. (One can prescribe the value of $\mu$ by requiring that the matrix $\left(\begin{array}{cc}A & b \\ c^{T} & d\end{array}\right)$ be unimodular, but this is unnecessary.) Substituting

$\dagger$ The same computations apply to the complex action of $\operatorname{SL}(m+1, \mathbb{C})$ on $M=\mathbb{C P}^{m}$.

$\ddagger$ However, see Example 8.9 below. 


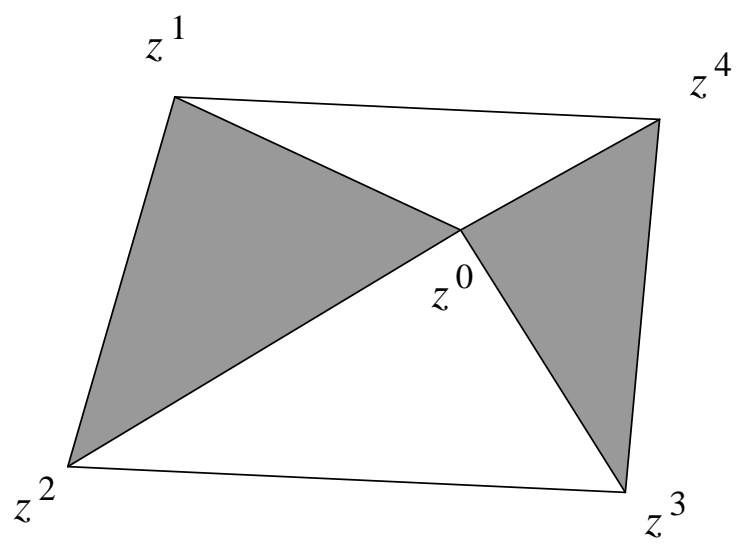

Figure 5. Projective Area Cross Ratio Invariant.

the full moving frame formulae (3.17), (3.19) into $w^{k}$ for $k \geq m+2$, we discover that the fundamental $m$-dimensional projective joint invariants are the volume cross-ratios

$$
C\left(i_{0}, \ldots, i_{m-2} ; j, k, l, n\right)=\frac{V\left(i_{0}, \ldots, i_{m-2}, j, k\right) V\left(i_{0}, \ldots, i_{m-2}, l, n\right)}{V\left(i_{0}, \ldots, i_{m-2}, j, l\right) V\left(i_{0}, \ldots, i_{m-2}, k, n\right)} .
$$

We have therefore produced a Geometric First Main Theorem for the Projective Group, a case only briefly mentioned in Weyl, [43; pp. 112-114]. Earlier, Veblen and Young, [42; $\S 27]$, derived the volume cross-ratios as basic projective invariants, but did not prove their completeness, nor did they discuss minimal generating sets.

Theorem 3.10. Every joint invariant of the projective group $\operatorname{PSL}(m+1)$ is a function of the fundamental volume cross-ratios

$$
C(0,1, \ldots, i-1, i+1, \ldots, m-1 ; i, m, m+1, k), \quad \begin{array}{ll}
i & =0, \ldots, m-1, \\
& k=m+2, m+3, \ldots
\end{array}
$$

In the one-dimensional case, these joint invariants reduce to the ordinary cross-ratios:

$$
C(0,1,2,3)=\frac{\left(z^{1}-z^{0}\right)\left(z^{3}-z^{2}\right)}{\left(z^{1}-z^{2}\right)\left(z^{3}-z^{0}\right)} .
$$

Thus, Theorem 3.10 reduces to the classical result, cf. [32], that every joint invariant of the projective group $\operatorname{PSL}(2)$ on $\mathbb{R P}^{1}$ is a function of the cross-ratios $C(0,1,2, k)$ for $k=3,4, \ldots$. In the two-dimensional case, the five-point joint invariants are generated by the following two ratios of triangular areas

$$
C(0 ; 1,2,3,4)=\frac{V(0,1,2) V(0,3,4)}{V(0,1,3) V(0,2,4)}, \quad C(1 ; 0,2,3,4)=\frac{V(0,1,2) V(1,3,4)}{V(0,1,3) V(1,2,4)}
$$

For example, $\mathrm{C}(0 ; 1,2,3,4)$ equals the product of the areas of the two shaded triangles divided by the product of the areas of the two white triangles in Figure 5. All other five-point area cross-ratios can be obtained as functions of these two. 
Similarly, in the three-dimensional case, there are 3 fundamental 6-point simplex (tetrahedron) volume cross-ratios:

$$
C(0,1 ; 2,3,4,5), \quad C(0,2 ; 1,3,4,5), \quad C(1,2 ; 0,3,4,5) .
$$

Geometrically, $C(0,1 ; 2,3,4,5)$ is obtained as a ratio of the volumes of the four simplices in the union of two pyramids, having a common quadrilateral base with vertices $z^{2}, z^{3}, z^{4}, z^{5}$, and respective apices ${ }^{\dagger}$ (apexes) $z^{0}, z^{1}$. Joining the apices by an additional line segment decomposes the double pyramid into the required four simplices. Figure 5 can be reinterpreted as a bird's eye view of this pyramidal configuration. Again, all additional 6-point volume cross-ratios are suitable functional combinations of these three. The explicit syzygies can be effectively constructed using invariantization.

These examples serve to illustrate some of the power of the normalization approach to moving frames for producing complete classifications of joint invariants for very general Lie group actions. We now turn to the applications to differential invariants.

\section{Prolongation and Differential Invariants.}

Whenever a group acts on functions, there is an induced action on their derivatives, known as "prolongation". The prolonged group transformations act on the jet bundle over the original manifold, cf. $[\mathbf{3 1}, \mathbf{3 2}]$. Jet space is the natural setting for the traditional moving frames in geometry, for the Lie theory of symmetry groups of differential equations, and for describing differential invariants. A sufficiently high order prolongation will make the group action (locally) free, and hence a moving frame can be constructed on a suitably high order jet bundle. In this section, we briefly review the basic ideas - as they apply to general parametrized submanifolds - and then show how the moving frame construction can be employed to produce complete classifications of differential invariants.

Let $\mathrm{J}^{n}=\mathrm{J}^{n}(M, p)$ denote the $n^{\text {th }}$ order (extended) jet bundle consisting of equivalence classes of $p$-dimensional submanifolds $S \subset M$ under the equivalence relation of $n^{\text {th }}$ order contact, cf. [31; Chapter 3]. We let $\mathrm{j}_{n} S \subset \mathrm{J}^{n}$ denote the $n$-jet of the submanifold $S$. We introduce local coordinates $z=(x, u)$ on $M$, considering the first $p$ components $x=\left(x_{1}, \ldots, x_{p}\right)$ as independent variables, and the latter $q=m-p$ components $u=\left(u^{1}, \ldots, u^{q}\right)$ as dependent variables. The induced local coordinates on $\mathrm{J}^{n}$ are denoted by $z^{(n)}=\left(x, u^{(n)}\right)$, with components $u_{J}^{\alpha}=D_{J} u^{\alpha}$ representing the partial derivatives of the dependent variables with respect to the independent variables. We use the notation $D_{J}=D_{x_{j_{1}}} \cdots D_{x_{j_{k}}}, 1 \leq j_{\nu} \leq p$, for the usual total derivative operators of order $k=\# J$.

For a $p$-dimensional submanifold $S \subset M$ parametrized by $z(t)=(x(t), u(t))$, where $t=\left(t_{1}, \ldots, t_{p}\right) \in T \subset \mathbb{R}^{p}$, the jet coordinates of $\mathrm{j}_{n} S$ are obtained by implicit differentiation. If we write the "horizontal" one-forms $d x=\left(d x_{1}, \ldots, d x_{p}\right)^{T}$ as components of a column vector, then the chain rule implies

$$
d x=D_{t} x \cdot d t
$$

\footnotetext{
$\dagger$ It is not often one gets to use the plurals of "vertex", "simplex", and "apex" in the same sentence!
} 
where $D_{t} x=\left(D_{t_{i}} x_{j}\right)$ denotes the $p \times p$ Jacobian matrix of $x(t)$. The dual total differential operators $D_{x}=\left(D_{x_{1}}, \ldots, D_{x_{p}}\right)^{T}$ are then given by

$$
D_{x}=\left(D_{t} x\right)^{-T} D_{t}
$$

One recursively applies these operators to the dependent variables $u^{\alpha}$ to obtain the jet coordinates $u_{J}^{\alpha}=D_{J} u^{\alpha}$ of a general parametrized submanifold. The resulting formulae are clearly independent of the parametrization.

Let $G$ be a Lie group acting smoothly on $M$; in terms of local coordinates $z=(x, u)$ we write the group transformations $w=(y, v)=g \cdot z$ as

$$
w=(y(g, z), v(g, z))=(y(g, x, u), v(g, x, u)) .
$$

Given a submanifold $S$ parametrized by $z(t)=(x(t), u(t))$, the transformed submanifold $\bar{S}=g \cdot S$ is parametrized by

$$
w(t)=g \cdot z(t)=(y(g, z(t)), v(g, z(t))) .
$$

Since smooth transformations preserve the order of contact between submanifolds, there is an induced action, denoted by $G^{(n)}$, on the $n^{\text {th }}$ order jet bundle $\mathrm{J}^{n}$, known as the $n^{\text {th }}$ prolongation of the group action. Explicit formulae for the prolonged group transformations

$$
w^{(n)}=\left(y, v^{(n)}\right)=g^{(n)} \cdot\left(x, u^{(n)}\right)
$$

are found by implicit differentiation of the transformed dependent variables $v(g, z)$ with respect to the transformed independent variables $y(g, z)$. We use the chain rule to determine the horizontal one-forms

$$
d y=D_{t} y \cdot d t=\left[D_{z} y \cdot D_{t} z\right] d t
$$

and thereby obtain the associated total derivative operators

$$
D_{y}=\left(D_{t} y\right)^{-T} D_{t}=\left[D_{z} y \cdot D_{t} z\right]^{-T} D_{t} \text {. }
$$

As before, the resulting prolongation formulae

$$
v_{J}^{\alpha}=D_{y_{j_{1}}} \cdots D_{y_{j_{k}}} v^{\alpha}
$$

for the coordinates of $w^{(n)}=g^{(n)} \cdot z^{(n)}$ are independent of parametrization.

Example 4.1. The planar Euclidean group $\mathrm{SE}(2)$ acts on $M=\mathbb{R}^{2}$, mapping a point $z=(x, u)$ to

$$
y=x \cos \theta-u \sin \theta+a, \quad v=x \sin \theta+u \cos \theta+b .
$$

For a parametrized curve, $z(t)=(x(t), u(t))$, we have

$$
d x=x_{t} d t, \quad \text { and so } \quad D_{x}=\frac{1}{x_{t}} D_{t} .
$$


The parametric curve's second order jet coordinates are then given by

$$
\left(x, u, u_{x}, u_{x x}\right)=\left(x, u, \frac{u_{t}}{x_{t}}, \frac{x_{t} u_{t t}-u_{t} x_{t t}}{x_{t}^{3}}\right),
$$

Similarly, for the transformed curve $w(t)=(y(t), v(t))$, we have

$$
d y=\left(x_{t} \cos \theta-u_{t} \sin \theta\right) d t
$$

and so the implicit differentiation operator (4.5) is given by

$$
D_{y}=\frac{1}{x_{t} \cos \theta-u_{t} \sin \theta} D_{t}
$$

Therefore, a Euclidean transformation $g \in \mathrm{SE}(2)$ has second order prolongation that maps the jet $(4.9)$ to $\left(y, v, v_{y}, v_{y y}\right)=g^{(2)} \cdot\left(x, u, u_{x}, u_{x x}\right)$, where $y, v$ are given by $(4.7)$ and

$$
v_{y}=\frac{d v}{d y}=\frac{x_{t} \sin \theta+u_{t} \cos \theta}{x_{t} \cos \theta-u_{t} \sin \theta}, \quad v_{y y}=\frac{d^{2} v}{d y^{2}}=\frac{x_{t} u_{t t}-x_{t t} u_{t}}{\left(x_{t} \cos \theta-u_{t} \sin \theta\right)^{3}} .
$$

These formulae, of course, depend only on the jets (4.9) and not the particular parametrization.

An $n^{\text {th }}$ order moving frame $\rho^{(n)}: \mathrm{J}^{n} \rightarrow G$ is an equivariant map defined on an open subset of the jet space. Application of our moving frame construction requires freeness and regularity of the prolonged group action. At present, the most general result in this direction is based on a theorem of Ovsiannikov, cf. [35, 32]; additional details, including a correct extension of this result to smooth actions, can be found in $[\mathbf{3 4}]$.

Theorem 4.2. Let $G$ be an analytic transformation group acting locally effectively on $M$. For $n \gg 0$ sufficiently large, the $n^{\text {th }}$ order prolongation $G^{(n)}$ acts locally freely on a dense open subset $\mathcal{V}^{n} \subset \mathrm{J}^{n}(M, p)$.

An outstanding conjecture is that, in the analytic category, $G^{(n)}$ actually acts freely and regularly on an open subset of a sufficiently high order jet bundle $\mathrm{J}^{n}$. Unfortunately, the proof of Theorem 4.2 is based on infinitesimal methods and so cannot prove this more general result. Nevertheless, freeness does happen in all known examples.

Definition 4.3. A jet $z^{(n)} \in \mathrm{J}^{n}$ is called regular if $G$ acts (locally) freely in an neighborhood $z^{(n)} \in U \subset \mathrm{J}^{n}$ thereof.

Theorem 4.2 implies that almost all jets of sufficiently high order are regular. The next result is an immediate consequence of Theorem 2.2.

Theorem 4.4. If $G$ acts analytically and effectively, then an $n^{\text {th }}$ order moving frame exists in a neighborhood of a point $z^{(n)} \in \mathrm{J}^{n}$ if and only if $z^{(n)} \in \mathcal{V}^{n}$ is a regular jet. 
We can now apply our normalization construction to produce a moving frame and a complete system of differential invariants in the neighborhood of any regular jet. We begin by using implicit differentiation (4.6) to compute the prolonged transformation formulae

$$
w^{(n)}\left(g, z^{(n)}\right)=g^{(n)} \cdot z^{(n)} .
$$

For simplicity, we restrict to a coordinate cross-section

$$
K=\left\{z_{1}=c_{1}, \ldots, z_{r}=c_{r}\right\} \subset \mathrm{J}^{n},
$$

choosing $r=\operatorname{dim} G$ components $w_{1}, \ldots, w_{r}$ of $w^{(n)}$ to normalize to constants. Solving the associated normalization equations

$$
w_{1}\left(g, z^{(n)}\right)=c_{1}, \quad \ldots \quad w_{r}\left(g, z^{(n)}\right)=c_{r},
$$

for the group transformations leads to the explicit formulae

$$
g=\rho^{(n)}\left(z^{(n)}\right)
$$

for the right moving frame $\rho^{(n)}: \mathrm{J}^{n} \rightarrow G$. In classical situations, the left counterpart $\widetilde{\rho}^{(n)}\left(z^{(n)}\right)=\rho^{(n)}\left(z^{(n)}\right)^{-1}$, when restricted to the submanifold $\mathrm{j}_{n} S$, can be identified with the usual geometric moving frame, cf. $[\mathbf{2 1}, \mathbf{2 2}, \mathbf{2 4}]$.

Remark: Composing an $n^{\text {th }}$ order moving frame $\rho^{(n)}: \mathrm{J}^{n} \rightarrow G$ with the standard jet bundle projection $\pi_{n}^{k}: \mathrm{J}^{k} \rightarrow \mathrm{J}^{n}$ for any $k \geq n$ automatically produces a moving frame $\rho^{(k)}=\rho^{(n)} \circ \pi_{n}^{k}$ on (an open subset of) the jet bundle $\mathrm{J}^{k}$. In this manner, moving frames are immediately applied to compute all higher order differential invariants.

Definition 4.5. A differential invariant is an invariant function for a prolonged transformation group.

According to Theorem 2.5, substituting the moving frame formulae (4.15) into the unnormalized components of $w^{(n)}$, or, more generally, $w^{(k)}$ for any $k \geq n$, leads to a complete system of $k^{\text {th }}$ order differential invariants.

Definition 4.6. The fundamental $n^{\text {th }}$ order differential invariants associated with a moving frame $\rho^{(n)}$ of order $n$ (or less) are given by

$$
I^{(n)}\left(z^{(n)}\right)=w^{(n)}\left(\rho^{(n)}\left(z^{(n)}\right), z^{(n)}\right)=\rho^{(n)}\left(z^{(n)}\right) \cdot z^{(n)} .
$$

In terms of the local coordinates $w^{(n)}=\left(y, v^{(n)}\right)$, the fundamental differential invariants will be denoted by

$$
\begin{aligned}
& y_{i} \longmapsto H_{i}\left(x, u^{(n)}\right)=y_{i}\left(\rho^{(n)}\left(x, u^{(n)}\right), x, u\right), \quad i=1, \ldots, p, \\
& v_{K}^{\alpha} \longmapsto I_{K}^{\alpha}\left(x, u^{(k)}\right)=v_{K}^{\alpha}\left(\rho^{(n)}\left(x, u^{(n)}\right), x, u^{(k)}\right), \quad \alpha=1, \ldots, q, \quad k=\# K \geq 0 .
\end{aligned}
$$

In particular, the fundamental differential invariants corresponding to the normalization components (4.14) of $w^{(n)}$ will be constant, and are known as the phantom differential invariants. According to Theorem 2.5, the other components of $w^{(n)}$ will define a complete system of functionally independent differential invariants defined on the domain of definition of the moving frame map. 
Theorem 4.7. Let $\rho^{(n)}: \mathrm{J}^{n} \rightarrow G$ be a moving frame of order $\leq n$. Every $n^{\text {th }}$ order differential invariant can be locally written as a function $J=\Phi\left(I^{(n)}\right)$ of the fundamental $n^{\text {th }}$ order differential invariants. The function $\Phi$ is unique provided it does not depend on the phantom invariants.

The invariantization of a differential function $F: \mathrm{J}^{n} \rightarrow \mathbb{R}$ with respect to the given moving frame is the differential invariant $J=\iota(F)=F \circ I^{(n)}$. As before, invariantization defines a projection, depending on the moving frame, from the space of differential functions to the space of differential invariants.

Example 4.8. The classical moving frame, $[\mathbf{2 4}]$, for curves under the planar Euclidean group consists of the point $z=(x, u)^{T}$ on the curve along with the unit tangent and unit normal vectors

$$
\mathbf{t}=\frac{z_{t}}{\left\|z_{t}\right\|}=\frac{1}{\sqrt{x_{t}^{2}+u_{t}^{2}}}\left(\begin{array}{c}
x_{t} \\
u_{t}
\end{array}\right), \quad \mathbf{n}=\mathbf{t}^{\perp}=\frac{1}{\sqrt{x_{t}^{2}+u_{t}^{2}}}\left(\begin{array}{c}
-u_{t} \\
x_{t}
\end{array}\right) .
$$

These provide a left moving frame $\rho: \mathrm{J}^{1} \rightarrow \mathrm{SE}(2)$ in the sense of Definition 2.1 when we identify $\widetilde{d}=-R^{T} d=z$ as the translation component and $\widetilde{R}=R^{T}=(\mathbf{t} \mathbf{n})$ as the rotation component of $\widetilde{g}=\widetilde{\rho}^{(1)}\left(z^{(1)}\right)$. If we substitute the moving frame normalizations (4.18) into the prolonged transformation formulae for, say, the second, third and fourth derivatives, cf. (4.11), we obtain the fundamental normalized differential invariants

$$
v_{y y} \longmapsto \kappa, \quad v_{y y y} \longmapsto \frac{d \kappa}{d s}, \quad v_{y y y y} \longmapsto \frac{d^{2} \kappa}{d s^{2}}+3 \kappa^{3},
$$

where

$$
\kappa=\frac{z_{t} \wedge z_{t t}}{\left\|z_{t}\right\|^{3}}=\frac{x_{t} u_{t t}-x_{t t} u_{t}}{\left(x_{t}^{2}+u_{t}^{2}\right)^{3 / 2}} .
$$

is the Euclidean curvature invariant, and

$$
D_{s}=\frac{1}{\sqrt{x_{t}^{2}+u_{t}^{2}}} D_{t}=\frac{1}{\left\|z_{t}\right\|} D_{t}
$$

denotes differentiation with respect to the Euclidean arc length element, which may also be obtained by normalization:

$$
d y=\left(x_{t} \cos \theta+u_{t} \sin \theta\right) d t \quad \longmapsto \quad d s=\sqrt{x_{t}^{2}+u_{t}^{2}} d t=\left\|z_{t}\right\| d t .
$$

(See the discussion in the following section.) A complete system of differential invariants for the planar Euclidean group is provided by the curvature and its successive derivatives with respect to arc length: $\kappa, \kappa_{s}, \kappa_{s s}, \ldots$.

There are, however, some interesting subtleties in this standard construction which can now be appreciated. First, the classical moving frame (4.18) is only equivariant for oriented curves under the orientation-preserving Euclidean group SE(2). Indeed, reversing the parametrization, $t \mapsto-t$, reverses the direction of the tangent vector $\mathbf{t}$, while applying a reflection changes the direction of $\mathbf{n}$. Moreover, the Euclidean curvature $\kappa$ changes sign under either operation, while the sign of the arc length element $d s$ changes under reversal 
of parametrization. Thus, while all of these quantities are intrinsic to oriented curves (e.g., closed curves parametrized in a counterclockwise direction) in the oriented Euclidean plane, they all have sign ambiguities in the more general setting considered here.

Let us see how these ambiguities can be resolved in this simple example. We write the Euclidean group element as

$$
z \longmapsto \widetilde{R}^{T}(z-\widetilde{d}), \quad \text { where } \quad \widetilde{R}=(\mathbf{a} \mathbf{b})
$$

belongs to either $\mathrm{SO}(2)$ or $\mathrm{O}(2)$ with orthonormal frame vectors $\mathbf{a}, \mathbf{b}$. The classical moving frame is based on the simple coordinate cross-section

$$
K^{(1)}=\left\{x=u=u_{x}=0\right\} .
$$

However, the action (4.7), (4.11) is only locally free on $\mathrm{J}^{1}$ and requires prolonging further to become free and thereby produce a legitimate cross-section. Ignoring this detail for a moment, the associated normalization equations

$$
y=\mathbf{a} \cdot(z-\widetilde{d})=0, \quad v=\mathbf{b} \cdot(z-\widetilde{d})=0, \quad v_{y}=\frac{\mathbf{b} \cdot z_{t}}{\mathbf{a} \cdot z_{t}}=0,
$$

have the general solution

$$
\mathbf{a}=\varepsilon \mathbf{t}=\varepsilon \frac{z_{t}}{\left\|z_{t}\right\|}, \quad \mathbf{b}=\sigma \varepsilon \mathbf{n}=\sigma \varepsilon \mathbf{t}^{\perp}, \quad \widetilde{d}=z .
$$

where $\sigma, \varepsilon= \pm 1$ with $\sigma=1$ when $G=\mathrm{SE}(2)$. In the case of an oriented curve, the direction of $\mathbf{t}$ is fixed, and so $\varepsilon=1$ is specified, while the restriction to the proper Euclidean group $\operatorname{SE}(2)$ specifies $\sigma=+1$, and hence we recover the classical moving frame (4.18). However, in general there remain 1 or 2 sign ambiguities to resolve, stemming from the local but not global freeness of the first order prolongation.

To resolve the sign ambiguities, we must consider the action on higher order derivatives. As before, the arc length form is obtained by normalizing

$$
d y=\left(\mathbf{a} \cdot z_{t}\right) d t \quad \longmapsto \quad \varepsilon d s=\varepsilon\left\|z_{t}\right\| d t .
$$

The second order derivative becomes

$$
v_{y y}=\frac{\left(\mathbf{a} \cdot z_{t}\right)\left(\mathbf{b} \cdot z_{t t}\right)-\left(\mathbf{a} \cdot z_{t t}\right)\left(\mathbf{b} \cdot z_{t}\right)}{\left(\mathbf{a} \cdot z_{t}\right)^{3}}=\frac{\sigma z_{t} \wedge z_{t t}}{\left(\mathbf{a} \cdot z_{t}\right)^{3}} \longmapsto \sigma \varepsilon \kappa,
$$

where $\kappa$ is the signed Euclidean curvature (4.20). Therefore, provided we are not at an inflection point ${ }^{\dagger}$ where $\kappa=0$, we can specify

$$
\sigma \varepsilon=\operatorname{sgn} \kappa,
$$

producing the differential invariant $|\kappa|$. The third order derivative reduces to

$$
v_{y y y} \longmapsto \sigma \frac{\left\|z_{t}\right\|^{2}\left(z_{t} \wedge z_{t t t}\right)-\left(z_{t} \cdot z_{t t}\right)\left(z_{t} \wedge z_{t t}\right)}{\left\|z_{t}\right\|^{5}}=\sigma \kappa_{s} .
$$

$\dagger$ A moving frame, of higher order, can be constructed near nondegenerate inflection points. 
Therefore, in the case of $\mathrm{E}(2)$, one can set $\sigma=\operatorname{sgn} \kappa_{s}$.

Summarizing, in the oriented case of $\mathrm{SE}(2)$, freeness requires a second order crosssection

$$
K^{(2)}=\left\{x=u=u_{x}=0, u_{x x}>0\right\},
$$

and the corresponding left moving frame is given by

$$
\widetilde{R}=(\operatorname{sgn} \kappa)(\mathbf{t} \mathbf{n}), \quad \widetilde{d}=z .
$$

The fundamental differential invariant and invariant one form are

$$
|\kappa|, \quad(\operatorname{sgn} \kappa) d s=(\operatorname{sgn} \kappa)\left\|z_{t}\right\| d t .
$$

All higher order differential invariants, $\kappa_{s},(\operatorname{sgn} \kappa) \kappa_{s s}$, etc., are obtained via invariant differentiation. In the non-oriented case of $\mathrm{E}(2)$, we require a third order cross-section

$$
K^{(3)}=\left\{x=u=u_{x}=0, u_{x x}>0, u_{x x x}>0\right\},
$$

and the corresponding left moving frame is

$$
\widetilde{R}=\left(\left(\operatorname{sgn} \kappa_{s}\right) \mathbf{t},(\operatorname{sgn} \kappa) \mathbf{n}\right), \quad \widetilde{d}=z .
$$

The fundamental differential invariant and invariant one form are

$$
|\kappa|, \quad \operatorname{sgn}\left(\kappa \kappa_{s}\right) d s=\operatorname{sgn}\left(\kappa \kappa_{s}\right)\left\|z_{t}\right\| d t .
$$

All higher order differential invariants, $\left|\kappa_{s}\right|, \operatorname{sgn}(\kappa) \kappa_{s s}$ and so on, are found by invariant differentiation.

\section{Recurrence Formulae and Syzygies.}

In general, higher order differential invariants can all be obtained by invariantly differentiating certain fundamental differential invariants. On the other hand, one can obtain higher order differential invariants directly by normalizing using the moving frame, the result being the fundamental differential invariants (4.17). The fact that the normalized higher order invariants do not, in general, agree with the differentiated invariants is of crucial significance. A general algorithm, first found in $[\mathbf{1 9}, \mathbf{2 0}]$, for determining the "correction terms" - for example, the $3 \kappa^{3}$ in the last formula in (4.19) — will now be presented.

Each moving frame $\rho^{(n)}: \mathrm{J}^{n} \rightarrow G$ produces an associated collection of invariant differential operators, which are obtained by applying the normalization formulae to the implicit differential operators (4.5). First, normalizing the one-forms (4.4),

$$
d y=D_{t} y\left(g, z^{(1)}\right) d t \longmapsto \omega=D_{t} y\left(\rho^{(n)}\left(z^{(n)}\right), z^{(1)}\right) d t \equiv P\left(z^{(n)}\right) d t,
$$

leads to a system of $p$ linearly independent invariant one-forms $\omega=\left(\omega_{1}, \ldots, \omega_{p}\right)^{T}$, known as an invariant horizontal coframe of order $n$. The associated invariant differential operators $\mathcal{D}_{1}, \ldots, \mathcal{D}_{p}$ are uniquely defined so that

$$
d F=\sum_{j=1}^{p}\left(\mathcal{D}_{i} F\right) \omega_{i}, \quad \text { for any } \quad F: \mathrm{J}^{n} \rightarrow \mathbb{R} .
$$


They may be obtained directly by substituting the normalization formulae $g=\rho^{(n)}\left(z^{(n)}\right)$ into the implicit differentiation operators (4.5):

$$
D_{y}=D_{t} y\left(g, z^{(1)}\right)^{-T} D_{t} \longmapsto \mathcal{D}=D_{t} y\left(\rho^{(n)}\left(z^{(n)}\right), z^{(1)}\right)^{-T} D_{t}=P\left(z^{(n)}\right)^{-T} D_{t} .
$$

The invariant differential operators (5.3) will map differential invariants to higher order differential invariants.

Theorem 5.1. Let $\rho^{(n)}: \mathcal{V}^{n} \rightarrow G$ be an $n^{\text {th }}$ order moving frame defined on an open subset $\mathcal{V}^{n} \subset \mathrm{J}^{n}$. Then for any $k \geq n+1$, a complete system of $k^{\text {th }}$ order differential invariants on $\mathcal{V}^{k}=\left(\pi_{n}^{k}\right)^{-1} \mathcal{V}^{n} \subset \mathrm{J}^{k}$ can be found by successively applying the invariant differential operators $\mathcal{D}_{1}, \ldots, \mathcal{D}_{p}$ to the non-constant (non-phantom) components of the fundamental differential invariants $I^{(n+1)}$ of order at most $n+1$.

As we noted in (4.19), the differentiated invariants do not in general coincide with the normalized differential invariants. The fundamental recurrence formulae

$$
\mathcal{D}_{j} H_{i}=\delta_{j}^{i}-L_{j}^{i}, \quad \mathcal{D}_{j} I_{K}^{\alpha}=I_{K, j}^{\alpha}-M_{K, j}^{\alpha},
$$

for the differentiated invariants (4.17) are of critical importance in practical applications. First, they permit one to straightforwardly produce a minimal generating system of differential invariants, with the property that every other differential invariant is obtained by invariant differentiation. Although Theorem 5.1 requires all of the fundamental invariants of order $\leq n+1$ to generate the higher order differential invariants, one typically can also generate a number of the components of $I^{(n+1)}$ in this manner, and so a minimal system of generating differential invariants is some subset of the non-phantom differential invariants of order $\leq n+1$. Furthermore, the syzygies or functional relationships among the differentiated invariants can be systematically determined by analysis of the recurrence formulae (5.4); see $[\mathbf{1 9}, \mathbf{2 0}]$ for details. Finally, the recurrence formulae are basic to the detailed analysis of the differential invariant and joint invariant signatures resulting from the moving frame construction, as we shall see in the examples below.

Remark: In Weyl's algebraic formulation of the "Second Main Theorem" for the group action, [43], syzygies are defined as algebraic relations among the joint invariants. Here, since we are classifying invariants up to functional independence, there are no algebraic syzygies. Thus, the classification of differential syzygies is the proper setting for the Second Main Theorem in the geometric/analytic context.

Fortunately, the correction terms $L_{j}^{i}, M_{K, j}^{\alpha}$ in the recurrence formulae (5.4) can be effectively computed using the following infinitesimal algorithm, first presented in [20]. The remarkable fact is that they can be found - and hence complete classifications of generating systems of differential invariants and syzygies can be established - without knowledge of the explicit formulae for the normalized differential invariants! All one needs is the normalization constants (4.14) and the well-known prolongation formulae for the infinitesimal generators of the transformation group, cf. [31, 32].

Choose a basis $\left\{\mathbf{v}_{1}, \ldots, \mathbf{v}_{r}\right\}$ for the Lie algebra $\mathfrak{g}$ of infinitesimal generators of the group action on $M$. Let $\left\{\mathrm{pr}^{(n)} \mathbf{v}_{1}, \ldots, \mathrm{pr}^{(n)} \mathbf{v}_{r}\right\}$ denote the corresponding basis for the Lie 
algebra $\mathfrak{g}^{(n)}$ of infinitesimal generators of the prolonged group action $G^{(n)}$. The prolonged generators are obtained by truncating, at order $n$, the infinitely prolonged vector fields

$$
\begin{gathered}
\operatorname{pr} \mathbf{v}_{\kappa}=\sum_{i=1}^{p} \xi_{\kappa}^{i}(x, u) \frac{\partial}{\partial x^{i}}+\sum_{\alpha=1}^{q} \sum_{k=\# J \geq 0} \varphi_{J, \kappa}^{\alpha}\left(x, u^{(k)}\right) \frac{\partial}{\partial u_{J}^{\alpha}}, \quad \text { where } \\
\varphi_{J, \kappa}^{\alpha}=D_{J} Q_{\kappa}^{\alpha}+\sum_{i=1}^{p} \xi_{\kappa}^{i} u_{J, i}^{\alpha}, \quad Q_{\kappa}^{\alpha}\left(x, u^{(1)}\right)=\varphi_{\kappa}^{\alpha}(x, u)-\sum_{i=1}^{p} \xi_{\kappa}^{i}(x, u) u_{i}^{\alpha} .
\end{gathered}
$$

Here $Q_{\kappa}=\left(Q_{\kappa}^{1}, \ldots, Q_{\kappa}^{q}\right)$ is the characteristic of $\mathbf{v}_{\kappa}$, and $D_{J}$ denotes total differentiation.

Definition 5.2. The Lie matrix of order $n$ is defined as the $r \times\left[p+q\left(\begin{array}{c}p+n \\ n\end{array}\right)\right]$ matrix

$$
\mathbf{L}_{n}\left(z^{(n)}\right)=\left(\begin{array}{ccccccccc}
\xi_{1}^{1} & \ldots & \xi_{1}^{p} & \varphi_{1}^{1} & \ldots & \varphi_{1}^{q} & \ldots & \varphi_{J, 1}^{\alpha} & \ldots \\
\vdots & \ddots & \vdots & \vdots & \ddots & \vdots & \ddots & \vdots & \ddots \\
\xi_{r}^{1} & \ldots & \xi_{r}^{p} & \varphi_{r}^{1} & \ldots & \varphi_{r}^{q} & \ldots & \varphi_{J, r}^{\alpha} & \ldots
\end{array}\right)
$$

whose entries $\xi_{\kappa}^{i}$, and $\varphi_{J, \kappa}^{\alpha}, 0 \leq \# J \leq n$, are the coefficients of the $n^{\text {th }}$ order prolongations (5.5) of the basis infinitesimal generators of $G$.

Remark: Note that $\operatorname{rank} \mathbf{L}_{n}\left(z^{(n)}\right)$ equals the dimension of the subspace of the tangent space $\left.T \mathrm{~J}^{n}\right|_{z^{(n)}}$ spanned by the prolonged infinitesimal generators, and hence coincides with the dimension of the orbit through $z^{(n)}$. In particular, $z^{(n)}$ is a locally regular jet if and only if $\mathbf{L}_{n}\left(z^{(n)}\right)$ has maximal rank $r=\operatorname{dim} G$.

Consider a moving frame $\rho^{(n)}: \mathrm{J}^{n} \rightarrow G$ defined (for simplicity) by a coordinate crosssection (4.14). We then form the invariantized Lie matrix

$$
\mathbf{I}_{n}=\iota\left(\mathbf{L}_{n}\right)=\mathbf{L}_{n}\left(I^{(n)}\right)
$$

which is obtained by replacing the jet coordinates $z^{(n)}=\left(x, u^{(n)}\right)$ by the normalized differential invariants $I^{(n)}$ given in (4.16). We perform a Gauss-Jordan row reduction on the matrix $\mathbf{I}_{n}$ so as to reduce the $r \times r$ minor whose columns correspond to the normalization variables $z_{1}, \ldots, z_{r}$ to an $r \times r$ identity matrix. Let $\mathbf{K}_{n}$ denote the resulting matrix of differential invariants.

Next, let $\mathbf{Z}\left(x, u^{(n)}\right)=\left(D_{i} z_{\kappa}\right)$ denote the $p \times r$ matrix whose entries are the total derivatives of the normalization coordinates $z_{1}, \ldots, z_{r}$. Let

$$
\mathbf{W}=\iota(\mathbf{Z})=\mathbf{Z}\left(I^{(n)}\right)
$$

be its invariantization. Then the correction terms in (5.4) are the entries of the matrix product

$$
\mathbf{W} \cdot \mathbf{K}_{n}=\mathbf{M}_{n}=\left(\begin{array}{ccccccccc}
L_{1}^{1} & \ldots & L_{1}^{p} & M_{1}^{1} & \ldots & M_{1}^{q} & \ldots & M_{K, 1}^{\alpha} & \ldots \\
\vdots & \ddots & \vdots & \vdots & \ddots & \vdots & \ddots & \vdots & \ddots \\
L_{r}^{1} & \ldots & L_{p}^{p} & M_{r}^{1} & \ldots & M_{r}^{q} & \ldots & M_{K, r}^{\alpha} & \ldots
\end{array}\right)
$$

where $\mathbf{K}_{n}$ is Gauss-Jordan reduced version of the invariantized Lie matrix $\mathbf{I}_{n}$. 
Note in particular that, since the non-phantom components of $I^{(n)}$ are always functionally independent, we do not require their explicit formulae in order to perform this computation. The only ingredients are the normalization coordinates $z_{1}, \ldots, z_{r}$ and constants $c_{1}, \ldots, c_{r}$ that define our chosen jet cross-section (4.13), which are the constant values attained by the phantom differential invariants.

Example 5.3. The infinitesimal generators of the planar Euclidean group SE(2) are

$$
\mathbf{v}_{1}=\partial_{x}, \quad \mathbf{v}_{2}=\partial_{u}, \quad \mathbf{v}_{3}=-u \partial_{x}+x \partial_{u} .
$$

Applying the prolongation formulae (5.5), the fifth order Lie matrix $\mathbf{L}_{5}$ equals

$$
\left(\begin{array}{ccccccc}
1 & 0 & 0 & 0 & 0 & 0 & 0 \\
0 & 1 & 0 & 0 & 0 & 0 & 0 \\
-u & x & 1+u_{x}^{2} & 3 u_{x} u_{x x} & M_{3} & M_{4} & M_{5}
\end{array}\right)
$$

where

$$
\begin{gathered}
M_{3}=4 u_{x} u_{x x x}+3 u_{x x}^{2}, \quad M_{4}=5 u_{x} u_{x x x x x}+10 u_{x x} u_{x x x}, \\
M_{5}=6 u_{x} u_{x x x x x}+15 u_{x x} u_{x x x x}+10 u_{x x x}^{2} .
\end{gathered}
$$

Under the normalizations $^{\dagger}$ (4.23), the fundamental differential invariants are

$$
y \longmapsto H=0, \quad v \longmapsto I=0, \quad v_{y} \longmapsto I_{1}=0, \quad v_{y y} \longmapsto I_{2}=\kappa,
$$

and, in general, $v_{k}=D_{y}^{k} v \longmapsto I_{k}$. The recurrence formulae will express each normalized differential invariant $I_{k}$ in terms of arc length derivatives of $\kappa=I_{2}$.

The invariantized Lie matrix takes the form

$$
\iota\left(\mathbf{L}_{5}\right)=\mathbf{I}_{5}=\left(\begin{array}{ccccccc}
1 & 0 & 0 & 0 & 0 & 0 & 0 \\
0 & 1 & 0 & 0 & 0 & 0 & 0 \\
0 & 0 & 1 & 0 & 3 \kappa^{2} & 10 \kappa I_{3} & 15 \kappa I_{4}+10\left(I_{3}\right)^{2}
\end{array}\right) .
$$

Since our chosen cross-section (4.23) is based on the jet coordinates $x, u, u_{x}$ that index the first three columns of these matrices, $\mathbf{I}_{5}$ is already in the appropriate row-reduced form. Therefore $\mathbf{K}_{5}=\mathbf{I}_{5}$. Moreover, differentiating the normalization variables and then invariantizing produces the matrices

$$
\mathbf{Z}=\left(\begin{array}{lll}
1 & u_{x} & u_{x x}
\end{array}\right), \quad \iota(\mathbf{Z})=\mathbf{W}=\left(\begin{array}{lll}
1 & 0 & I_{2}
\end{array}\right)=\left(\begin{array}{lll}
1 & 0 & \kappa
\end{array}\right) .
$$

Therefore, the fifth order correction matrix is

$$
\mathbf{M}_{5}=\mathbf{W} \cdot \mathbf{K}_{5}=\left(\begin{array}{llllllll}
1 & 0 & \kappa & 0 & 3 \kappa^{3} & 10 \kappa^{2} I_{3} & 15 \kappa^{2} I_{4}+10 \kappa\left(I_{3}\right)^{2}
\end{array}\right),
$$

whose entries are the required the correction terms. The recurrence formulae (5.4) can then be read off in order:

$$
\begin{array}{lll}
\mathcal{D} H=\mathcal{D}(0)=1-1, & \mathcal{D} I=\mathcal{D}(0)=0-0, & \mathcal{D} I_{1}=\mathcal{D}(0)=I_{2}-\kappa, \quad \mathcal{D} I_{2}=\mathcal{D} \kappa=I_{3}-0, \\
\mathcal{D} I_{3}=I_{4}-3 \kappa^{3}, & \mathcal{D} I_{4}=I_{5}-10 \kappa^{2} I_{3}, & \mathcal{D} I_{5}=I_{6}-15 \kappa^{2} I_{4}-10 \kappa\left(I_{3}\right)^{2},
\end{array}
$$

$\dagger$ For simplicity, we consider the case of oriented curves under SE(2). However, the computations apply equally well to non-oriented curves and to $\mathrm{E}(2)$. 
where $\mathcal{D}=D_{s}$ is the invariant arc length derivative (4.21). We conclude that the higher order normalized differential invariants are given in terms of arc length derivatives of the curvature $\kappa$ by

$$
\begin{array}{cl}
I_{2}=\kappa, & I_{3}=\kappa_{s}, \quad I_{4}=\kappa_{s s}+3 \kappa^{3}, \\
I_{5}=\kappa_{s s s}+19 \kappa^{2} \kappa_{s}, & I_{6}=\kappa_{s s s s}+34 \kappa^{2} \kappa_{s s}+48 \kappa \kappa_{s}^{2}+45 \kappa^{4} \kappa_{s},
\end{array}
$$

and so on. The direct derivation of these (and subsequent, more complicated) formulae is, needless to say, considerably more tedious.

\section{Equivalence and Signatures.}

Beyond the basic classification of differential invariants, the motivational application of moving frame theory is to the problems of equivalence and symmetry (i.e., self-equivalence) of submanifolds. Let $G$ be a transformation group acting on the manifold $M$. Two submanifolds $S, \bar{S} \subset M$ are said to be equivalent (or congruent) under the action of $G$ if there is a group transformation $g \in G$ mapping one to the other: $\bar{S}=g \cdot S$. A symmetry of a submanifold is a group transformation that maps $S$ to itself; we let $G_{S}=\{g \in G \mid g \cdot S=S\}$ denote the symmetry (or isotropy) subgroup of $S$. As emphasized by Cartan, [14], the solution to the equivalence and symmetry problems for submanifolds is based on the functional interrelationships among the fundamental differential invariants restricted to the submanifold, as we now review.

Recall first the Definition 4.3 of a regular jet. A submanifold is called regular of order $n$ at a point $z_{0} \in N$ if its $n^{\text {th }}$ order jet $\left.\mathrm{j}_{n} N\right|_{z_{0}}$ is regular. Any order $n$ regular submanifold admits a (locally defined) moving frame of that order - one merely restricts a moving frame defined in a neighborhood of $z_{0}$ to the submanifold: $\rho^{(n)} \circ \mathrm{j}_{n} S$. Thus, only those submanifolds having singular jets at arbitrarily high order fail to admit any moving frame whatsoever. The complete classification of such totally singular submanifolds was found in $[34]$.

Theorem 6.1. Suppose $G$ acts effectively and analytically. An analytic submanifold $S \subset M$ is totally singular if and only if its symmetry group $G_{S}$ does not act locally freely on $S$ itself.

For any $k \geq n$, the functions on a regular submanifold $S$ obtained by restricting the fundamental differential invariants,

$$
J^{(k)}=I^{(k)} \mid S=I^{(k)} \circ \mathrm{j}_{k} S,
$$

will be called the $k^{\text {th }}$ order restricted differential invariants for $S$. The $k^{\text {th }}$ order signature $\mathcal{S}^{(k)}=\mathcal{S}^{(k)}(S)$ is the set parametrized by the restricted differential invariants. The submanifold $S$ is called fully regular ${ }^{\dagger}$ if the differential invariant map (6.1) has constant rank $0 \leq t_{k} \leq p=\operatorname{dim} S$ for all $k \geq n$. Note that $t_{k}$ equals the number of functionally

$\dagger$ One can generalize these results to include submanifolds for which rank $J^{(k)}$ is constant for $k \gg 0$ sufficiently large. 
independent $k^{\text {th }}$ order restricted differential invariants. In this case, the signature $\mathcal{S}^{(k)}$ forms a submanifold of dimension $t_{k}$ - perhaps with self-intersections, although these can be resolved by further increasing the order, as discussed in [19]. In the fully regular case, the signature ranks satisfy

$$
t_{n}<t_{n+1}<t_{n+2}<\cdots<t_{s}=t_{s+1}=\cdots=t \leq p
$$

where $t$ is called the differential invariant rank and $s$ the differential invariant order of $S$. Let $\sigma: S \rightarrow \mathcal{S}^{(s+1)}$ denote the signature map from $S$ to its order $s+1$ signature, so $\sigma(z)=J^{(s+1)}(z)$ for $z \in S$ is parametrized by the restricted differential invariants of order $\leq s+1$.

Example 6.2. The simplest and most familiar example of a signature appears in the Euclidean geometry of oriented planar curves $C \subset \mathbb{R}^{2}$. The Euclidean signature curve has order $s+1=3$, and is parametrized by the two basic differential invariants - the curvature $\kappa$ and its derivative with respect to arc length, $\kappa_{s}$. The functional relationship $\kappa_{s}=H(\kappa)$ between the two uniquely determines the curve up to a Euclidean motion. This is more or less equivalent to the classical result, $[\mathbf{2 4}]$, that a Euclidean curve is uniquely characterized by curvature as a function of arc length $\kappa=\kappa(s)$. The signature curve version of this result has several advantages, of particular relevance in object recognition, [13], in that it (a) is completely local, and (b) is completely unambiguous, whereas the evaluation of the arc length $s$ requires integration along the curve, and so is not local, and depends upon the starting point, leaving a one-parameter ambiguity.

For a (generic) surface $S \subset \mathbb{R}^{3}$, the Euclidean signature has order $s+1=3$, and is parametrized by a total of six differential invariants - the Gaussian and mean curvatures along with their first order derivatives with respect to the Frenet frame, [24]. Surfaces of higher order occur when the Gaussian and mean curvatures are functionally dependent, but one of their derivatives is not.

We will call a submanifold nonsingular if it has maximal differential invariant rank $t=p$, which means that it admits $p=\operatorname{dim} S$ functionally independent restricted differential invariants. Generic submanifolds are nonsingular, and also have minimal differential invariant order, which will be either $s=n$ or $s=n+1$. The former case occurs if $\operatorname{dim} \mathrm{J}^{n}-\operatorname{dim} G \geq p$, and hence there are at least $p$ independent differential invariants of order $n$; otherwise one must go to order $s=n+1$ in order to obtain at least $p$ independent differential invariants. Higher order submanifolds are exceptional, although they are important, [19], in Ovsiannikov's method of partially invariant solutions of partial differential equations, [35]. Example 6.8 below illustrates the basic ideas.

The differential invariant rank of a submanifold is intimately related to the dimension of its symmetry group $G_{S}$. This means that the rank is an intrinsic invariant quantity, independent of the particular moving frame used to compute it.

Theorem 6.3. Let $S \subset M$ be a fully regular $p$-dimensional submanifold of differential invariant rank $t$ with respect to a moving frame $\rho^{(n)}$. Then the symmetry group $G_{S}$ is an $(r-t)$-dimensional subgroup of $G$ that acts locally freely on $S$. 
In particular, the symmetry group of a submanifold is discrete if and only if the submanifold is nonsingular. Furthermore, the number of points in the submanifold $S$ that map to a single, generic point of its signature submanifold $\mathcal{S}^{(s+1)}$ determines the number of discrete symmetries of $S$.

Definition 6.4. The index of a nonsingular submanifold $S$ of order $s$ is defined as

$$
\text { ind } S=\min \left\{\# \sigma^{-1}\{\zeta\} \mid \zeta \in \mathcal{S}^{(s+1)}\right\} \text {. }
$$

Theorem 6.5. The cardinality of the discrete symmetry group of a nonsingular submanifold equals its index: $\# G_{S}=$ ind $S$.

In other words, to count the number of symmetries of $S$, we need only determine how many points in $S$ map to one (generic) point of its signature. Incidentally, a point on the signature is non-generic if and only if it is a point of self-intersection of $\mathcal{S}^{(s+1)}$. As discussed in [13], Theorem 6.5 has important potential applications to the detection of discrete symmetries of objects in images; see $[\mathbf{9}, \mathbf{2 3}, \mathbf{4 0}, \mathbf{4 1}]$ for other methods. We will develop the symmetry-detection consequences of our results on joint signatures in a subsequent paper. See also $[\mathbf{1}, \mathbf{3 3}]$ for applications of these ideas to the classification of symmetries of polynomials in classical invariant theory.

At the other extreme, a rank 0 submanifold has all constant differential invariants, and so its signature degenerates to a single point. According to Theorem 6.3, the rank 0 submanifolds are maximally symmetric, since they admit a $p$-dimensional symmetry group. (Totally singular submanifolds may admit an even higher-dimensional symmetry group, but these are not covered by the moving frame construction.) In fact, the maximally symmetric submanifolds can be explicitly characterized as the orbits of certain subgroups of $G,[14,19,25]$.

Theorem 6.6. Let $G$ act effectively on $M$. A regular $p$-dimensional submanifold $S$ has differential invariant rank 0 if and only if it is the p-dimensional orbit, $S=H \cdot z_{0}$, of a $p$-dimensional subgroup $H=G_{S} \subset G$.

For example, in planar Euclidean geometry, the maximally symmetric curves have constant Euclidean curvature, and are the circles and straight lines. Each is the orbit of a one-parameter subgroup of $\mathrm{SE}(2)$, which also forms the symmetry group of the orbit.

The preceding symmetry results are all consequences of the fundamental equivalence theorem, which states that the signature submanifold uniquely characterizes the original submanifold up to a group transformation.

Theorem 6.7. Let $S, \bar{S} \subset M$ be regular $p$-dimensional submanifolds with respect to a moving frame map $\rho^{(n)}$. Then $S$ and $\bar{S}$ are (locally) congruent, $\bar{S}=g \cdot S$, if and only if they have the same differential invariant order $s$ and their signature manifolds of order $s+1$ are identical: $\mathcal{S}^{(s+1)}(\bar{S})=\mathcal{S}^{(s+1)}(S)$.

Example 6.8. All of the complications already occur in the simplest example equivalence of surfaces $S \subset M=\mathbb{R}^{3}$ under the translation group $G=\mathbb{R}^{3}$ acting by $z \mapsto w=z+a$. The group acts freely on $M$, and so one can construct a moving frame of 
order $n=0$ by choosing any single point $\left\{z_{0}\right\}$ as a cross-section. For simplicity, we take $z_{0}=0$, which leads to the normalization equations $w=z-a=0$, and the moving frame is $\rho(z)=a$. The group acts completely trivially on the derivatives, and so the generating differential invariants for surfaces $u=f(x, y)$ are just $u_{x}, u_{y}, u_{x x}, u_{x y}, \ldots$

A generic surface of rank $p=2$ will have functionally independent first order differential invariants $u_{x}, u_{y}$, and differential invariant order $s=n+1=1$. The signature of such a surface will be parametrized by the differential invariants of order $\leq s+1=2$, namely $u_{x}, u_{y}, u_{x x}, u_{x y}, u_{y y}$. Thus, locally, a surface $u=f(x, y)$ is uniquely determined up to translation by the functional relationships

$$
u_{x x}=H_{1}\left(u_{x}, u_{y}\right), \quad u_{x y}=H_{2}\left(u_{x}, u_{y}\right), \quad u_{y y}=H_{3}\left(u_{x}, u_{y}\right),
$$

among these five differential invariants. These dependencies will (locally) prescribe the signature surface $\mathcal{S}^{(2)}(S)$.

A surface will have rank $p=2$ and differential invariant order $s=2$ if $u_{x}$ and $u_{y}$ are functionally dependent, and so $u=f(x, y)$ is a solution to a first order partial differential equation of the form

$$
u_{y}=H\left(u_{x}\right),
$$

but at least one of the second order differential invariants $u_{x x}, u_{x y}, u_{y y}$ is not functionally dependent upon $u_{x}$. An explicit example is provided by the surface $u=\sqrt{x y}$, which has $u_{y}=1 / u_{x}$, but $u_{x x}$ is independent. Note that if we differentiate (6.3) we obtain two functional dependencies among the second order differential invariants

$$
u_{x y}=H^{\prime}\left(u_{x}\right) u_{x x}, \quad u_{y y}=H^{\prime}\left(u_{x}\right) u_{x y}=H^{\prime}\left(u_{x}\right)^{2} u_{x x} .
$$

In this case, the signature $\mathcal{S}^{(3)}(S)$ is parametrized by the order $s+1=3$ differential invariants, and, in fact, we only need to know the dependency $u_{x x x}=K\left(u_{x}, u_{x x}\right)$ since the remaining dependencies, and hence the form of $\mathcal{S}^{(3)}(S)$ are found by differentiating (6.4).

A surface will have rank 1, and hence a one-parameter group of translational symmetries, if and only if $u_{y}, u_{x x}, u_{x y}, u_{y y}$ are all functionally dependent upon $u_{x}$. The surfaces

$$
u(x, y)=h(x-a y)+b x
$$

provide (most of the) examples. All rank 1 surfaces have differential invariant order $s=1$ and hence they are characterized by the second order signature curve $\mathcal{S}^{(2)}(S)$.

Finally, a rank 0 surface has all constant differential invariants, and hence its signature degenerates to a single point. Such maximally symmetric surfaces are just the flat planes, which are the only surfaces to admit a two-dimensional translation symmetry group.

As the example indicates, in practical applications, one rarely needs all of the order $s+1$ differential invariants $I^{(s+1)}$ that parametrize the signature $\mathcal{S}^{(s+1)}$ in order to uniquely characterize $S$ up to congruence. Recurrence relations and syzygies among the differentiated invariants can often be used to eliminate many redundant invariants. In general, there are two types of syzygies. First are the universal syzygies, which hold directly among the differential invariants, and so do not depend on the particularities of the submanifold $S$. Actually, if we employ the fundamental normalized differential invariants $I^{(s+1)}$, then the 
only universal syzygies are those given by the phantom invariants being constant. However, there may be good geometrical reasons for considering alternative sets of differential invariants derived from the fundamental ones, which do admit some universal syzygies see the discussion at the end of Example 8.2. As far as the equivalence properties of $S$ go, then, we can ignore all differential invariants arising from such universal syzygies. Second are the particular syzygies that arise because we have restricted the differential invariants to the submanifold $S$. Indeed, since there are at most $p$ functionally independent functions on a $p$-dimensional submanifold, most of the restricted differential invariants will be related via (local) syzygies. The particular syzygies will serve to characterize the signature, and thereby uniquely characterize $S$ up to equivalence.

Many of the syzygies can be deduced as a direct consequence of others, and so one only requires a few fundamental syzygies in order to completely characterize the signature. Consider a syzygy

$$
\Phi\left(J_{1}, \ldots, J_{p}\right)=0
$$

among the restricted differential invariants — which may be either universal or particular to the submanifold $S$. If we apply the invariant differential operator $\mathcal{D}_{i}$ to $(6.5)$, we deduce a new syzygy

$$
\sum_{k=1}^{p} \mathcal{D}_{i} J_{k} \cdot \frac{\partial \Phi}{\partial J_{k}}\left(J_{1}, \ldots, J_{p}\right)=0
$$

among the differentiated invariants. The derived syzygy (6.6) is completely determined by the original syzygy (6.5), and hence is irrelevant for the unique specification of the signature of $S$. (An example of this computation appears in (6.3), (6.4) in the example.) A reduced signature of $S$ is thereby constructed by discarding all the differential invariants whose values are determined by the universal syzygies and all such derived syzygies. All the syzygies of the resulting minimal system of differential invariants are now particular to $S$, and serve to uniquely characterize it up to equivalence: any congruent submanifold $\bar{S}=g \cdot S$ must have the the same reduced signature, meaning the same minimal system of restricted differential invariants and the same particular syzygies. Practical illustrations of this reduction technique will appear in our examples.

\section{Joint Differential Invariants.}

Let us now adapt our general moving frame constructions to the case of primary concern in this paper - Cartesian product actions of groups and their prolongations. Let $G$ act on $M$, and consider the induced action (3.1) on the Cartesian product space $M^{\times n}$ for some $n>1$. If $S \subset M$ is an ordinary $p$-dimensional submanifold, its $n$-fold Cartesian product is the $(n p)$-dimensional submanifold

$$
S^{\times n}=S \times \cdots \times S \subset M^{\times n}
$$

Two submanifolds $S, \bar{S} \subset M$ are congruent, so $\bar{S}=g \cdot S$ for some $g \in G$, if and only if their Cartesian products are: $\bar{S}^{\times n}=g \cdot S^{\times n}$. The symmetry group of the product $S^{\times n}$ 
is the same as the symmetry group $G_{S} \subset G$ of $S$. If $S$ is not totally singular, and so $G_{S}$ acts locally freely on $S$, then

$$
\operatorname{dim} G_{S}=k \leq p<n p=\operatorname{dim} S^{\times n} .
$$

More generally, two ordered $n$-tuples of $p$-dimensional submanifolds $\left(S^{1}, \ldots, S^{n}\right)$ and $\left(\bar{S}^{1}, \ldots, \bar{S}^{n}\right)$ are simultaneously congruent, so $\bar{S}^{k}=g \cdot S^{k}, k=1, \ldots, n$, under the same group element $g \in G$ if and only if their Cartesian products $\mathbf{S}=S^{1} \times \cdots \times S^{n}$ and $\overline{\mathbf{S}}=\bar{S}^{1} \times \cdots \times \bar{S}^{n}$ are congruent submanifolds of $M^{\times n}$, meaning that $\overline{\mathbf{S}}=g \cdot \mathbf{S}$.

Proposition 7.1. A Cartesian product $\mathbf{S}=S^{1} \times \cdots \times S^{n} \subset M^{\times n}$ of $p$-dimensional submanifolds $S^{1}, \ldots, S^{n} \subset M$ has symmetry group $G_{\mathbf{S}}=G_{S^{1}} \cap \cdots \cap G_{S^{n}}$ If at least one of the $S^{\nu}$ is regular, then $\operatorname{dim} G_{\mathbf{S}} \leq p$. In particular, $\mathbf{S}$ has a $p$-dimensional symmetry group if and only if each $S^{\nu}=H \cdot z^{\nu}, \nu=1, \ldots, n$, is a regular orbit of the same $p$-dimensional subgroup $H=G_{\mathbf{S}} \subset G$.

Remark: Proposition 7.1 implies that product submanifolds can never be maximally symmetric in $M^{\times n}$ since their symmetry groups have dimension at most $p$ which is strictly less than their own dimension. Consequently, the joint invariants (and, a fortiori the joint differential invariants) of a product submanifold of $M^{\times n}$ cannot all be constant!

Not every submanifold of $M^{\times n}$ is a Cartesian product. Indeed, the "mixed jet derivatives" - meaning derivatives with respect to the coordinates on different copies of $M$ must all vanish on product submanifolds. Moreover, these conditions serve to uniquely prescribe the product jet subbundle. More formally, the $k^{\text {th }}$ order jets of product submanifolds $\mathbf{S}$ form submanifolds of the $k^{\text {th }}$ order jet bundle $\mathrm{J}^{k}\left(M^{\times n}, n p\right)$. On the other hand, we can identify

$$
\mathrm{j}_{k} \mathbf{S}=\mathrm{j}_{k} S^{1} \times \cdots \times \mathrm{j}_{k} S^{n} \subset \mathrm{J}^{k}(M, p) \times \cdots \times \mathrm{J}^{k}(M, p)=\mathrm{J}^{k}(M, p)^{\times n}=\left(\mathrm{J}^{k}\right)^{\times n}
$$

as a submanifold of the Cartesian product jet bundle. The latter forms the subbundle

$$
\mathrm{J}^{k}(M, p)^{\times n} \subset \mathrm{J}^{k}\left(M^{\times n}, n \cdot p\right)
$$

which is traced out by the $k$-jets of $n$-fold product submanifolds $\mathbf{S}=S^{1} \times \cdots \times S^{n} \subset M^{\times n}$.

Remark: One may also consider more general Cartesian products $\mathrm{J}^{k_{1}} \times \cdots \times \mathrm{J}^{k_{n}}$ of different order jet bundles, named "multi-space" in [16]. However, any such product can be obtained by projection from a common jet space $\left(\mathrm{J}^{k}\right)^{\times n}$, where $k \geq \max k_{\nu}$, and so we do not lose any generality by only considering Cartesian products of identical jet bundles.

The product action of the Lie group $G$ on $M^{\times n}$ induces a prolonged action on $\mathrm{J}^{k}\left(M^{\times n}, n p\right)$ which preserves the product subbundle $\left(\mathrm{J}^{k}\right)^{\times n}$, and coincides with the $n$ fold Cartesian product of the action on $\mathrm{J}^{k}(M, p)$. The invariants of the induced action of $G$ on $\left(\mathrm{J}^{k}\right)^{\times n}$ are known as joint differential invariants, [19], or, in the computer vision literature, semi-differential invariants, $[\mathbf{4}, \mathbf{3 8}, \mathbf{3 9}]$, or multi-local invariants, $[\mathbf{1 6}]$. Combining the prolongation and product constructions of moving frames leads immediately to a general moving frame algorithm for completely classifying joint differential invariants. 
If $G$ acts freely on an open subset of $\left(\mathrm{J}^{k}\right)^{\times n}$, then, according to Theorem 5.1 , there exists a complete system of fundamental joint differential invariants of order $\leq k+1$ such that every other joint differential invariant can be obtained by invariant differentiation. Note that, for $p$-dimensional submanifolds of $M$, there are $n \cdot p$ distinct invariant differentiations $\mathcal{D}_{i}^{\nu}$, where $\nu=1, \ldots, n$ and $i=1, \ldots, p$, where $\mathcal{D}_{1}^{\nu}, \ldots, \mathcal{D}_{p}^{\nu}$ only involve differentiation with respect to the coordinates belonging to the $\nu^{\text {th }}$ copy of $M$ in $M^{\times n}$.

Remark: Mixed invariant derivatives of joint invariants do not necessarily vanish, even when restricted to product submanifolds.

The fundamental equivalence and symmetry Theorems 6.7 and 6.3 apply as stated to product submanifolds. The joint differential invariant rank $t$ of a submanifold $S \subset M$ (or, more generally, an ordered $n$-tuple of submanifolds $S^{1}, \ldots, S^{n} \subset M$ ) is the number of functionally independent joint differential invariants on the product. Since the symmetry group of the product submanifold $S^{\times n}$ is the same as the symmetry group of $S$, Theorem 6.3 implies that the $n$-point joint differential invariant rank is related to the usual differential invariant rank of $S$ according to the equation

$$
p-\operatorname{rank} S=\operatorname{dim} G_{S}=\operatorname{dim} G_{S^{\times n}}=n p-\operatorname{rank} S^{\times n} .
$$

This relation predicts the number of functionally independent joint differential invariants on $S$.

The joint differential invariant order $s$ of a product submanifold $\mathbf{S}=S^{1} \times \cdots \times S^{n}$ is the minimal order required to produce a generating system of functionally independent joint differential invariants. Typically, the more points used, the lower the joint differential invariant order. In particular, if $G$ acts freely on $M^{\times n}$, then the joint differential invariant order of a generic product submanifold $\mathbf{S}$ is $s=0$, meaning that we can find a complete system of $t=\operatorname{rank} \mathbf{S}$ functionally independent joint invariants on $\mathbf{S}$. In the generic case, when $\mathbf{S}$ is nonsingular and only has discrete symmetries, $\operatorname{rank} \mathbf{S}=n p$. If $\mathbf{S} \subset M^{\times n}$ has joint differential invariant order 0 , then Theorems 6.3, 6.5, and 6.7 imply that the equivalence and symmetry properties of $\mathbf{S}$ are completely determined by the first order joint signature submanifold $\mathcal{S}^{(1)}(\mathbf{S})$, which is parametrized by the joint invariants and the first order joint differential invariants.

In all the cases considered here, provided we use enough points on the submanifold, all the joint differential invariants can be expressed as invariant derivatives of the joint invariants. In many, but not all cases, it suffices to have one nontrivial joint invariant in order to generate the joint differential invariants. Moreover, all the signature syzygies turn out to be differential consequences, as in (6.6), of the syzygies among the joint invariants. Both of these facts are consequences of the particular recurrence formulae for the group. So far we have not determined how general this result might be.

If $\operatorname{dim} G=r$ and $\operatorname{dim} M=m$, then we find that, in all our examples, $G$ acts freely on an open subset of $M^{\times n}$ provided $n m \geq r$, and so there are $n m-r$ functionally independent joint invariants. Moreover, if $n m-r>n p$, then there will be at least one zero ${ }^{\text {th }}$ order syzygy among the restricted joint invariants. Consequently, a necessary condition for the restricted signature of a generic $n$-point submanifold be defined solely by joint invariants 
is

$$
n \geq \frac{r}{m-p}+1
$$

It should be emphasized that this count applies to generic submanifolds, and exceptional submanifolds of higher order - which require additional points in order to construct a proper signature - as well as exceptional group actions can occur.

\section{Joint Signatures for Curves.}

We now illustrate the general theory with a number of particular examples, chosen for their relevance to basic issues in geometry of curves and surfaces as well as potential applications in computer vision. Needless to say, this is only a very small fraction of the cases amenable to these techniques, and we will report on other, more complicated, situations in later publications. See also $[\mathbf{1}, \mathbf{2 7}]$ for other non-traditional applications of the moving frame method.

Let us look more closely at the joint signature submanifolds in the case of planar curves. Consider an $r$-dimensional Lie group $G$ acting effectively on an open subset of $M=\mathbb{R}^{2}$. If, as happens in all our examples, $G$ acts freely on $M^{\times n}$ for $2 n \geq r$, then there exist $2 n-r$ functionally independent $n$-point joint invariants. Moreover, there are $n$ additional joint differential invariants at each order $k \geq 1$. In the planar cases considered here, as long as $2 n>r$, the joint differential invariants are all obtained from the $2 n-r$ joint invariants by invariant differentiation. Therefore, the $k^{\text {th }}$ order signature submanifold is parametrized by $(k+2) n-r$ independent joint differential invariants. The same is true even when the joint action on $M^{\times n}$ is not free, as long as $k \geq \frac{r}{n}-2$ is sufficiently large to ensure that $G$ acts locally freely on $\left(\mathrm{J}^{k}\right)^{\times n}$.

On the other hand, an $n$-point curve defines a product submanifold $C^{\times n} \subset M^{\times n}$ and so, generically, one requires at least $n+1$ invariants in order that at least one nontrivial syzygy exist. Therefore, a zero ${ }^{\text {th }}$ order joint signature submanifold is possible only when $2 n-r \geq n+1$, i.e., when $n \geq r+1$, in accordance with the general inequality (7.2).

Proposition 8.1. Let $G$ be an $r$-dimensional group acting on planar curves. Then the order $k$ of the $n$-point joint signature submanifold of a curve will bounded from below by the inequality $k \geq \frac{r+1}{n}-1$.

Let us now consider some particular geometric examples.

Example 8.2. Euclidean joint differential invariants. Consider the proper Euclidean group $\mathrm{SE}(2)$ acting on oriented curves in the plane $M=\mathbb{R}^{2}$, as in Example 4.1. (The resolution of the sign ambiguities in the cases of non-oriented curves and/or the full Euclidean group $\mathrm{E}(2)$ are left to the reader.) We begin with the case of two-point invariants, corresponding to the Cartesian product action on $M^{\times 2} \simeq \mathbb{R}^{4}$. Taking the simplest cross-section ${ }^{\dagger}$

$\dagger$ We use a slightly different cross-section — better suited to the required computations than was used in Example 3.6. 


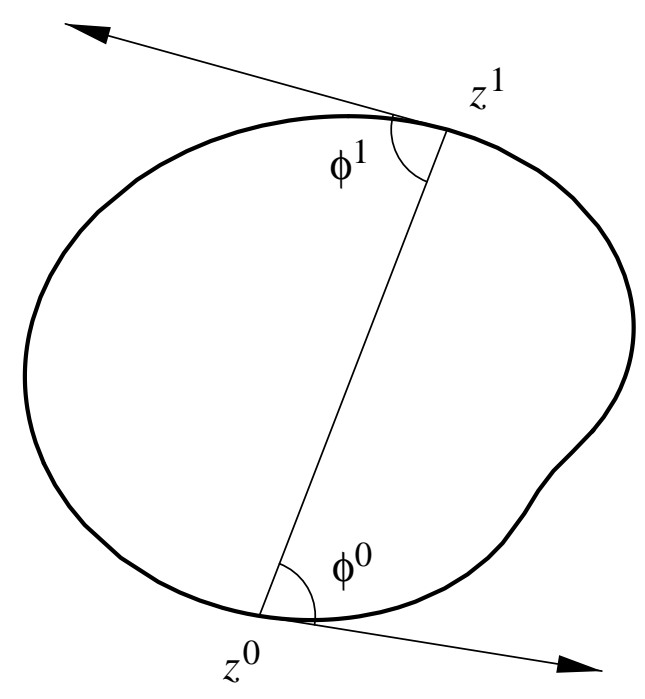

Figure 6. Joint Euclidean Angles.

$x^{0}=u^{0}=x^{1}=0, u^{1}>0$ leads to the normalization equations

$$
y^{0}=0, \quad v^{0}=0, \quad y^{1}=0 .
$$

Solving, we obtain a left moving frame

$$
\widetilde{\theta}=-\cot ^{-1}\left(\frac{u^{1}-u^{0}}{x^{1}-x^{0}}\right), \quad \widetilde{a}=x^{0}, \quad \widetilde{b}=u^{0},
$$

along with the fundamental interpoint distance invariant

$$
v^{1} \longmapsto I=\left\|z^{1}-z^{0}\right\|,
$$

reproducing the results of Example 3.6 in the planar case. Substituting (8.2) into the prolongation formulae (4.11) leads to the explicit expressions

$$
v_{y}^{k} \longmapsto J_{k}=-\frac{\left(z^{1}-z^{0}\right) \cdot z_{t}^{k}}{\left(z^{1}-z^{0}\right) \wedge z_{t}^{k}}, \quad v_{y y}^{k} \longmapsto K_{k}=-\frac{\left\|z^{1}-z^{0}\right\|^{3}\left(z_{t}^{k} \wedge z_{t t}^{k}\right)}{\left[\left(z^{1}-z^{0}\right) \wedge z_{t}^{0}\right]^{3}},
$$

for the normalized first and second order joint differential invariants. We require that the chord $z^{1}-z^{0}$ connecting the points not be tangent to the curve at either endpoint. The first order two-point joint differential invariants are therefore

$$
J_{0}=-\cot \phi^{0}, \quad J_{1}=+\cot \phi^{1},
$$

where $\phi^{k}=\Varangle\left(z^{1}-z^{0}, z_{t}^{k}\right)$ denotes the angle from the chord to the tangent vector at $z^{k}$, as indicated in Figure 6 . The modified second order joint differential invariant

$$
\widehat{K}_{0}=-\left\|z^{1}-z^{0}\right\|^{-3} K_{0}=\frac{z_{t}^{0} \wedge z_{t t}^{0}}{\left[\left(z^{1}-z^{0}\right) \wedge z_{t}^{0}\right]^{3}}
$$




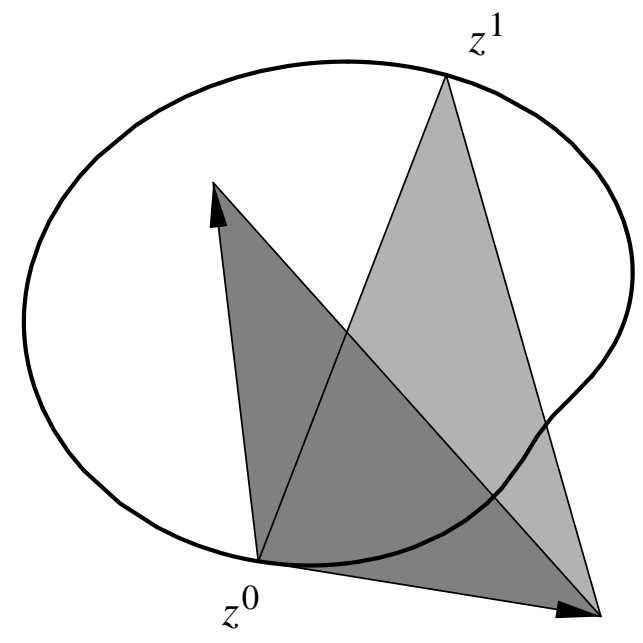

Figure 7. Second Order Joint Differential Invariant.

equals the ratio of the area of triangle whose sides are the first and second derivative vectors $z_{t}^{0}, z_{t t}^{0}$ at the point $z^{0}$ over the cube of the area of triangle whose sides are the chord from $z^{0}$ to $z^{1}$ and the tangent vector at $z^{0}$; see Figure 7 . This joint differential invariant is, interestingly, invariant under the equi-affine group; see the following example.

On the other hand, we can construct the joint differential invariants by invariant differentiation of the basic distance invariant $I$. The normalized invariant one-forms, that assume the role of "joint arc length forms", are

$$
d y^{k}=\left(x_{t}^{k} \cos \theta-u_{t}^{k} \sin \theta\right) d t^{k} \quad \longmapsto \quad \omega^{k}=-\frac{\left(z^{1}-z^{0}\right) \wedge z_{t}^{k}}{\left\|z^{1}-z^{0}\right\|} d t^{k},
$$

with corresponding invariant differential operators

$$
D_{y^{k}} \quad \longmapsto \quad \mathcal{D}_{k}=-\frac{\left\|z^{1}-z^{0}\right\|}{\left(z^{1}-z^{0}\right) \wedge z_{t}^{k}} D_{t^{k}} .
$$

It is not difficult to directly establish the recurrence formulae expressing the differentiated invariants in terms of the fundamental normalized joint differential invariants. However, the general infinitesimal algorithm presented in Section 5 is also available, and, in more complicated examples, almost essential for obtaining the syzygies without undue computational effort. To prepare for such computations (which, for brevity, we shall subsequently suppress), we present the full details in this relatively simple example.

In general, to construct the recurrence formulae for the $n$-point joint differential invariants of order $k$, one introduces the $n$-fold joint Lie matrix $\mathbf{L}_{k}^{\times n}$ obtained by juxtaposing $n$ copies of the ordinary order $k$ Lie matrix (5.6), each copy being indexed by the corresponding point on the curve (or submanifold). In the case of two-point joint Euclidean 
invariants, one starts with the second order (for simplicity - higher order recurrences are readily found by the same algorithm) joint Lie matrix

$$
\mathbf{L}_{2}^{\times 2}=\left(\begin{array}{cccccccc}
1 & 0 & 0 & 0 & 1 & 0 & 0 & 0 \\
0 & 1 & 0 & 0 & 0 & 1 & 0 & 0 \\
-u^{0} & x^{0} & 1+\left(u_{x}^{0}\right)^{2} & 3 u_{x}^{0} u_{x x}^{0} & -u^{1} & x^{1} & 1+\left(u_{x}^{1}\right)^{2} & 3 u_{x}^{1} u_{x x}^{1}
\end{array}\right),
$$

based on (5.10). For the normalization (8.2), (8.3), the invariantized joint Lie matrix $\mathbf{I}_{2}^{\times 2}=\iota\left(\mathbf{L}_{2}^{\times 2}\right)$ is

$$
\mathbf{I}_{2}^{\times 2}=\left(\begin{array}{cccccccc}
1 & 0 & 0 & 0 & 1 & 0 & 0 & 0 \\
0 & 1 & 0 & 0 & 0 & 1 & 0 & 0 \\
0 & 0 & 1+J_{0}^{2} & 3 J_{0} K_{0} & -I & 0 & 1+J_{1}^{2} & 3 J_{1} K_{1}
\end{array}\right) .
$$

Since in (8.1) we are normalizing the variables $x^{0}, u^{0}, x^{1}$ that correspond to the first, second and fifth columns of (8.9), the algorithm requires us to perform a Gauss-Jordan row reduction that converts this particular $3 \times 3$ minor of $\mathbf{I}_{2}^{\times 2}$ into the identity matrix; the net result is

$$
\mathbf{K}_{2}^{\times 2}=\left(\begin{array}{cccccccc}
1 & 0 & \left(1+J_{0}^{2}\right) / I & 3 J_{0} K_{0} / I & 0 & 0 & \left(1+J_{1}^{2}\right) / I & 3 J_{1} K_{1} / I \\
0 & 1 & 0 & 0 & 0 & 1 & 0 & 0 \\
0 & 0 & -\left(1+J_{0}^{2}\right) / I & -3 J_{0} K_{0} / I & 1 & 0 & -\left(1+J_{1}^{2}\right) / I & -3 J_{1} K_{1} / I
\end{array}\right) .
$$

On the other hand, the total derivative matrix corresponding to our normalization variables $x^{0}, u^{0}, x^{1}$ and its invariantized counterpart are

$$
\mathbf{Z}^{\times 2}=\left(\begin{array}{ccc}
1 & u_{x}^{0} & 0 \\
0 & 0 & 1
\end{array}\right), \quad \mathbf{W}^{\times 2}=\iota\left(\mathbf{Z}^{\times 2}\right)=\left(\begin{array}{ccc}
1 & J_{0} & 0 \\
0 & 0 & 1
\end{array}\right) .
$$

Note that we are explicitly restricting our attention to product curves $C_{0} \times C_{1} \subset M^{\times 2}$, for which all the mixed derivatives, including all mixed-derivative normalized invariants, will automatically vanish. The second order correction matrix is given by the product $\mathbf{M}_{2}^{\times 2}=\mathbf{W}^{\times 2} \cdot \mathbf{K}_{2}^{\times 2}$, and equals

$$
\mathbf{M}_{2}^{\times 2}=\left(\begin{array}{cccccccc}
1 & J_{0} & \left(1+J_{0}^{2}\right) / I & 3 J_{0} K_{0} / I & 0 & J_{0} & \left(1+J_{1}^{2}\right) / I & 3 J_{1} K_{1} / I \\
0 & 0 & -\left(1+J_{0}^{2}\right) / I & -3 J_{0} K_{0} / I & 1 & 0 & -\left(1+J_{1}^{2}\right) / I & -3 J_{1} K_{1} / I
\end{array}\right) .
$$

The entries of $\mathbf{M}_{2}^{\times 2}$ are the correction terms in the basic recurrence formulae:

$$
\begin{array}{rlrl}
\mathcal{D}_{0} I & =-J_{0}, & \mathcal{D}_{1} I=J_{1}, \\
\mathcal{D}_{0} J_{0} & =K_{0}-\frac{1+J_{0}^{2}}{I}, & & \mathcal{D}_{1} J_{0}=\frac{1+J_{0}^{2}}{I}, \\
\mathcal{D}_{0} J_{1} & =-\frac{1+J_{1}^{2}}{I}, & & \mathcal{D}_{1} J_{1}=K_{1}+\frac{1+J_{1}^{2}}{I}, \\
\mathcal{D}_{0} K_{0} & =L_{0}-\frac{3 J_{0} K_{0}}{I}, & & \mathcal{D}_{1} K_{0}=\frac{3 J_{0} K_{0}}{I}, \\
\mathcal{D}_{0} K_{1} & =-\frac{3 J_{1} K_{1}}{I}, & \mathcal{D}_{1} K_{1}=L_{1}+\frac{3 J_{1} K_{1}}{I} .
\end{array}
$$


Here $v_{y y y}^{k} \longmapsto L_{k}$ are the normalized joint differential invariants coming from the third order derivatives. These formulae imply that all of the joint differential invariants can be obtained from the basic distance invariant by invariant differentiation. Therefore, we have proved the following result, cf. [19].

Proposition 8.3. Every two-point Euclidean joint differential invariant is a function of the interpoint distance $I=\left\|z^{1}-z^{0}\right\|$ and its invariant derivatives with respect to (8.8).

For example, consider the Euclidean curvature

$$
\kappa^{0}=\frac{z_{t}^{0} \wedge z_{t t}^{0}}{\left\|z_{t}^{0}\right\|^{3}}=\frac{u_{x x}^{0}}{\left(1+\left(u_{x}^{0}\right)^{2}\right)^{3 / 2}}
$$

at the point $z^{0}$. To determine how to write it in terms of the normalized and differentiated invariants, we can apply our invariantization procedure and (8.12):

$$
\kappa^{0}=\iota\left(\kappa^{0}\right)=\frac{K_{0}}{\left(1+J_{0}^{2}\right)^{3 / 2}}=-\frac{\mathcal{D}_{0}^{2} I}{\left(1+\left(\mathcal{D}_{0} I\right)^{2}\right)^{3 / 2}}+\frac{1}{I \sqrt{1+\left(\mathcal{D}_{0} I\right)^{2}}} .
$$

A generic product curve $\mathbf{C}=C^{0} \times C^{1} \subset M^{\times 2}$ has joint differential invariant rank $2=\operatorname{dim} \mathbf{C}$. Since there is only one joint invariant, the minimal joint differential invariant order of $\mathbf{C}$ is $s=1$, and the required signature $\mathcal{S}^{(2)}(\mathbf{C})$ will be parametrized by the joint differential invariants $I, J_{0}, J_{1}, K_{0}, K_{1}$ of order $\leq 2$. Since $\mathcal{S}^{(2)}(\mathbf{C})$ is, by assumption, a two-dimensional submanifold, there must exist a particular (local) syzygy

$$
\Phi\left(I, J_{0}, J_{1}\right)=0
$$

among the three first order joint differential invariants. Differentiating (8.13) and using the recurrence formulae (8.12), we find

$$
\begin{array}{r}
-J_{0} \frac{\partial \Phi}{\partial I}+\left(K_{0}-\frac{1+J_{0}^{2}}{I}\right) \frac{\partial \Phi}{\partial J_{0}}-\left(\frac{1+J_{1}^{2}}{I}\right) \frac{\partial \Phi}{\partial J_{1}}=0 \\
J_{1} \frac{\partial \Phi}{\partial I}+\left(\frac{1+J_{0}^{2}}{I}\right) \frac{\partial \Phi}{\partial J_{0}}+\left(K_{1}+\frac{1+J_{1}^{2}}{I}\right) \frac{\partial \Phi}{\partial J_{1}}=0 .
\end{array}
$$

Thus, the syzygies for second order joint differential invariant $K_{0}, K_{1}$ are uniquely determined, provided

$$
\frac{\partial \Phi}{\partial J_{0}} \neq 0 \quad \text { and } \quad \frac{\partial \Phi}{\partial J_{1}} \neq 0 .
$$

Therefore, the surface parametrized by the first order joint differential invariants $I, J_{0}, J_{1}$ can be used as the reduced signature set to characterize such (generic) product curves. Failure of (8.14) at isolated points is not a problem (at least in the analytic category) since one can use analytic continuation to characterize the product curve. In general, rank 2 product curves admit only a discrete symmetry group, whose cardinality equals the signature index, meaning the number of pairs of points $\left(z^{0}, z^{1}\right) \in C^{0} \times C^{1}$ at which all three invariants have the same (generic) value. 
Suppose the first order signature degenerates to a one-dimensional curve, locally given by

$$
J_{0}=-\cot \phi^{0}=\Phi_{0}(I), \quad J_{1}=\cot \phi^{1}=\Phi_{1}(I) .
$$

Differentiating these two syzygies and using (8.12) leads to the four derived syzygies

$$
\begin{array}{ll}
K_{0}=-J_{0} \Phi_{0}^{\prime}(I)+\frac{1+J_{0}^{2}}{I}, & \frac{1+J_{0}^{2}}{I}=J_{1} \Phi_{0}^{\prime}(I), \\
K_{1}=J_{1} \Phi_{1}^{\prime}(I)-\frac{1+J_{1}^{2}}{I}, & -\frac{1+J_{1}^{2}}{I}=-J_{0} \Phi_{1}^{\prime}(I) .
\end{array}
$$

The first of each pair prove that the second order joint differential invariants $K_{0}, K_{1}$ are also functionally dependent upon $I$, and are automatically determined by the syzygies (8.15). Therefore, the second order signature is also a one-dimensional curve. This proves that there cannot be any product curves of joint differential invariant order $s=2$.

According to Theorem 6.3, the product curve admits a one-parameter symmetry group if and only if its signature is a one-dimensional curve. Proposition 7.1 implies that this occurs if and only if the two curves are orbits of the same one-parameter subgroup. Therefore, the two curves are either two parallel straight lines or two concentric circles. The signature functions $\Phi_{0}, \Phi_{1}$ in (8.15) are not arbitrary: there is a two-parameter family of such product curves, governed by the two radii of the concentric circles. According to the second and fourth derived syzygies in (8.16), the parametrization (8.15) of the signature curve must solve the coupled pair of nonlinear first order ordinary differential equations

$$
\frac{d J_{0}}{d I}=\frac{1+J_{0}^{2}}{I J_{1}}, \quad \frac{d J_{1}}{d I}=\frac{1+J_{1}^{2}}{I J_{0}} .
$$

In particular, if the circles are identical, $C_{1}=C_{2}$, then $J_{0}=-J_{1}$, and the general solution to $(8.17)$ is

$$
1+J_{0}^{2}=\frac{4 r^{2}}{I^{2}}
$$

where $r$ is the radius of the circle. This relation can be verified directly using the Law of Cosines, as illustrated in Figure 8. The corresponding figure for concentric, non-coincident circles will produce the general solution to the nonlinear system of ordinary differential equations (8.17).

Theorem 8.4. A curve $C$ or, more generally, a pair of curves $C_{0}, C_{1} \subset \mathbb{R}^{2}$, is uniquely determined up to a Euclidean transformation by its reduced signature, which is parametrized by the first order joint differential invariants $I, J_{0}, J_{1}$. The curve(s) have a one-dimensional symmetry group if and only if their signature is a one-dimensional curve; otherwise the signature is a two-dimensional surface, and the curve(s) have only discrete symmetries.

Remark: As remarked above, a regular product curve cannot have a two-dimensional symmetry group, or, equivalently, a zero-dimensional signature. Indeed, the only twodimensional subgroup of $\mathrm{SE}(2)$ is the translations $\mathbb{R}^{2}$, and so by Cartan's Theorem 6.6, the only surfaces with all constant differential invariants are the orbits of $\mathbb{R}^{2}$, which are the affine planes $\left\{z^{0}-z^{1}=a\right\} \subset M^{\times 2}$. But these are not product curves, and so are not relevant to our discussion. 


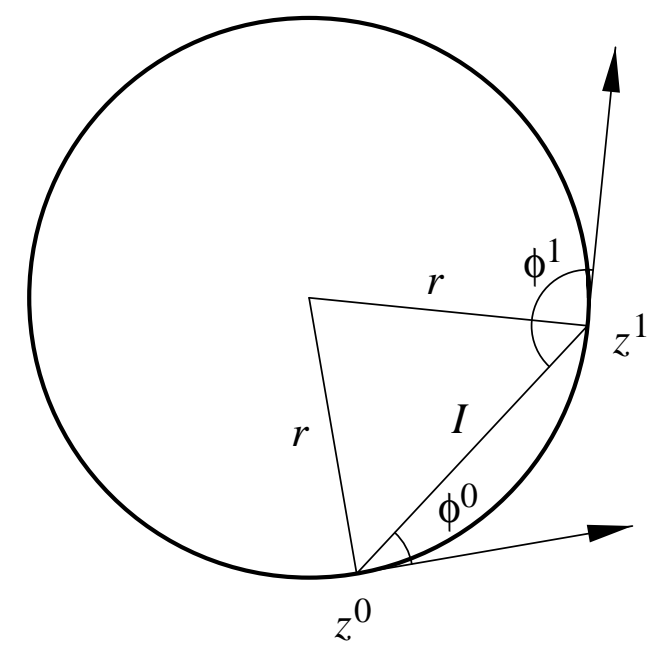

Figure 8. Triangle in Circle.

Next, consider an $n$-point Euclidean curve for $n>2$. Using the two-point moving frame (8.2), the additional joint invariants are

$$
y^{k} \longmapsto H_{k}=\left\|z^{k}-z^{0}\right\| \cos \psi^{k}, \quad v^{k} \longmapsto I_{k}=\left\|z^{k}-z^{0}\right\| \sin \psi^{k},
$$

where $\psi^{k}=\Varangle\left(z^{k}-z^{0}, z^{1}-z^{0}\right)$. Therefore, a complete system of joint invariants for $\operatorname{SE}(2)$ consists of the angles $\psi^{k}, k \geq 2$, and distances $\left\|z^{k}-z^{0}\right\|, k \geq 1$. The other distances $\left\|z^{i}-z^{j}\right\|$ and angles $\Varangle\left(z^{i}-z^{j}, z^{k}-z^{l}\right)$ can all be re-expressed as functions of these particular ones.

Conversely, one can recover the absolute values of the angles $\left|\psi^{k}\right|$ solely from knowledge of the distances $\left\|z^{i}-z^{j}\right\|$, which are full Euclidean invariants. The sign of an individual angle $\psi^{k}$ is only a proper Euclidean invariant since it can be changed by a reflection. However, the reflection will simultaneously reverse all of the signs of the angles, and so the product of any two signs is a full Euclidean invariant. It is not hard to see that knowledge of all the distances $\left\|z^{i}-z^{j}\right\|$ uniquely specifies all these joint angle sign invariants; see [33; Chapter 8]. Therefore, the distances provide a complete system of joint invariants of $\mathrm{E}(2)$, while in the orientation-preserving case of $\mathrm{SE}(2)$, one requires one signed angle, or, equivalently, one signed triangular area to build a complete list.

The first order differential invariants are the $J_{k}$ defined in (8.4). Application of our infinitesimal algorithm produces the recurrence formulae

$$
\begin{array}{lll}
\mathcal{D}_{0} I=-J_{0}, & \mathcal{D}_{1} I=J_{1}, & \\
\mathcal{D}_{0} I_{k}=-J_{0}-\frac{H_{k}}{I}, & \mathcal{D}_{1} I_{k}=\frac{H_{k}}{I}, & \mathcal{D}_{k}\left(I_{k}\right)=J_{k}, \quad k \geq 2, \\
\mathcal{D}_{0} H_{k}=\frac{I_{k}}{I}-1, & \mathcal{D}_{1} H_{k}=-\frac{I_{k}}{I}, & \mathcal{D}_{k}\left(H_{k}\right)=1 .
\end{array}
$$


All other derivatives, i.e., $\mathcal{D}_{l}\left(I_{k}\right)$ and $\mathcal{D}_{l}\left(H_{k}\right)$ for $l \neq 0,1, k$, are zero. Note that the joint differential invariants can all be found by invariant differentiation of the joint invariants $H_{k}, I_{k}$. In fact, one only needs the joint invariants $I, I_{2}, I_{3}, \ldots$, to generate the rest, since derivatives of the $I_{k}$ yield the joint invariants $H_{k}$. Therefore, we have generalized Proposition 8.3 and established a "First Main Theorem" for joint Euclidean differential invariants.

Theorem 8.5. If $n \geq 2$, then every $n$-point joint Euclidean differential invariant is a function of the interpoint distances $\left\|z^{i}-z^{j}\right\|$ and, in the case of $\mathrm{SE}(2)$, one signed triangular area $V(0,1,2)$, along with their derivatives with respect to the invariant differential operators $\mathcal{D}_{k}$ given in (8.8).

In the particular case of three-point curves, the product $C^{\times 3} \subset M^{\times 3} \simeq \mathbb{R}^{6}$ is threedimensional. There are three independent joint invariants, namely the interpoint distances. Let

$$
T^{3}=\{(a, b, c) \mid a \geq 0, b \geq 0, c \geq 0, a+b \geq c, a+c \geq b, b+c \geq a\} \subset \mathbb{R}_{+}^{3}
$$

denote the space of planar isometric triangles. For a generic curve, the three joint invariants will cover a subdomain $\mathcal{D}_{C} \subset T^{3}$, namely the set of triangles which can be inscribed in the curve. (If the curve is closed, $\mathcal{D}_{C}$ is bounded.) One therefore cannot recover the local geometry of the curve from $\mathcal{D}_{C}$ - although an interesting question is to what extent its boundary $\partial \mathcal{D}_{C}$ determines $C$. This means that generic three-pointed Euclidean curves still require a first order signature.

To create a Euclidean signature set based entirely on zero ${ }^{\text {th }}$ order joint invariants, we must use at least four points on our curve. Theorem 3.7 implies that the six interpoint distances

$$
\begin{array}{lll}
a=\left\|z^{1}-z^{0}\right\|, & b=\left\|z^{2}-z^{0}\right\|, & c=\left\|z^{3}-z^{0}\right\|, \\
d=\left\|z^{2}-z^{1}\right\|, & e=\left\|z^{3}-z^{1}\right\|, & f=\left\|z^{3}-z^{2}\right\| .
\end{array}
$$

are the fundamental joint invariants, as illustrated in Figure 1. As remarked in the introduction, these distances satisfy the universal Cayley-Menger syzygy (1.3), which can be solved for one of the distances, say $f$, in terms of the other five. There are two distinct solutions, corresponding to the two possible four-point configurations shown in Figure 9. Note that the triangle with vertices $z^{0}, z^{1}, z^{3}$ has been reflected through the line connecting $z^{0}$ and $z^{1}$, which implies that the first five distances $a, b, c, d, e$ are the same in both configurations. Therefore, adopting the more natural distance invariants in place of the moving frame angular invariants requires us to compute the "extended signature" $\widehat{\mathcal{S}} \subset \mathbb{R}^{6}$ parametrized by all six distances (8.19). There are, in fact, good practical reasons for taking this approach. The extended signature clearly exposes certain discrete symmetry properties of the signature of a multi-pointed curve. Namely, the symmetric group $\mathbb{S}^{4}$ acts on $C^{\times 4}$ by permuting the points. Since this does not affect the interpoint distances, the extended signature $\widehat{\mathcal{S}}$ is invariant under the induced action of $\mathbb{S}^{4}$ on $\mathbb{R}^{6}$. For example, interchanging $z^{0} \leftrightarrow z^{1}$ induces the distance permutation $b \leftrightarrow d, c \leftrightarrow e$. (This corresponds to the natural embedding $\mathbb{S}^{4} \hookrightarrow \mathbb{S}^{6}$ induced by the action of $\mathbb{S}^{4}$ on the six different two-element subsets of a four-element set.) Note that there is a corresponding action of $\mathbb{S}^{4}$ on the original moving frame signature $\mathcal{S}$, which is parametrized by the angular invariants, but this permutational 

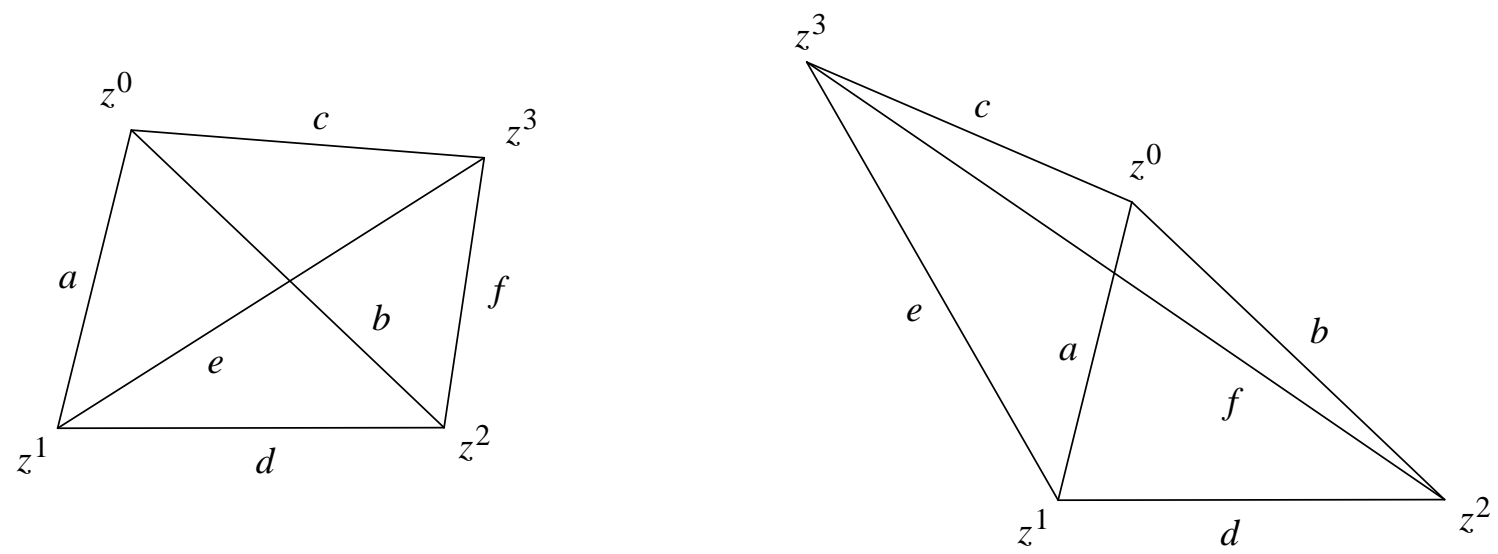

Figure 9. Four Point Euclidean Configurations.

symmetry is much less evident, since it relies on the complete system of angle and distance syzygies.

Another important remark is that one does not actually need the entire signature to reconstruct the curve. Let $\left(a_{0}, \ldots, f_{0}\right)$ be a point on $\widehat{\mathcal{S}}$. The "slice"

$$
\mathcal{S}_{0}=\left\{\left(a_{0}, b_{0}, c, d_{0}, e, f\right)\right\} \subset \widehat{\mathcal{S}}
$$

obtained by fixing the positions of $z^{0}, z^{1}, z^{2}$ and letting only $z^{3}$ vary will uniquely prescribe the position of $z^{3}$, and thus uniquely reconstruct the curve. Therefore, the entire signature is uniquely determined by a single slice $S_{0}$. Moreover, the final distance $f$ can take on only two possible values, and hence, based on its initial value $f_{0}$, is uniquely prescribed by continuity. As a consequence, the reconstruction of the curve effectively relies on only the two distances $c, e$ that parametrize the slice. Similar remarks hold in more general situations, and will be developed in detail in a subsequent paper devoted to the practical applications of these results in image processing.

Remark: The Cayley-Menger identity (1.3) is equivalent to the fundamental inner product syzygy $\operatorname{det}\left(\mathbf{v}_{i} \cdot \mathbf{v}_{j}\right)=0$ between three coplanar vectors $\mathbf{v}_{1}, \mathbf{v}_{2}, \mathbf{v}_{3}$, in accordance with Weyl's Second Main Theorem for the Euclidean group, [43; p. 75]. The syzygies among proper Euclidean invariants, which include an additional signed triangular area, can also be found in $[\mathbf{4 3} ;$ p. 77]. Incidentally, as with our earlier derivation of the Law of Cosines, the invariantization method can be used to effect a quick proof of such identities; details are left as an exercise for the interested reader.

Example 8.6. Equi-affine joint differential invariants. Consider next the special affine group $\mathrm{SA}(2)=\mathrm{SL}(2) \ltimes \mathbb{R}^{2}$ acting on planar curves as in (3.2). We consider $n$-point curves, with $z^{k}=z^{k}\left(t^{k}\right)$ parametrizing the $k^{\text {th }}$ point on the curve (or, more generally, a point on the $k^{\text {th }}$ curve). We first introduce a useful bracket notation

$$
\begin{aligned}
& {[i j]=\left[z^{i} z^{j}\right]=z^{i} \wedge z^{j}=\operatorname{det}\left(z^{i} z^{j}\right) \text {, }} \\
& {\left[\begin{array}{lll}
i j k
\end{array}\right]=\left[\begin{array}{lll}
z^{i} & z^{j} & z^{k}
\end{array}\right]=[i j]+[j k]+[k i]=\left(z^{j}-z^{i}\right) \wedge\left(z^{k}-z^{i}\right) .}
\end{aligned}
$$




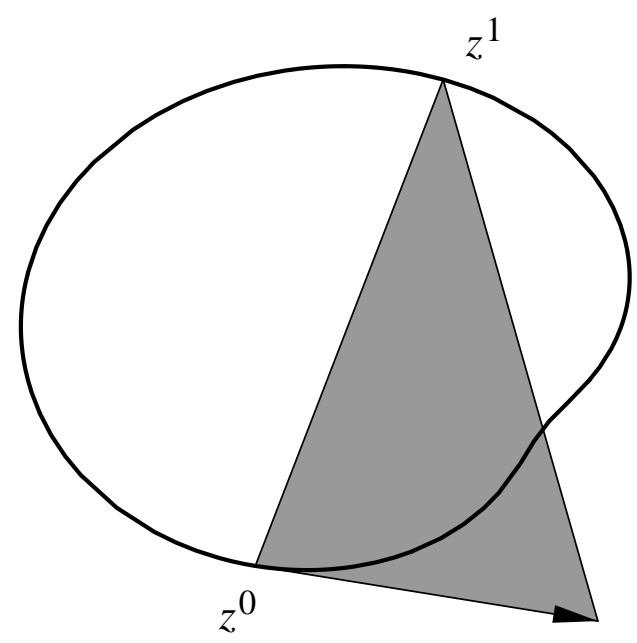

Figure 10. Tangent Triangle.

Note that

$$
\left[\begin{array}{lll}
i & j & k
\end{array}\right]=2 V(i, j, k)
$$

is twice the triangular area (3.6). Furthermore, we will indicate differentiation with respect to the parameters $t^{k}$ by a dot, so $\dot{z}^{k}=d z^{k} / d t^{k}$. Note that $d z^{k} / d t^{j}=0$ for $j \neq k$, so one needs to pay attention to which parameter is being differentiated when utilizing this notation. We also use dots over the bracket entries to indicate derivatives. In particular

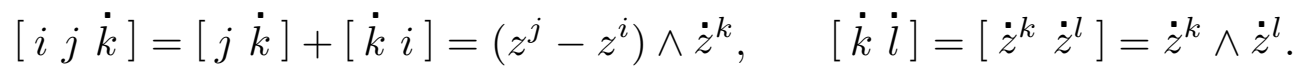

For example

$$
\frac{d}{d t^{k}}\left[\begin{array}{lll}
i & j & k
\end{array}\right]=\left[\begin{array}{lll}
i & j & \dot{k}
\end{array}\right], \quad \frac{d}{d t^{l}}\left[\begin{array}{lll}
i & l & k
\end{array}\right]=\left[\begin{array}{ll}
\dot{l} & \dot{k}
\end{array}\right]
$$

In particular, $V(0,1, \dot{0})=\frac{1}{2}\left[\begin{array}{lll}0 & 1 & 0\end{array}\right]$ is the area of the tangent triangle drawn in Figure 10. Note that this is not an equi-affine invariant - it is not even invariant under reparametrizations.

Let us first look at two-point joint differential invariants. We introduce the coordinate cross-section corresponding to the basic normalization

$$
y^{0}=v^{0}=0, \quad y^{1}=0, \quad v^{1}=1, \quad v_{y}^{0}=0 .
$$

Solving for the group parameters, and then inverting, leads to the first order left moving frame

$$
\widetilde{A}=\left(\frac{-\dot{z}^{0}}{\left[\begin{array}{lll}
0 & 1 & \dot{0}
\end{array}\right]}, \quad z^{1}-z^{0}\right), \quad \widetilde{d}=z^{0} .
$$


The invariant one-forms and associated differential operators are

$$
d y^{k} \longmapsto-\left[\begin{array}{lll}
0 & 1 & \dot{k}
\end{array}\right] d t^{k}, \quad D_{y^{k}} \longmapsto \mathcal{D}_{k}=-\frac{1}{\left[\begin{array}{lll}
0 & 1 & \dot{k}
\end{array}\right]} D_{t^{k}}
$$

(The minus sign is only important when we derive the recurrence formulae.) There is a single first order joint differential invariant:

$$
v_{y}^{1} \longmapsto I=\frac{\left[\begin{array}{ll}
\dot{0} & i
\end{array}\right]}{\left[\begin{array}{lll}
0 & 1 & \dot{0}
\end{array}\right]\left[\begin{array}{lll}
0 & 1 & i
\end{array}\right]}
$$

which, geometrically, is $\frac{1}{4}$ times the ratio of the area of the parallelogram spanned by the two tangent vectors over the product of the two tangent triangle areas. There are two second order invariants

$$
v_{y y}^{0} \longmapsto J_{0}=\frac{\left[\begin{array}{ll}
\dot{0} & \ddot{0}
\end{array}\right]}{\left[\begin{array}{lll}
0 & 1 & \dot{0}
\end{array}\right]^{3}}, \quad v_{y y}^{1} \longmapsto J_{1}=\frac{\left[\begin{array}{ll}
\dot{1} & \ddot{1}
\end{array}\right]}{\left[\begin{array}{lll}
0 & 1 & \dot{1}
\end{array}\right]^{3}} .
$$

We have already encountered the joint differential invariant $J_{0}$ in (8.6), and illustrated its geometry in Figure 7.

The recurrence formulae for the differentiated invariants are readily found via our infinitesimal algorithm, starting with the second order equi-affine Lie matrix

$$
\mathbf{L}_{2}=\left(\begin{array}{cccc}
1 & 0 & 0 & 0 \\
0 & 1 & 0 & 0 \\
-x & u & 2 u_{x} & 3 u_{x x} \\
u & 0 & -u_{x}^{2} & -3 u_{x} u_{x x} \\
0 & x & 1 & 0
\end{array}\right)
$$

The most important of these are

$$
\begin{array}{cll}
\mathcal{D}_{0} I=-J_{0}-I^{2}, & \mathcal{D}_{1} I=J_{1}-I^{2}, & \mathcal{D}_{0} J_{0}=K_{0}, \\
\mathcal{D}_{1} J_{0}=-3 I J_{0}, & \mathcal{D}_{0} J_{1}=-3 I J_{1}, & \mathcal{D}_{1} J_{1}=K_{1},
\end{array}
$$

where $v_{y y y}^{i} \mapsto K_{i}$ are the third order normalized invariants.

Theorem 8.7. Every two-point equi-affine joint differential invariant is a function of the fundamental first order invariant (8.23) and its derivatives with respect to the invariant differential operators $(8.22)$.

In the generic case, the joint signature of a two-point curve will be a two-dimensional surface, and, in view of the syzygies, one requires all three second order invariants $I, J_{0}, J_{1}$ to uniquely prescribe the reduced signature. There are no higher order product curves, so a one-dimensional signature implies the existence of a one-parameter equi-affine symmetry group for the product curve. The analysis is similar to the Euclidean case discussed above.

For three or more points, we normalize

$$
y^{0}=v^{0}=0, \quad y^{1}=0, \quad v^{1}=1, \quad v^{2}=0 .
$$




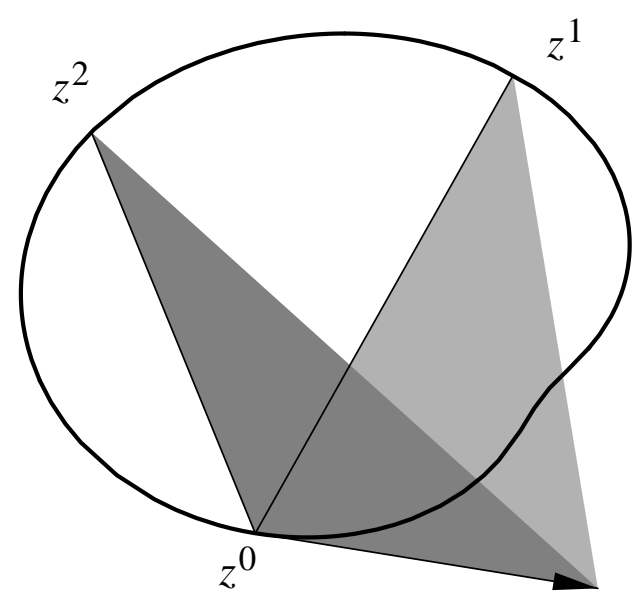

Figure 11. Triangular Areas.

The corresponding left moving frame is of order 0 , with

$$
\widetilde{A}=\left(\frac{z^{0}-z^{2}}{\left[\begin{array}{lll}
0 & 1 & 2
\end{array}\right]}, \quad z^{1}-z^{0}\right), \quad \widetilde{d}=z^{0} .
$$

The normalized values of

$$
y^{k} \longmapsto H_{k}=-\left[\begin{array}{lll}
0 & 1 & k
\end{array}\right], \quad v^{k} \longmapsto I_{k}=\frac{\left[\begin{array}{lll}
0 & k & 2
\end{array}\right]}{\left[\begin{array}{lll}
0 & 1 & 2
\end{array}\right]},
$$

provide the basic joint triangular area invariants, reproducing the results in Example 3.2. The invariant differential operators are

$$
\mathcal{D}_{k}=-\frac{1}{\left[\begin{array}{ll}
0 & 2 \dot{k}
\end{array}\right]} D_{t^{k}}=\frac{1}{\left(z^{0}-z^{2}\right) \wedge \dot{z}^{k}} D_{t^{k}}, \quad k=0,1,2
$$

The normalized first order joint differential invariants are

$$
v_{y}^{k} \longmapsto J_{k}=-\left[\begin{array}{lll}
0 & 1 & 2
\end{array}\right] \frac{\left[\begin{array}{lll}
0 & 1 & \dot{k}
\end{array}\right]}{\left[\begin{array}{lll}
0 & 2 & \dot{k}
\end{array}\right]}
$$

Note that the joint differential invariant $J_{0} /\left[\begin{array}{lll}0 & 1 & 2\end{array}\right]=\left[\begin{array}{lll}0 & 1 & 0\end{array}\right] /\left[\begin{array}{lll}0 & 2 & 0\end{array}\right]$ is the ratio of the two tangent triangular areas indicated in Figure 11. The key recurrence formulae

$$
\begin{aligned}
& \mathcal{D}_{0} H_{k}=I_{k}-H_{k} J_{0}-1, \quad \mathcal{D}_{1} H_{k}=H_{k} J_{1}-I_{k}, \quad \mathcal{D}_{k} H_{k}=1, \\
& \mathcal{D}_{0} I_{k}=\left(I_{k}-1+\frac{H_{k}}{H_{2}}\right) J_{0}, \quad \mathcal{D}_{1} I_{k}=-I_{1} J_{1}, \quad \mathcal{D}_{2} I_{k}=-\frac{H_{k} J_{2}}{H_{2}},
\end{aligned}
$$




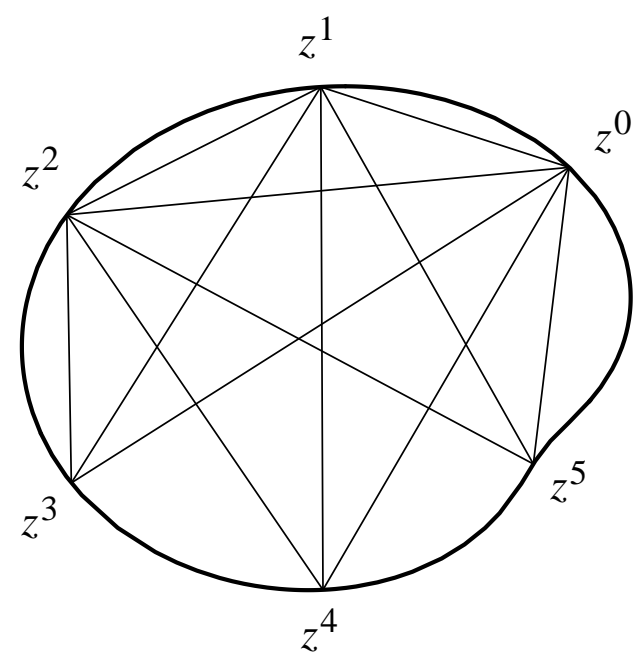

Figure 12. The Order Zero Equi-Affine Signature.

are derived using our infinitesimal algorithm. The identities for the derivatives of $H_{k}$ are valid for $k \geq 2$, whereas the formulae for the derivatives of $I_{k}$ require $k \geq 3$ to be valid since $I_{2}=0$. One can therefore generate the higher order differential invariants as derivatives of the $H_{k}, I_{k}$. In particular, for three-point equi-affine curves, we only require a single joint invariant - the triangular area $V(0,1,2)=\frac{1}{2}\left[\begin{array}{ll}0 & 1\end{array}\right]$ — to generate all others via invariant differentiation. This establishes the First Main Theorem for joint equi-affine differential invariants of planar curves.

Theorem 8.8. For $n \geq 3$, every $n$-point equi-affine joint differential invariant is a function of the fundamental area invariants $V(0,1, k), V(0,2, k), k=1, \ldots, n-1$, and their invariant derivatives using the differential operators (8.28).

In accordance with our general count, cf. Proposition 8.1, the signature curve for a one-point equi-affine curve has order 5. It is parametrized by the equi-affine curvature and its equi-affine arc-length derivative, $[\mathbf{1 8}, \mathbf{2 4}]$. A generic two-point equi-affine curve has second order signature surface. Three- and four- and five-point curves have first order equi-affine signatures. One must use $6=1+\operatorname{dim} \mathrm{SA}(2)$ distinct points on the curve to construct a zero ${ }^{\text {th }}$ order equi-affine signature. The minimal zero ${ }^{\text {th }}$ order signature will be parametrized by the seven area invariants $V(0,1, k), V(0,2, k)$ for $k=2,3,4,5$ indicated in Figure 12. As in the Euclidean case, there are permutation symmetries under $\mathbb{S}^{6}$ which are most easily manifested if one includes all 20 triangular areas based on the six points. However, here the syzygies are linear, and so their implementation on the minimal seven independent triangular areas is not as complicated as the Euclidean version.

Example 8.9. The most complicated planar example treated in the literature is the projective geometry of curves in the plane. The group is GL(3), or, rather, PSL(3), acting 
on $M=\mathbb{R P}^{2}$ or $\mathbb{C P}^{2}$ according to

$$
y=\frac{\alpha x+\beta u+\gamma}{\rho x+\sigma u+\tau}, \quad v=\frac{\lambda x+\mu u+\nu}{\rho x+\sigma u+\tau} .
$$

The ordinary projective curvature and arc length invariants can be found in $[\mathbf{1 5}, \mathbf{1 8}]$, and, for brevity, we will not repeat this computation here. We will, however, determine the projective joint differential invariants, which appears to be a new result. See $[\mathbf{5}, \mathbf{7}, \mathbf{1 1}]$ for a few particular examples and a discussion of applications in computer vision.

We begin with the easier case of $n$-point joint differential invariants when $n \geq 4$, where the moving frame has order 0. The joint invariants were already classified in Theorem 3.10. Unfortunately, the simple "infinite" normalizations adopted there are not so applicable to the prolonged joint action, since jets and infinitesimal generators at infinite points are not easy to handle. Therefore, we need to implement a set of computationally trickier finite normalizations before prolonging. The simplest finite normalizations

$$
y^{0}=v^{0}=0, \quad y^{1}=1, \quad v^{1}=0, \quad y^{2}=0, \quad v^{2}=1, \quad y^{3}=v^{3}=1 .
$$

lead, after some algebra, to the explicit moving frame formulae

$$
\begin{aligned}
& \alpha=-\xi\left(u^{2}-u^{0}\right), \quad \beta=\xi\left(x^{2}-x^{0}\right), \quad \gamma=-\alpha x^{0}-\beta u^{0}=\xi\left[\begin{array}{ll}
0 & 2
\end{array}\right], \\
& \lambda=-\eta\left(u^{1}-u^{0}\right), \quad \quad \mu=\eta\left(x^{1}-x^{0}\right), \quad \nu=-\lambda x^{0}-\mu u^{0}=\eta\left[\begin{array}{ll}
0 & 1
\end{array}\right] \text {, } \\
& \rho=-\zeta\left(u^{2}-u^{1}\right)-(\xi+\eta)\left(u^{1}-u^{0}\right), \quad \sigma=\zeta\left(x^{2}-x^{1}\right)+(\xi+\eta)\left(x^{1}-x^{0}\right) \text {, } \\
& \tau=-\rho x^{1}-\sigma u^{1}+\alpha\left(x^{1}-x^{0}\right)+\beta\left(u^{1}-u^{0}\right)=\eta\left[\begin{array}{ll}
0 & 1
\end{array}\right]+\xi\left[\begin{array}{ll}
0 & 2
\end{array}\right]+(\zeta-\xi)\left[\begin{array}{ll}
1 & 2
\end{array}\right],
\end{aligned}
$$

where, using our bracket notation (8.20),

$$
\begin{gathered}
\xi=-\theta\left[\begin{array}{lll}
0 & 1 & 3
\end{array}\right]\left[\begin{array}{lll}
1 & 2 & 3
\end{array}\right], \quad \eta=-\theta\left[\begin{array}{lll}
0 & 2 & 3
\end{array}\right]\left[\begin{array}{lll}
1 & 2 & 3
\end{array}\right], \\
\zeta=\theta\left[\begin{array}{lll}
0 & 1 & 3
\end{array}\right]\left(\left[\begin{array}{lll}
0 & 1 & 3
\end{array}\right]-\left[\begin{array}{lll}
0 & 1 & 2
\end{array}\right]\right) .
\end{gathered}
$$

Here the group parameter $\theta$ remains unspecified, although, if desired, the unimodularity constraint can be used to fix its value. One finds that the remaining variables reduce to the joint invariants

$$
\begin{aligned}
y^{k} & \longmapsto \quad I_{k}=\frac{1}{1+C(0 ; 1, k, 3,2)-C(2 ; 0,3, k, 1)}, \quad k \geq 4, \\
v^{k} & \longmapsto \quad J_{k}=\frac{1}{1+C(0 ; 1,3, k, 2)-C(1 ; 0,3, k, 2)},
\end{aligned}
$$

depending on the same volume cross ratios (3.23) derived earlier via the "infinite" normalizations. One can recover the two independent cross-ratios as functions of $I_{k}, J_{k}$ in view of the syzygies

$$
C(0 ; 1,3, k, 2)=\frac{1}{C(0 ; 1, k, 3,2)}, \quad C(1 ; 0,3, k, 2)=\frac{C(2 ; 0,3, k, 1)}{C(0 ; 1, k, 3,2)} .
$$

The projectively invariant differential operators are

$$
D_{y^{k}} \longmapsto \mathcal{D}_{k}=-\frac{A_{k}}{\left[\begin{array}{lll}
0 & 1 & 3
\end{array}\right]\left[\begin{array}{lll}
0 & 2 & 3
\end{array}\right]\left[\begin{array}{lll}
1 & 2 & 3
\end{array}\right] B_{k}} D_{t^{k}}
$$


where

$$
\begin{aligned}
& A_{k}=\left(\left[\begin{array}{lll}
0 & 1 & 3
\end{array}\right]\left[\begin{array}{lll}
0 & 2 & 3
\end{array}\right]\left[\begin{array}{lll}
1 & 2 & k
\end{array}\right]-\left[\begin{array}{lll}
0 & 1 & 3
\end{array}\right]\left[\begin{array}{lll}
1 & 2 & 3
\end{array}\right]\left[\begin{array}{lll}
0 & 2 & k
\end{array}\right]-\left[\begin{array}{lll}
0 & 2 & 3
\end{array}\right]\left[\begin{array}{lll}
1 & 2 & 3
\end{array}\right]\left[\begin{array}{lll}
0 & 1 & k
\end{array}\right]\right)^{2}, \\
& B_{k}=\left(\left[\begin{array}{lll}
0 & 1 & 3
\end{array}\right]\left[\begin{array}{lll}
1 & 2 & k
\end{array}\right]-\left[\begin{array}{lll}
1 & 2 & 3
\end{array}\right]\left[\begin{array}{lll}
0 & 1 & k
\end{array}\right]\right)\left[\begin{array}{lll}
0 & 2 & \dot{k}
\end{array}\right]- \\
& -\left(\left[\begin{array}{lll}
0 & 1 & 3
\end{array}\right]\left[\begin{array}{lll}
1 & 2 & \dot{k}
\end{array}\right]-\left[\begin{array}{lll}
1 & 2 & 3
\end{array}\right]\left[\begin{array}{lll}
0 & 1 & \dot{k}
\end{array}\right]\right)\left[\begin{array}{lll}
0 & 2 & k
\end{array}\right] \text {. }
\end{aligned}
$$

The recurrence formulae for the differentiated invariants are determined by the same infinitesimal algorithm, starting with the second order projective Lie matrix

$$
\mathbf{L}_{2}=\left(\begin{array}{cccc}
1 & 0 & 0 & 0 \\
0 & 1 & 0 & 0 \\
x & 0 & -u_{x} & -2 u_{x x} \\
u & 0 & -u_{x}^{2} & -3 u_{x} u_{x x} \\
0 & x & 1 & 0 \\
0 & u & u_{x} & u_{x x} \\
x^{2} & x u & u-x u_{x} & -3 x u_{x x} \\
x u & u^{2} & -u_{x}^{2} & -3 x u_{x} u_{x x}
\end{array}\right) .
$$

Let $v_{y}^{k} \mapsto K_{k}$ denote the normalized first order differential invariants — see (8.39) below. The nontrivial recurrence formulae are

$$
\begin{aligned}
& \mathcal{D}_{0} I_{k}=-I_{k}\left(I_{k}-1\right) K_{0}-\left(I_{k}-1\right)\left(J_{k}-1\right), \quad \mathcal{D}_{1} I_{k}=I_{k}\left(I_{k}-1\right) K_{1}+I_{k}\left(J_{k}-1\right), \\
& \mathcal{D}_{2} I_{k}=I_{k}\left(I_{k}-1\right) K_{2}+\left(I_{k}-1\right) J_{k}, \quad \mathcal{D}_{3} I_{k}=-I_{k}\left(I_{k}-1\right) K_{3}-I_{k} J_{k}, \quad \mathcal{D}_{k} I_{k}=1, \\
& \mathcal{D}_{0} J_{k}=-\left(I_{k}-1\right)\left(J_{k}-1\right) K_{0}-J_{k}\left(J_{k}-1\right), \quad \mathcal{D}_{1} J_{k}=-I_{k}\left(J_{k}-1\right) K_{1}+J_{k}\left(J_{k}-1\right), \\
& \mathcal{D}_{2} J_{k}=-\left(I_{k}-1\right) J_{k} K_{2}+J_{k}\left(J_{k}-1\right), \quad \mathcal{D}_{3} J_{k}=-I_{k} J_{k} K_{3}-J_{k}\left(J_{k}-1\right), \quad \mathcal{D}_{k} J_{k}=K_{k},
\end{aligned}
$$

and all other derivatives are zero on product curves. Therefore, on the open subset of the product jet space where

$$
I_{k}\left(I_{k}-1\right) J_{k}\left(J_{k}-1\right) \neq 0, \quad k=0,1, \ldots, n,
$$

all the first (and higher) order differential invariants are generated from the joint invariants by invariant differentiation! We have thus established a First Main Theorem for joint projective differential invariants.

Theorem 8.10. For $n \geq 5$, every $n$-point joint projective differential invariant is a function of the fundamental cross-ratio invariants $C(0 ; 1,3, k, 2), C(1 ; 0,3, k, 2), k=$ $4, \ldots, n-1$, and their derivatives with respect to the invariant differential operators (8.35).

There are no four-point joint invariants, and so when $n=4$ we can still use the same order 0 moving frame, but must construct the first order differential invariants directly. We substitute the moving frame expressions (8.33), (8.34) into the formulae

$$
v_{y}=\frac{\left(\lambda x_{t}+\mu u_{t}\right)(\rho x+\sigma u+\tau)-\left(\rho x_{t}+\sigma u_{t}\right)(\lambda x+\mu u+\nu)}{\left(\alpha x_{t}+\beta u_{t}\right)(\rho x+\sigma u+\tau)-\left(\rho x_{t}+\sigma u_{t}\right)(\alpha x+\beta u+\gamma)}
$$


for the first prolongation of (8.31). The result is that the derivatives $v_{y}^{0}, v_{y}^{1}, v_{y}^{2}, v_{y}^{3}$ reduce to the first order differential invariants

$$
\begin{array}{ll}
K_{0}=C(0 ; 3,2,1, \dot{0}), & K_{2}=C(2 ; 3,0,2, \dot{0})-1, \\
K_{1}=\frac{1}{C(1 ; 3,0,2, \dot{1})-1}, & K_{3}=\frac{C(2 ; 3,1,0, \dot{3})-1}{C(1 ; 3,1,0, \dot{3})-1},
\end{array}
$$

where

$$
C(i ; j, k, l, \dot{m})=\frac{\left[\begin{array}{lll}
i & j & k
\end{array}\right]\left[\begin{array}{lll}
i & l & \dot{m}
\end{array}\right]}{\left[\begin{array}{lll}
i & j & l
\end{array}\right]\left[\begin{array}{lll}
i & k & \dot{m}
\end{array}\right]}
$$

is a "derived" area cross-ratio, which, interestingly, is a particular ratio of equi-affine joint differential invariants (8.29). An infinitesimal analysis of the resulting recurrence formulae proves that all higher order differential invariants are obtained by invariant differentiation.

Theorem 8.11. Every 4-point joint projective differential invariant is a function of the fundamental derived cross-ratio invariants (8.40) and their derivatives with respect to the invariant differential operators (8.35).

For the 3-point joint differential invariants, we may begin with the order 0 normalizations

$$
y^{0}=v^{0}=0, \quad y^{1}=1, \quad v^{1}=0, \quad y^{2}=0, \quad v^{2}=1 .
$$

This leads to the same moving frame formulae (8.33) as before, but now $\xi, \eta, \zeta$ are as yet unspecified group parameters, that must be normalized using the first order derivatives. Substituting (8.33) into the prolongation formulae (8.38), we find that we can normalize $v_{y}^{0}=1$ and $v_{y}^{1}=1$.

Caution: The more "natural" normalization $v_{y}^{1}=-1$ is, in fact, not allowed and does not define a cross-section. Indeed, this would require $\dot{z}^{1}$ to be parallel to $z^{2}-z^{1}$, which is clearly not a generic condition on the curve.

After some algebra, the result is

$$
\begin{gathered}
\xi=\theta\left[\begin{array}{lll}
0 & 1 & \dot{0}
\end{array}\right]\left[\begin{array}{lll}
1 & 2 & i
\end{array}\right], \quad \eta=\theta\left[\begin{array}{lll}
0 & 2 & 0
\end{array}\right]\left[\begin{array}{lll}
1 & 2 & i
\end{array}\right], \\
\zeta=\theta\left(\left[\begin{array}{lll}
0 & 1 & 0
\end{array}\right]\left[\begin{array}{lll}
1 & 2 & i
\end{array}\right]-2\left[\begin{array}{lll}
0 & 2 & 0
\end{array}\right]\left[\begin{array}{lll}
0 & 1 & i
\end{array}\right]\right),
\end{gathered}
$$

which takes the place of $(8.34)$. The remaining jet coordinate then produces the first order 3 -point projective joint differential invariant: $v_{y}^{2} \longmapsto 2 I-1$, where

$$
I=\frac{\left[\begin{array}{lll}
0 & 2 & \dot{0}
\end{array}\right]\left[\begin{array}{lll}
0 & 1 & \dot{1}
\end{array}\right]\left[\begin{array}{lll}
1 & 2 & \dot{2}
\end{array}\right]}{\left[\begin{array}{lll}
0 & 1 & \dot{0}
\end{array}\right]\left[\begin{array}{lll}
1 & 2 & \dot{1}
\end{array}\right]\left[\begin{array}{lll}
0 & 2 & \dot{2}
\end{array}\right]}
$$

Referring to Figure 13, we see that $I$ is the ratio of the product of the three triangular areas in the first diagram over the product of the three triangular areas in the second diagram, and hence has a very pretty geometrical formulation. It is, of course, the unique ratio of the four-point joint differential invariants (8.40) that does not depend on the fourth point $z^{3}$. Another application of our infinitesimal algorithm for the recurrence formulae completes the proof of the following result. 

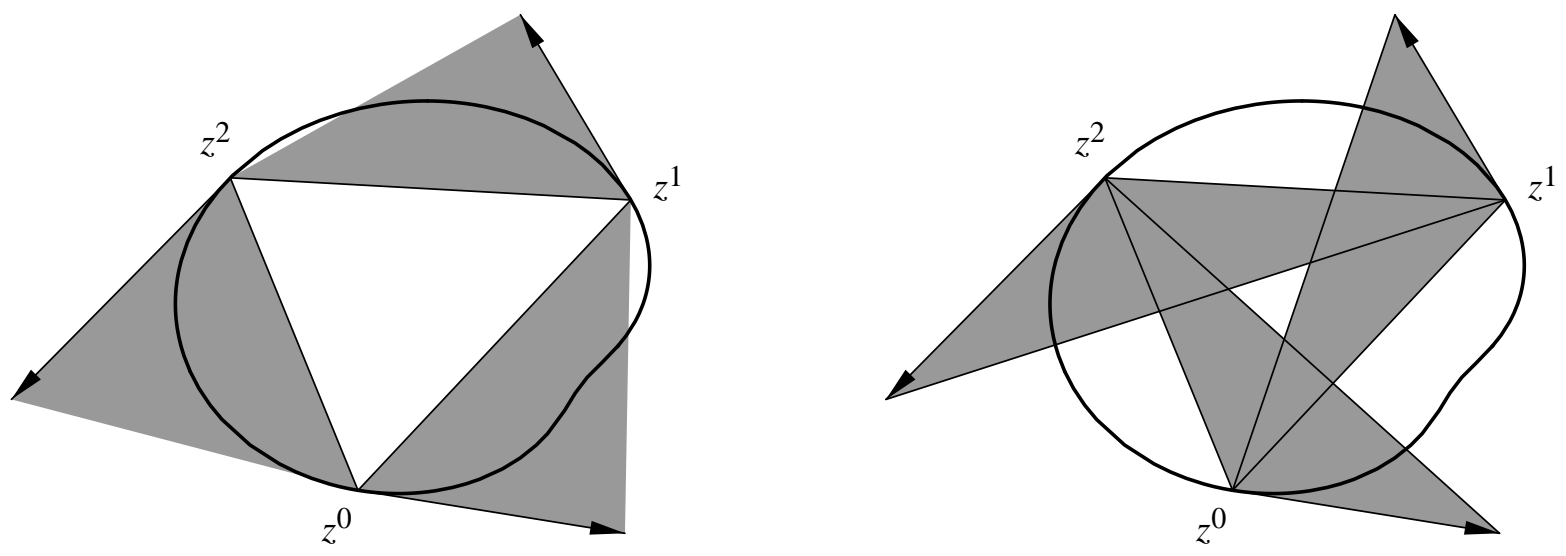

Figure 13. First Order Projective Joint Differential Invariant.

Theorem 8.12. Every 3-point joint projective differential invariant is a function of the fundamental first order joint differential invariant (8.42) and its invariant derivatives.

The case of two-point projective joint differential invariants has some interesting twists in the computation, which we now summarize. It begins with the order zero normalizations $y^{0}=v^{0}=v^{1}=0, y^{1}=1$. The first order normalizations are $v_{y}^{0}=v_{y}^{1}=1$. (This is possible, since the condition that the tangents at two distinct points be parallel is not projectively invariant.) This yields the preliminary normalizations

$$
\begin{aligned}
\alpha x+\beta u+\gamma & =\xi\left[\begin{array}{lll}
0 & z & 0
\end{array}\right]+\eta\left[\begin{array}{lll}
0 & 1 & z
\end{array}\right], \\
\lambda x+\mu u+\nu & =\eta\left[\begin{array}{lll}
0 & 1 & z
\end{array}\right] \\
\rho x+\sigma u+\tau & =\xi\left[\begin{array}{lll}
0 & z & 0
\end{array}\right]+\zeta\left[\begin{array}{lll}
1 & z & 1
\end{array}\right],
\end{aligned}
$$

where $\xi, \eta, \zeta$ remain to be normalized. Substituting (8.43) into the second order prolongation formulae yields

$$
v_{y y}^{0} \longmapsto-\frac{\xi \zeta}{\eta^{2}} \frac{\left[\begin{array}{lll}
0 & 1 & \dot{1}
\end{array}\right]\left[\begin{array}{lll}
\dot{0} & \ddot{0}
\end{array}\right]}{\left[\begin{array}{lll}
0 & 1 & \dot{0}
\end{array}\right]^{2}}, \quad v_{y y}^{1} \longmapsto-\frac{\xi \zeta}{\eta^{2}} \frac{\left[\begin{array}{lll}
0 & 1 & 0
\end{array}\right]\left[\begin{array}{lll}
\dot{1} & \ddot{1}
\end{array}\right]}{\left[\begin{array}{lll}
0 & 1 & i
\end{array}\right]^{2}}
$$

Therefore, the ratio of these two quantities

$$
I=\frac{\left[\begin{array}{lll}
0 & 1 & \dot{0}
\end{array}\right]^{3}\left[\begin{array}{ll}
\dot{1} & \ddot{1}
\end{array}\right]}{\left[\begin{array}{lll}
0 & 1 & \dot{1}
\end{array}\right]^{3}\left[\begin{array}{lll}
\dot{0} & \ddot{0}
\end{array}\right]}
$$

is a second order differential invariant. Note that $I$ is the ratio of the two second order joint equi-affine invariants (8.24); see Figure 7. The existence of a second order joint projective differential invariant is unexpected, and reflects that fact that PSL(3) does not act freely on $\left(\mathrm{J}^{2}\right)^{\times 2}$, even though the space has the same dimension, namely 8, as the group. As a consequence, we can only normalize one of the remaining group parameters at second order, and the final moving frame is third order. There are several different choices for the 
remaining normalizations; the simplest (albeit asymmetric) choice, is to set

$$
v_{y y}^{0}=-1, \quad v_{y y y}^{0}=0 .
$$

These imply that

$$
\xi=-\frac{\left[\begin{array}{lll}
0 & 1 & 0
\end{array}\right]^{2}}{3\left[\begin{array}{lll}
0 & 1 & \dot{1}
\end{array}\right]\left[\begin{array}{lll}
\dot{0} & \ddot{0}
\end{array}\right]} P^{2} \zeta, \quad \eta=-\frac{1}{3} P \zeta,
$$

where we assume the points are configured so that

$$
0 \neq P=\frac{\left[\begin{array}{lll}
\dot{0} & \ddot{0}
\end{array}\right]\left[\begin{array}{lll}
0 & 1 & 0
\end{array}\right]\left[\begin{array}{lll}
0 & 1 & i
\end{array}\right]+3\left[\begin{array}{ll}
\dot{0} & \ddot{0}
\end{array}\right]\left(\left[\begin{array}{lll}
0 & 1 & \dot{0}
\end{array}\right]\left[\begin{array}{ll}
\dot{0} & \dot{1}
\end{array}\right]+\left[\begin{array}{lll}
0 & 1 & 0
\end{array}\right]\left[\begin{array}{lll}
0 & 1 & i
\end{array}\right]\right)}{\left[\begin{array}{lll}
0 & 1 & \dot{0}
\end{array}\right]^{2}\left[\begin{array}{lll}
\dot{0} & \ddot{0}
\end{array}\right]} .
$$

The final parameter $\zeta$ can be normalized by imposing unimodularity, but, as before, this is unnecessary. The invariant differential operators are

$$
\mathcal{D}_{0}=\frac{3\left[\begin{array}{lll}
0 & 1 & i
\end{array}\right]}{\left[\begin{array}{lll}
0 & 1 & 0
\end{array}\right] P} D_{t^{0}}, \quad \mathcal{D}_{1}=-\frac{3\left[\begin{array}{lll}
0 & 1 & 0
\end{array}\right]^{3} P^{2}}{\left[\begin{array}{lll}
0 & 1 & \dot{1}
\end{array}\right]^{2}\left[\begin{array}{lll}
\dot{0} & \ddot{0}
\end{array}\right]} D_{t^{1}} .
$$

(One can, by imposing a more symmetric normalization, obtain symmetrical formulae, but these are sufficient for our purposes.) The recurrence formulae are found by our usual infinitesimal method:

$$
\begin{array}{llrl}
\mathcal{D}_{0} I & =-3 I, & \mathcal{D}_{1} I=J-3 I^{2}, \\
\mathcal{D}_{0} J=-\frac{1}{3}\left(J-3 I^{2}\right) K_{0}-6 J+3 I^{2}-3 I, & \mathcal{D}_{1} J=K_{1}-(5 I-1) J-9 I^{2},
\end{array}
$$

where $v_{y y y}^{2} \mapsto J, v_{y y y y}^{i} \mapsto K_{i}$ are the normalized third and fourth order invariants. These prove that, on the open subset of $\left(\mathrm{J}^{n}\right)^{\times 2}$ where $\mathcal{D}_{1} I \neq 0$, all higher order differential invariants are obtained by differentiation.

Theorem 8.13. Every 2-point joint projective differential invariant is a function of the fundamental second order joint differential invariant (8.44) and its invariant derivatives.

In accordance with our general count, cf. Proposition 8.1, the smallest number of points that will produce an order zero signature is $9=1+\operatorname{dim} \operatorname{PSL}(3)$, parametrized by a total of $18-8=10$ different joint invariant area cross-ratios. Generically, a first order signature curve can be constructed with 5 points using $15-8=7$ first order joint differential invariants. Three and four points require a second order projective signature curve, two points necessitate going to joint differential invariants of order 4, while the usual one-point projective signature curve has order 8 , but only requires two differential invariants - the projective curvature and its projective arc-length derivative, cf. [15, 18].

\section{Joint Differential Invariants for Space Curves and Surfaces.}

As we know, the moving frame methods are not tied to just planar curves, but are equally applicable to curves and surfaces in three-dimensional space. For simplicity, we just consider the Euclidean and equi-affine cases here, leaving out the construction of joint 
differential invariants for projective space curves and surfaces. For simplicity, we only consider generic situations, avoiding particular geometric configurations that require higher order, more complicated normalizations. The complicated infinitesimal syzygy computations are suppressed for brevity. They were implemented using a general MATHEMATICA routine; details are available upon request from the author.

Example 9.1. We begin by computing the joint differential invariants for $n$-point space curves $C \subset \mathbb{R}^{3}$ under the Euclidean groups $\mathrm{SE}(3)$ and $\mathrm{E}(3)$. Applications in computer vision and invariant numerical algorithms can be found in $[\mathbf{3 8}, \mathbf{3}]$. We shall use the notation $z=(x, u, \widetilde{u})$ for points in $\mathbb{R}^{3}$, and $w=(y, v, \widetilde{v})=R z+d$ for their images under a Euclidean transformation. We view $x, y$ as the independent variables and $u, \widetilde{u}, v, \widetilde{v}$ as dependent variables.

We already treated, in Example 3.6, the case when there are $n \geq 3$ points. The moving frame (3.14) leads to the zero ${ }^{\text {th }}$ order joint distance invariants $\left\|z^{i}-z^{j}\right\|$ along with the oriented simplex volume $V(0,1,2,3)$ in the case of $\operatorname{SE}(3)$. The invariant differential operators are

$$
D_{y^{k}} \quad \longmapsto \quad \mathcal{D}_{k}=\frac{1}{\left(z^{1}-z^{0}\right) \cdot z_{t}^{k}} D_{t^{k}}
$$

It is not hard to prove, either directly or using the infinitesimal algorithm, that all first and higher order differential invariants are obtained from the joint distance (and volume) invariants by invariant differentiation. In the three point case, one must actually differentiate twice to recover some of the first order joint differential invariants.

For a general curve, there are $3 n-6$ functionally independent $n$-point joint Euclidean invariants. Therefore, one requires $3 n-6>n$, or $n \geq 4$ points in order to achieve at least one zero ${ }^{\text {th }}$ order syzygy among the distance invariants, and hence a zero ${ }^{\text {th }}$ order joint signature. It is interesting that this is the same number of points as was required in the planar case. However, in the three-dimensional case, there are no universal syzygies among the six interpoint distances among four points in $\mathbb{R}^{3}$, and so the two distance syzygies are both dependent upon the space curve. In particular, the curve is planar if and only if it satisfies the Cayley-Menger syzygy (1.3).

In the case of $n=2$ point curves, the six normalizations

$$
w^{0}=0, \quad w^{1}=\lambda e_{1}, \quad \widetilde{v}_{y}^{0}=0,
$$

will suffice to prescribe the moving frame up to one or two sign ambiguities. The translation component of the resulting left moving frame is $\widetilde{d}=z^{0}$, while the rotation matrix becomes

$$
\widetilde{R}=\left(\frac{z^{1}-z^{0}}{\left\|z^{1}-z^{0}\right\|}, \sigma \varepsilon \frac{\left[\left(z^{1}-z^{0}\right) \wedge z_{t}^{0}\right] \wedge\left(z^{1}-z^{0}\right)}{\left\|\left(z^{1}-z^{0}\right) \wedge z_{t}^{0}\right\|}\left\|z^{1}-z^{0}\right\|, \varepsilon \frac{\left(z^{1}-z^{0}\right) \wedge z_{t}^{0}}{\left\|\left(z^{1}-z^{0}\right) \wedge z_{t}^{0}\right\|}\right) .
$$

Here $\sigma, \varepsilon= \pm 1$ are as yet unspecified signs, with $\sigma=+1$ in the case of $\operatorname{SE}(3)$. The other derivative coordinate reduces to

$$
v_{y}^{0} \longmapsto \sigma \varepsilon \frac{\left\|\left(z^{1}-z^{0}\right) \wedge z_{t}^{0}\right\|}{\left(z^{1}-z^{0}\right) \cdot z_{t}^{0}}=\sigma \varepsilon \frac{\left|\sin \phi^{0}\right|}{\cos \phi^{0}}
$$


where $\phi^{k}$ denotes the angle between the chord $z^{1}-z^{0}$ and the tangent to the curve at $z^{k}$, which is only determined up to sign and integer multiples of $\pi$. We require $v_{y}^{0}>0$, which implies

$$
\sigma \varepsilon=\operatorname{sgn}\left(\cos \phi^{0}\right)
$$

This leads to the first order joint differential invariant

$$
v_{y}^{0} \longmapsto\left|\tan \phi^{0}\right| \text {. }
$$

In the case of $\operatorname{SE}(2)$, where $\sigma=+1$, equations (9.2), (9.3) uniquely prescribe the moving frame. The other two first order derivatives now reduce to

$$
v_{y}^{1} \longmapsto \frac{\operatorname{sgn}\left(\cos \phi^{0}\right) \mathbf{t}^{0} \cdot \mathbf{t}^{1}}{\cos \phi^{1}\left|\sin \phi^{0}\right|}-\left|\cot \phi^{0}\right|, \quad \widetilde{v}_{y}^{1} \longmapsto \varepsilon \frac{\left(z^{1}-z^{0}\right) \cdot\left(\mathbf{t}^{0} \wedge \mathbf{t}^{1}\right)}{\left|\sin \phi^{0}\right| \cos \phi^{1}\left\|z^{1}-z^{0}\right\|} .
$$

We use

$$
\mathbf{t}^{k}=\frac{z_{t}^{k}}{\left\|z_{t}^{k}\right\|}, \quad k=0,1
$$

to denote the unit tangent vectors for the given parametrization. Although these are not invariant under reversal of parametrization, the distinguished unit tangent vectors

$$
\widehat{\mathbf{t}}^{k}=\operatorname{sgn}\left(\cos \phi^{k}\right) \mathbf{t}^{k}=\operatorname{sgn}\left(\cos \phi^{k}\right) \frac{z_{t}^{k}}{\left\|z_{t}^{k}\right\|}, \quad k=0,1,
$$

are invariantly defined - by the requirement $0 \leq \Varangle\left(z^{1}-z^{0}, \widehat{\mathbf{t}}^{k}\right)<\frac{1}{2} \pi$. Therefore,

$$
v_{y}^{1} \longmapsto \frac{\widehat{\mathbf{t}}^{0} \cdot \widehat{\mathbf{t}}^{1}}{\left|\sin \phi^{0}\right|\left|\cos \phi^{1}\right|}-\left|\cot \phi^{0}\right|, \quad \widetilde{v}_{y}^{1} \longmapsto \sigma \frac{\left(z^{1}-z^{0}\right) \cdot\left(\widehat{\mathbf{t}}^{0} \wedge \widehat{\mathbf{t}}^{1}\right)}{\left|\sin \phi^{0}\right|\left|\cos \phi^{1}\right|\left\|z^{1}-z^{0}\right\|} .
$$

In the orientation-preserving case of $\mathrm{SE}(3), \sigma=+1$, and so the three fundamental first order joint differential invariants are $(9.4),(9.6)$. In the case of $\mathrm{E}(3)$, we can fix

$$
\sigma=\operatorname{sgn}\left[\left(z^{1}-z^{0}\right) \cdot\left(\widehat{\mathbf{t}}^{0} \wedge \widehat{\mathbf{t}}^{1}\right)\right]
$$

by the orientation determined by the chord connecting the two points and the two distinguished tangent directions. The absolute value of the volume of the associated simplex, which is a function of the previous joint differential invariants, is invariant. Note that the invariant one-forms are

$$
d y^{k} \longmapsto \frac{\left(z^{1}-z^{0}\right) \cdot z_{t}^{k}}{\left\|z^{1}-z^{0}\right\|} d t^{k}=\cos \phi^{k} d s^{k}
$$

where $d s^{k}=\left\|z_{t}^{k}\right\| d t^{k}$ denotes the usual arc length form, which is not invariant under a reversal of the parametrization. The invariant differential operators are

$$
D_{y^{k}} \longmapsto \mathcal{D}_{k}=\frac{\left\|z^{1}-z^{0}\right\|}{\left(z^{1}-z^{0}\right) \cdot z_{t}^{k}} D_{t^{k}}=\frac{1}{\cos \phi^{k}} D_{s^{k}} .
$$

An infinitesimal analysis of the recurrence formulae produces the First Main Theorem for two-point Euclidean space curves. 
Theorem 9.2. All two-point differential invariants for Euclidean space curves are obtained by invariant differentiation of the first order invariants

$$
\left\|z^{1}-z^{0}\right\|, \quad\left|\cos \phi^{0}\right|, \quad\left|\cos \phi^{1}\right|, \quad \widehat{\mathbf{t}}^{0} \cdot \widehat{\mathbf{t}}^{1}=\cos \psi
$$

and, in the orientation-preserving case, the simplex volume

$$
\frac{1}{6}\left(z^{1}-z^{0}\right) \cdot\left(\widehat{\mathbf{t}}^{0} \wedge \widehat{\mathbf{t}}^{1}\right) .
$$

Here $\widehat{\mathbf{t}}^{k}$ are the distinguished unit tangent vectors $(9.5), \psi=\Varangle\left(\widehat{\mathbf{t}}^{0}, \widehat{\mathbf{t}}^{1}\right)$ is the angle between them, and $\phi^{k}=\Varangle\left(z^{1}-z^{0}, z_{t}^{k}\right)$.

Remark: Here is a case where the joint invariant $I=\left\|z^{1}-z^{0}\right\|$ does not generate all the joint differential invariants. Of the two first order joint differential invariants in (9.6), the second equals the invariant derivative $\mathcal{D}_{1} I$ of the distance invariant, while the first cannot be obtained by differentiation. These facts follow immediately from the recurrence formulae, which, for brevity, we omit.

Finally, in view of our experience with planar curves, it is worth briefly repeating the analysis for 1-point space curves. The initial normalizations

$$
w=0, \quad v_{y}=\widetilde{v}_{y}=0, \quad \widetilde{v}_{y y}=0,
$$

produce a left moving frame with translation component $\widetilde{d}=z$, and rotation matrix

$$
\widetilde{R}=\left(\delta \frac{z_{t}}{\left\|z_{t}\right\|}, \varepsilon \frac{\left(z_{t} \wedge z_{t t}\right) \wedge z_{t}}{\left\|z_{t}\right\|\left\|z_{t} \wedge z_{t t}\right\|}, \sigma \frac{z_{t} \wedge z_{t t}}{\left\|z_{t} \wedge z_{t t}\right\|}\right)
$$

Here $\delta, \varepsilon, \sigma= \pm 1$, with product $\delta \varepsilon \sigma=+1$ in the case of $\operatorname{SE}(3)$. The unnormalized second and third order derivatives become

$$
\begin{aligned}
& v_{y y} \longmapsto \varepsilon \frac{\left\|z_{t} \wedge z_{t t}\right\|}{\left\|z_{t}\right\|^{3}}, \\
& v_{y y y} \longmapsto \delta \varepsilon \frac{\left\|z_{t}\right\|^{2}\left(z_{t} \wedge z_{t t}\right) \cdot\left(z_{t} \wedge z_{t t t}\right)-3\left\|z_{t} \wedge z_{t t}\right\|^{2}\left(z_{t} \cdot z_{t t}\right)}{\left\|z_{t}\right\|^{6}\left\|z_{t} \wedge z_{t t}\right\|^{2}}, \\
& \widetilde{v}_{y y y} \longmapsto \delta \sigma \frac{\left(z_{t} \wedge z_{t t}\right) \cdot z_{t t t}}{\left\|z_{t}\right\|^{3}\left\|z_{t} \wedge z_{t t}\right\|} .
\end{aligned}
$$

We normalize $\varepsilon=+1$ to make $v_{y y}>0$, and so

$$
v_{y y} \longmapsto \quad \kappa=\frac{\left\|z_{t} \wedge z_{t t}\right\|}{\left\|z_{t}\right\|^{3}} \neq 0
$$

reduces to the usual Euclidean curvature invariant, which has been assumed to be non-zero in order to perform the indicated normalizations. If we introduce the standard oriented arc length element $d s=\left\|z_{t}\right\| d t$, then $v_{y y y} \mapsto \delta \kappa_{s}$, and so we can require $v_{y y y}>0$ by normalizing

$$
\delta=\operatorname{sgn} \kappa_{s}, \quad \text { so that } \quad v_{y y y} \mapsto\left|\kappa_{s}\right| .
$$


Therefore, we should parametrize the curve in the invariant manner to ensure that $\kappa_{s}>0$. Finally, in the case of $\mathrm{SE}(3)$, we have $\delta \sigma=\varepsilon=+1$, and so $\widetilde{v}_{y y y} \mapsto \tau \kappa$ reduces to the product of the standard torsion and curvature invariants, [24], whose sign determines the "handedness" or orientation of the curve. In the case of $\mathrm{E}(3)$, we set $\sigma=\operatorname{sgn}\left(\tau \kappa_{s}\right)$, and then only the absolute value of torsion, $|\tau|$, is invariant. Points where $\tau \kappa \kappa_{s}=0$ require a higher order moving frame to resolve the sign ambiguities.

Theorem 9.3. All differential invariants for space curves under the Euclidean group are provided by the curvature $\kappa$ and either the torsion $\tau$ - for $\mathrm{SE}(3)$ - or its absolute value $|\tau|$ - for $\mathrm{E}(3)$ - along with their derivatives with respect to the signed arc length form $\left(\operatorname{sgn} \kappa_{s}\right) d s$.

Example 9.4. Consider next the case of a Euclidean surface $S \subset \mathbb{R}^{3}$, parametrized by $z(s, t)$. We use coordinates $z=(x, \widetilde{x}, u)$ on $\mathbb{R}^{3}$, and $w=(y, \widetilde{y}, v)$ for the transformed coordinates $w=R z+d$. We view $x, \widetilde{x}, y, \widetilde{y}$ as independent variables and $u, v$ as dependent variables. The standard 1-point case is well-documented in the literature, [24], and leads to the second order mean and Gaussian curvature differential invariants. Higher order differential invariants are obtained by differentiation with respect to the Frenet frame. Rather than repeat this computation here, we shall concentrate on the less well-studied case of multi-point surfaces. Let

$$
\mathbf{n}^{k}=\frac{z_{s}^{k} \wedge z_{t}^{k}}{\left\|z_{s}^{k} \wedge z_{t}^{k}\right\|}
$$

denote the unit normal at the point $z^{k}$, which is invariant, up to a sign, under reparametrizations and Euclidean transformations.

For a 2-point surface, we normalize

$$
w^{0}=0, \quad w^{1}=\lambda e_{1}, \quad v_{\tilde{y}}^{0}=0 .
$$

The translation component of the resulting left moving frame is, as usual, $\widetilde{d}=z^{0}$, while the rotation matrix becomes

$$
\widetilde{R}=\left(\mathbf{r}, \varepsilon \mathbf{t}^{0}, \sigma \varepsilon \mathbf{r} \wedge \mathbf{t}^{0}\right)
$$

where $\sigma, \varepsilon= \pm 1$, with $\sigma=+1$ in the case of $\operatorname{SE}(3)$. Here

$$
\mathbf{r}=\frac{z^{1}-z^{0}}{\left\|z^{1}-z^{0}\right\|}, \quad \text { while } \quad \mathbf{t}^{k}=\frac{\mathbf{r} \wedge \mathbf{n}^{k}}{\left\|\mathbf{r} \wedge \mathbf{n}^{k}\right\|}, \quad k=0,1
$$

are distinguished tangent directions, which requires that the surface is not orthogonal to the chord connecting $z^{0}$ to $z^{1}$ at either end. The other first order derivatives reduce to

$$
v_{y}^{0} \longmapsto-\varepsilon \frac{\mathbf{r} \cdot \mathbf{n}^{0}}{\left\|\mathbf{r} \wedge \mathbf{n}^{0}\right\|}=-\varepsilon \frac{\cos \phi^{0}}{\left|\sin \phi^{0}\right|}, \quad v_{y}^{1} \longmapsto-\sigma \frac{\mathbf{r} \cdot \mathbf{n}^{1}}{\mathbf{t}^{0} \cdot \mathbf{t}^{1}}, \quad v_{\tilde{y}}^{1} \longmapsto \sigma \frac{\mathbf{r} \cdot\left(\mathbf{n}^{0} \wedge \mathbf{n}^{1}\right)}{\mathbf{t}^{0} \cdot \mathbf{t}^{1}},
$$

where $\phi^{k}=\Varangle\left(z^{1}-z^{0}, \mathbf{n}^{k}\right)$. We require $v_{y}^{0}>0$ to fix the sign

$$
\varepsilon=-\operatorname{sgn}\left(\cos \phi^{0}\right) .
$$


We also introduce the invariant unit normals and tangents

$$
\widehat{\mathbf{n}}^{k}=\operatorname{sgn}\left(\cos \phi^{k}\right) \mathbf{n}^{k}, \quad \widehat{\mathbf{t}}^{k}=\mathbf{r} \wedge \widehat{\mathbf{n}}^{k} .
$$

The normals $\widehat{\mathbf{n}}^{k}$ are distinguished so that $0 \leq \Varangle\left(z^{1}-z^{0}, \widehat{\mathbf{n}}^{k}\right)<\frac{1}{2} \pi$. In the case of $\mathrm{E}(3)$, we can fix the sign

$$
\sigma=\operatorname{sgn}\left[\mathbf{r} \cdot\left(\widehat{\mathbf{n}}^{0} \wedge \widehat{\mathbf{n}}^{1}\right)\right]
$$

by the orientation of the chord and the two distinguished normals. The invariant differential operators are

$$
D_{y}^{k}=\sigma \frac{\left(\widehat{\mathbf{t}}^{0} \cdot z_{t}^{k}\right) D_{s}-\left(\widehat{\mathbf{t}}^{0} \cdot z_{s}^{k}\right) D_{t}}{\left(\mathbf{r} \wedge \widehat{\mathbf{t}}^{0}\right) \cdot\left(z_{s}^{k} \wedge z_{t}^{k}\right)}, \quad D_{\tilde{y}}^{k}=\frac{\left(\mathbf{r} \cdot z_{t}^{k}\right) D_{s}-\left(\mathbf{r} \cdot z_{s}^{k}\right) D_{t}}{\left(\mathbf{r} \wedge \widehat{\mathbf{t}}^{0}\right) \cdot\left(z_{s}^{k} \wedge z_{t}^{k}\right)}
$$

We therefore obtain a First Main Theorem for two-point Euclidean surfaces.

Theorem 9.5. All two-point differential invariants for Euclidean surfaces are given by

$$
\left\|z^{1}-z^{0}\right\|, \quad\left|\cos \phi^{0}\right|, \quad\left|\cos \phi^{1}\right|, \quad \widehat{\mathbf{n}}^{0} \cdot \widehat{\mathbf{n}}^{1}=\cos \psi,
$$

and, in the orientation-preserving case, the simplex volume

$$
\frac{1}{6}\left(z^{1}-z^{0}\right) \cdot\left(\widehat{\mathbf{n}}^{0} \wedge \widehat{\mathbf{n}}^{1}\right),
$$

along with their invariant derivatives. Here $\widehat{\mathbf{n}}^{k}$ are the distinguished unit normal vectors, $\psi=\Varangle\left(\widehat{\mathbf{n}}^{0}, \widehat{\mathbf{n}}^{1}\right)$ is the angle between them, and $\phi^{k}=\Varangle\left(z^{1}-z^{0}, \widehat{\mathbf{n}}^{k}\right)$.

For $n$-point surfaces with $n \geq 3$, we obtain the same joint invariants and the same moving frame as in the curve case, but the sign ambiguities are resolved slightly differently. The rotation component of the left moving frame is

$$
\widetilde{R}=R^{T}=\left(\mathbf{r}, \mathbf{n}^{\star} \wedge \mathbf{r}, \sigma \mathbf{n}^{\star}\right) .
$$

where $\sigma \pm 1$, with $\sigma=+1$ in the case of $\operatorname{SE}(3)$. Also,

$$
\mathbf{r}=\frac{z^{1}-z^{0}}{\left\|z^{1}-z^{0}\right\|}, \quad \text { while } \quad \mathbf{n}^{\star}=\frac{\left(z^{1}-z^{0}\right) \wedge\left(z^{2}-z^{0}\right)}{\left\|\left(z^{1}-z^{0}\right) \wedge\left(z^{2}-z^{0}\right)\right\|}
$$

is a unit normal to the plane through the three points $z^{0}, z^{1}, z^{2}$. The joint invariants are the interpoint distances $\left\|z^{i}-z^{j}\right\|$ and, in the case of $\mathrm{SE}(3)$, the oriented simplex volumes $V(i, j, k, l)$ (only one of which is needed). The first order differential invariants are

$$
v_{y}^{k} \longmapsto \frac{\left(\mathbf{n}^{\star} \wedge \mathbf{r}\right) \cdot \mathbf{n}^{k}}{\mathbf{n}^{\star} \cdot \mathbf{n}^{k}}, \quad v_{\tilde{y}}^{k} \longmapsto \frac{\mathbf{r} \cdot \mathbf{n}^{k}}{\mathbf{n}^{\star} \cdot \mathbf{n}^{k}},
$$

where $\mathbf{n}^{k}$ is the unit normal at $z^{k}$, cf. (9.7). For the case of $\mathrm{E}(3)$, if we have four or more points, the sign $\sigma$ can be normalized by setting it equal to the sign of the simplex volume $\sigma=\operatorname{sgn} V(0,1,2,3)$. In the three-point case, $\sigma$ is normalized by fixing the sign of one 
of the first order derivatives, say $v_{\tilde{y}}^{0}>0$. In all cases, the first and higher order joint differential invariants are obtained by invariant differentiation with respect to

$$
D_{y}^{k}=\frac{\left(\mathbf{n}^{\star} \wedge \mathbf{r}\right) \cdot z_{t}^{k} D_{s}-\left(\mathbf{n}^{\star} \wedge \mathbf{r}\right) \cdot z_{s}^{k} D_{t}}{\mathbf{n}^{\star} \cdot\left(z_{s}^{k} \wedge z_{t}^{k}\right)}, \quad D_{\tilde{y}}^{k}=\sigma \frac{\left(\mathbf{r} \cdot z_{t}^{k}\right) D_{s}-\left(\mathbf{r} \cdot z_{s}^{k}\right) D_{t}}{\mathbf{n}^{\star} \cdot\left(z_{s}^{k} \wedge z_{t}^{k}\right)} .
$$

Theorem 9.6. All $n \geq 4$ point differential invariants for Euclidean surfaces are obtained by invariantly differentiating the interpoint distances and, in the oriented case, the oriented simplex volumes. In the case $n=3$, all joint differential invariants can be found by invariantly differentiating the zero ${ }^{\text {th }}$ and first order joint differential invariants given in (9.10).

In general, there are $3 n-6$ functionally independent $n$-point joint Euclidean invariants. Therefore, one requires $3 n-6>2 n$, or $n \geq 7$ points in order to achieve at least one zero $^{\text {th }}$ order syzygy among the distance invariants, and hence a zero ${ }^{\text {th }}$ order joint signature. A first order signature can be constructed with just three points, and relies on 3 distances and 4 first order joint differential invariants. A two-point surface requires a second order signature, while the standard differential invariant signature requires the third order differential invariants arising from differentiation of the Gaussian and mean curvatures.

Example 9.7. Here we compute joint differential invariants for $n$-point space curves $C \subset \mathbb{R}^{3}$ under the equi-affine group $\mathrm{SA}(3)$. We use the same coordinates $(x, u, \widetilde{u})$ and $(y, v, \widetilde{v})=A z+d$ as in Example 9.1. We already looked at the case when the number of points $n \geq 4$ - the moving frame (3.8) leads to the zero ${ }^{\text {th }}$ order joint simplex volume invariants

$$
V(i, j, k, l)=V\left(z^{i}, z^{j}, z^{k}, z^{l}\right)=\frac{1}{6}[i j k l],
$$

where we also adapt our two-dimensional bracket notation to the three-dimensional situation now under review. For curves, the invariant differential operators are constructed by first normalizing the differentials

$$
d y^{k} \longmapsto \quad\left[\begin{array}{llll}
0 & 1 & 2 & \dot{k}
\end{array}\right] d t^{k}, \quad \text { so that } \quad \mathcal{D}_{k}=\frac{1}{\left[\begin{array}{llll}
0 & 1 & 2 & \dot{k}
\end{array}\right]} D_{t^{k}}
$$

where

$$
\left[\begin{array}{llll}
0 & 1 & 2 & \dot{k}
\end{array}\right]=\operatorname{det}\left(z^{1}-z^{0}, z^{2}-z^{0}, z_{t}^{k}\right)
$$

is the volume of the parallelepiped whose sides are the two chords connecting $z^{0}$ to $z^{1}$ and $z^{2}$ along with the tangent to the curve at $z^{k}$. Note that this is not a differential invariant - it is not even independent of the parametrization. It is not hard to prove, either directly or using the infinitesimal algorithm, that all first and higher order differential invariants are obtained from the joint simplex volume invariants by invariant differentiation. In the special case of $n=4$ points, there is just one simplex volume joint invariant, and one must differentiate twice to obtain the complete set of first order joint differential invariants; otherwise, first order derivatives suffice.

As soon as $n \leq 3$, there are no joint invariants, and we must produce the differential invariants and moving frame by normalizing the prolonged transformations. In the case of 
$n=3$ point curves, we use the cross-section

$$
w^{0}=0, \quad w^{1}=e_{3}, \quad w^{2}=e_{2}, \quad v_{y}^{0}=0, \quad \widetilde{v}_{y}^{0}=0,
$$

which implies the normalizations

$$
A Z=\operatorname{diag}(\lambda, 0,0), \quad \text { where } \quad Z=\left(z_{t}^{0}, z^{1}-z^{0}, z^{2}-z^{0}\right) .
$$

Therefore, the left moving frame is given by

$$
\widetilde{A}=\left(\frac{z_{t}^{0}}{\operatorname{det} Z}, z^{1}-z^{0}, z^{2}-z^{0}\right), \quad \widetilde{d}=z^{0}, \quad \text { where } \quad \operatorname{det} Z=\left[\begin{array}{llll}
0 & 1 & 2 & 0
\end{array}\right] .
$$

There are 4 independent first order 3-point joint differential invariants, obtained by normalizing the derivatives at $z^{1}, z^{2}$; these are the following ratios of tangent parallelepiped (or simplex) volumes:

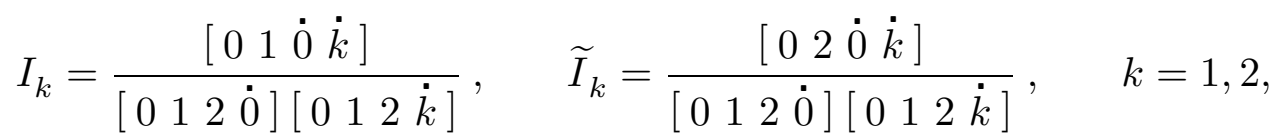

in direct analogy with the planar case (8.23). Here

$$
\left[\begin{array}{llll}
0 & 1 & 0 & \dot{k}
\end{array}\right]=\operatorname{det}\left(z^{1}-z^{0}, z_{t}^{0}, z_{t}^{k}\right) \text {. }
$$

The recurrence formulae imply that all higher order joint differential invariants are obtained by invariant differentiation with respect to $(9.13)$.

For two-point curves, we normalize

$$
w^{0}=0, \quad w^{1}=e_{3}, \quad v_{y}^{0}=0, \quad \widetilde{v}_{y}^{0}=0, \quad v_{y}^{1}=1, \quad \widetilde{v}_{y}^{1}=0, \quad v_{y y}^{0}=1,
$$

leading to the left moving frame

$$
\widetilde{A}=\left(z_{t}^{0}, z_{t}^{1}, z^{1}-z^{0}\right)\left(\begin{array}{ccc}
\lambda & -\lambda & 0 \\
0 & \mu & 0 \\
0 & 0 & 1
\end{array}\right)
$$

where

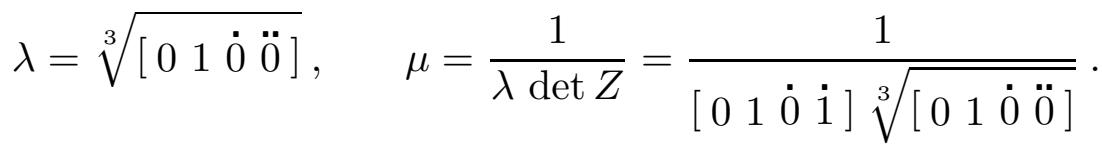

There are three second order joint differential invariants, which are found by substituting the normalizations into the remaining second order derivative coordinates. After some algebra, these are given as certain combinations of the fundamental invariants

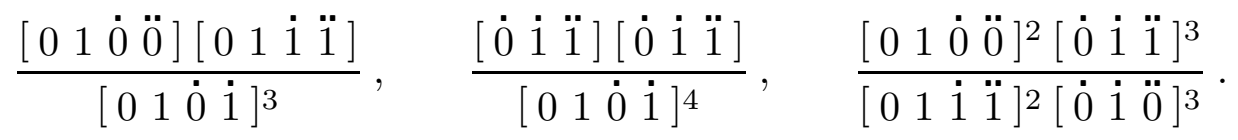

Here

$$
\left[\begin{array}{llll}
0 & 1 & 0 & 0
\end{array}\right]=\operatorname{det}\left(z^{1}-z^{0}, z_{t}^{0}, z_{t t}^{0}\right), \quad\left[\begin{array}{lll}
0 & 1 & 0
\end{array}\right]=\operatorname{det}\left(z_{t}^{0}, z_{t}^{1}, z_{t t}^{0}\right),
$$


and so on. The higher order joint differential invariants are obtained by invariant differentiation with respect to

$$
\mathcal{D}_{0}=\frac{1}{\sqrt[3]{\left[\begin{array}{llll}
0 & 1 & \dot{0} & \ddot{0}
\end{array}\right]}} D_{t^{0}}, \quad \mathcal{D}_{1}=\frac{1}{\sqrt[3]{\left[\begin{array}{llll}
0 & 1 & i & \ddot{1}
\end{array}\right]}} D_{t^{1}}
$$

Since $\operatorname{dim} \mathrm{SA}(3)=11$, there are $3 n-11$ functionally independent $n$-point joint invariants. Therefore, one requires $3 n-11>n$, or $n \geq 6$ points in order to achieve a zero $^{\text {th }}$ order syzygy among the volume invariants, and hence a zero ${ }^{\text {th }}$ order joint signature. There are $5 n-11$ independent joint differential invariants of order $\leq 1$, and so $n=3,4,5$ require first order signatures. For $n=2$, the signature is of order 2. Finally, there are 2 functionally independent fifth order equi-affine differential invariants for space curves, and the full signature curve requires their invariant (arc-length) derivatives, and so has order 6. Details can be found in $[\mathbf{2 1 , 2 4}]$.

Example 9.8. Consider finally the case of an equi-affine surface $S \subset \mathbb{R}^{3}$, parametrized by $z(s, t)$, where $z=(x, \widetilde{x}, u)$ and $w=(y, \widetilde{y}, v)=A z+d$. Let us write the left equi-affine matrix in column form, and its right inverse in row form

$$
\widetilde{A}=A^{-1}=\left(\begin{array}{lll}
\mathbf{a} \mathbf{b} \mathbf{c}
\end{array}\right), \quad A=\left(\begin{array}{c}
\mathbf{b} \wedge \mathbf{c} \\
\mathbf{c} \wedge \mathbf{a} \\
\mathbf{a} \wedge \mathbf{b}
\end{array}\right)
$$

where $\wedge$ denotes the usual wedge or cross product between column vectors, which, by convention, produces a row vector. Let

$$
[\mathbf{a} \mathbf{b} \mathbf{c}]=(\mathbf{a} \wedge \mathbf{b}) \cdot \mathbf{c}=\operatorname{det}(\mathbf{a} \mathbf{b} \mathbf{c})=1
$$

denote the standard vector triple product. The invariant differential operators can then be written as

$$
D_{y}=\frac{\left[\mathbf{c} \mathbf{a} z_{t}\right] D_{s}-\left[\mathbf{c} \mathbf{a} z_{s}\right] D_{t}}{\left[\mathbf{c} z_{s} z_{t}\right]}, \quad D_{\tilde{y}}=\frac{\left[\mathbf{c} \mathbf{b} z_{t}\right] D_{s}-\left[\mathbf{c} \mathbf{b} z_{s}\right] D_{t}}{\left[\mathbf{c} z_{s} z_{t}\right]}
$$

The first order prolonged transformation rules are then compactly written as

$$
v_{y}=-\frac{\left[\mathbf{a} z_{s} z_{t}\right]}{\left[\mathbf{c} z_{s} z_{t}\right]}, \quad v_{\tilde{y}}=-\frac{\left[\mathbf{b} z_{s} z_{t}\right]}{\left[\mathbf{c} z_{s} z_{t}\right]} .
$$

Define the quadratic form

$$
Q(\xi, \eta)=\xi^{2}\left[\begin{array}{lll}
z_{s} & z_{t} & z_{s s}
\end{array}\right]-2 \xi \eta\left[z_{s} z_{t} z_{s t}\right]+\eta^{2}\left[\begin{array}{lll}
z_{s} & z_{t} & z_{t t}
\end{array}\right],
$$

and its polarized counterpart

$$
Q\left(\xi, \eta ; \xi^{\prime}, \eta^{\prime}\right)=\xi \xi^{\prime}\left[z_{s} z_{t} z_{s s}\right]-\left(\xi \eta^{\prime}+\xi^{\prime} \eta\right)\left[z_{s} z_{t} z_{s t}\right]+\eta \eta^{\prime}\left[z_{s} z_{t} z_{t t}\right] .
$$


Then the second order prolongation formulae take the compact form

$$
\begin{gathered}
v_{y y}=\frac{Q\left(\left[\mathbf{a} \mathbf{c} z_{s}\right],\left[\mathbf{a} \mathbf{c} z_{t}\right]\right)}{[\mathbf{a} \mathbf{b} \mathbf{c}]\left[\mathbf{c} z_{s} z_{t}\right]^{3}}, \quad v_{\tilde{y} \tilde{y}}=\frac{Q\left(\left[\mathbf{b} \mathbf{c} z_{s}\right],\left[\mathbf{b} \mathbf{c} z_{t}\right]\right)}{[\mathbf{a} \mathbf{b} \mathbf{c}]\left[\mathbf{c} z_{s} z_{t}\right]^{3}} \\
v_{y \tilde{y}}=\frac{Q\left(\left[\mathbf{a} \mathbf{c} z_{s}\right],\left[\mathbf{a} \mathbf{c} z_{t}\right] ;\left[\mathbf{b} \mathbf{c} z_{s}\right],\left[\mathbf{b} \mathbf{c} z_{t}\right]\right)}{[\mathbf{a} \mathbf{b} \mathbf{c}]\left[\mathbf{c} z_{s} z_{t}\right]^{3}}
\end{gathered}
$$

Now, for an $n$-point equi-affine surface, if $n \geq 4$, then the zero ${ }^{\text {th }}$ order normalizations produce the moving frame and the joint volume invariants (9.12) computed earlier in Example 3.2. Moreover, all higher order differential invariants are obtained by invariant differentiation with respect to

$$
\mathcal{D}_{j}^{k}=\frac{\left[\begin{array}{llll}
0 & 3 & j & k_{t}
\end{array}\right] D_{s}-\left[\begin{array}{llll}
0 & 3 & j & k_{s}
\end{array}\right] D_{t}}{\left[\begin{array}{llll}
0 & 1 & 2 & 3
\end{array}\right]\left[\begin{array}{llll}
0 & 3 & k_{s} & k_{t}
\end{array}\right]}, \quad j=1,2, \quad 0=1, \ldots, k n-1,
$$

where, generalizing our earlier dot notation, $k_{t}$ stands for $z_{t}^{k}$, etc. For a three-point surface, we normalize

$$
w^{0}=0, \quad w^{1}=e_{2}, \quad w^{2}=e_{3}, \quad v_{y}^{0}=0, \quad v_{y}^{1}=0 .
$$

Note that we cannot simultaneously normalize $v_{y}^{0}$ and $v_{\tilde{y}}^{0}$ since the plane through $z^{0}, z^{1}, z^{2}$ intersects the tangent plane to the surface $\left.T S\right|_{z^{0}}$ in a line, and once we normalize $w^{0}, w^{1}, w^{2}$, this line cannot be altered. Let

$$
\mathbf{N}^{k}=z_{s}^{k} \wedge z_{t}^{k}, \quad k=0,1,
$$

denote the normals to the surface at the point $z^{k}$, and let

$$
\mathbf{T}^{\star}=\mathbf{N}^{0} \wedge \mathbf{N}^{1}
$$

denote the common tangent direction at the two points. Then the left moving frame is

$$
\widetilde{A}=\left(\lambda^{-1} \mathbf{T}^{\star}, \quad z^{1}-z^{0}, \quad z^{2}-z^{0}\right), \quad \widetilde{d}=z^{0},
$$

where

$$
\lambda=\operatorname{det}\left(\mathbf{T}^{\star}, \quad z^{1}-z^{0}, \quad z^{2}-z^{0}\right)=\mathbf{T}^{\star} \cdot \mathbf{N}^{\star}, \quad \text { where } \quad \mathbf{N}^{\star}=\left(z^{1}-z^{0}\right) \wedge\left(z^{2}-z^{0}\right)
$$

is the normal to the plane through the three points. There are four remaining first order derivatives that normalize to a complete system of first order joint differential invariants:

$$
J=\frac{\mathbf{T}^{\star} \cdot \mathbf{N}^{3}}{\left(\mathbf{T}^{\star} \cdot \mathbf{N}^{\star}\right)\left(z^{2}-z^{0}\right) \cdot \mathbf{N}^{k}}, \quad I_{k}=\frac{\left(z^{1}-z^{0}\right) \cdot \mathbf{N}^{k}}{\left(z^{2}-z^{0}\right) \cdot \mathbf{N}^{k}}, \quad k=0,1,2 .
$$

Higher order differential invariants are all obtained by invariant differentiation using

$$
\begin{aligned}
& \mathcal{D}_{k}=\frac{\left[z^{2}-z^{0}, \mathbf{T}^{\star}, z_{t}^{k}\right] D_{s}-\left[z^{2}-z^{0}, \mathbf{T}^{\star}, z_{s}^{k}\right] D_{t}}{\left(\mathbf{T}^{\star} \cdot \mathbf{N}^{\star}\right)\left(z^{2}-z^{0}\right) \cdot \mathbf{N}^{k}} \\
& \widetilde{\mathcal{D}}_{k}=\frac{\left[z^{2}-z^{0}, z^{1}-z^{0}, z_{t}^{k}\right] D_{s}-\left[z^{2}-z^{0}, z^{1}-z^{0}, z_{s}^{k}\right] D_{t}}{\left(z^{2}-z^{0}\right) \cdot \mathbf{N}^{k}} .
\end{aligned}
$$


The two point case is much more complicated, and, in view of space limitations, will be omitted. See [24] for the ordinary (1-point) differential invariants for equi-affine surfaces. For the joint signatures of an $n$-point equi-affine surface, we have $3 n-11$ independent joint invariants, and so there are $n-11$ syzygies. Therefore, one requires $n=12$ points for a purely zero ${ }^{\text {th }}$ order signature based on simplex volumes. On the other hand, a first order signature can be constructed with only $n=4$ points.

\section{Conclusions.}

In this paper, we have demonstrated the power and efficacy of the general moving frame method established in [19] for classifying joint invariants and joint differential invariants of geometric transformation groups. Our results include a wide variety of apparently new invariants and new identities, even for well-studied group actions. The syzygies (algebraic and differential identities) among joint differential invariants have been effectively classified by our infinitesimal method. Applications to the equivalence and symmetry properties of submanifolds have been developed in detail.

These investigations are motivated by applications to object recognition in digital images and the design of invariant numerical approximations to differential invariants and the theory of geometric integration, $[\mathbf{1 0}, \mathbf{2 8}]$. In numerical applications, the key is to understand how joint invariants converge to differential invariants and joint differential invariants as some or all of the points on the submanifold coalesce. Certain particular examples are known, $[\mathbf{3}, \mathbf{1 2}, \mathbf{1 3}]$, but many other important cases have yet to be properly studied. The required geometric structure, which includes the Cartesian product and prolonged jet actions in one seamless framework, will be developed in detail in a forthcoming paper.

The application of differential invariant signatures in computer vision is well-documented. Application of joint differential invariant and joint invariant signatures provide the foundation for a noise-free recognition algorithm. The key remaining ingredient is a suitable measure of the "closeness" of the joint signatures of two submanifolds. The fact that the joint signatures are constructed from very elementary joint invariants, and are no more noisy than the original image, suggests that simple least-squares comparisons might suffice for this purpose. Discrete symmetries of visual objects are readily handled by computation of the index of the signature, as described in Theorem 6.5. The signature paradigm also handles occlusions, cf. $[\mathbf{5}, \mathbf{6}, \mathbf{7}, \mathbf{1 3}]$, in a very natural fashion. The unoccluded part of the object will still produce the relevant subset of the full signature, so that, with enough of the object exposed, a sufficiently large part of the signature will suffice to uniquely characterize it among a known gallery of known object signatures. Once the signature has been recognized, one can use it to reconstruct the entire object. Details will be reported upon elsewhere.

Acknowledgments: It is a pleasure to thank Mireille Boutin, Guillermo Sapiro, Allen

Tannenbaum, and Hans Weinberger for useful remarks and encouragement. I would also like to thank the two referees for suggestions for improving the exposition. 


\section{References}

[1] Berchenko, I.A., and Olver, P.J., Symmetries of polynomials, J. Symb. Comp. 29 (2000), 485-514.

[2] Blumenthal, L.M., Theory and Applications of Distance Geometry, Oxford Univ. Press, Oxford, 1953.

[3] Boutin, M., Numerically invariant signature curves, preprint, University of Minnesota, 1999.

[4] Brown, C., Numerical evaluation of differential and semi-differential invariants, in: Geometric Invariance in Computer Vision, J.L. Mundy and A. Zisserman, eds., The MIT Press, Cambridge, Mass., 1992, pp. 215-227.

[5] Bruckstein, A.M., Holt, R.J., Netravali, A.N., and Richardson, T.J., Invariant signatures for planar shape recognition under partial occlusion, CVGIP: Image Understanding 58 (1993), 49-65.

[6] Bruckstein, A.M., Katzir, N., Lindenbaum, M., and Porat, M., Similarity invariant signatures and partially occluded planar shapes, Int. J. Comput. Vision 7 (1992), 271-285.

[7] Bruckstein, A.M., and Netravali, A.N., On differential invariants of planar curves and recognizing partially occluded planar shapes, Ann. Math. Artificial Intel. 13 (1995), 227-250.

[8] Bruckstein, A.M., Rivlin, E., and Weiss, I., Scale space semi-local invariants, Image Vision Comp. 15 (1997), 335-344.

[9] Bruckstein, A.M., and Shaked, D., Skew-symmetry detection via invariant signatures, Pattern Recognition 31 (1998), 181-192.

[10] Budd, C.J., and Iserles, A., Geometric integration: numerical solution of differential equations on manifolds, Phil. Trans. Roy. Soc. London A 357 (1999), 945-956.

[11] Carlsson, S., Mohr, R., Moons, T., Morin, L., Rothwell, C., Van Diest, M., Van Gool, L., Veillon, F., and Zisserman, A., Semi-local projective invariants for the recognition of smooth plane curves, Int. J. Comput. Vision 19 (1996), 211-236.

[12] Calabi, E., Olver, P.J., and Tannenbaum, A., Affine geometry, curve flows, and invariant numerical approximations, Adv. in Math. 124 (1996), 154-196.

[13] Calabi, E., Olver, P.J., Shakiban, C., Tannenbaum, A., and Haker, S., Differential and numerically invariant signature curves applied to object recognition, Int. J. Computer Vision 26 (1998), 107-135.

[14] Cartan, É., La Méthode du Repère Mobile, la Théorie des Groupes Continus, et les Espaces Généralisés, Exposés de Géométrie No. 5, Hermann, Paris, 1935.

[15] Cartan, É., Leçons sur la Théorie des Espaces à Connexion Projective, Cahiers Scientifiques, vol. 17, Gauthier-Villars, Paris, 1937.

[16] Dhooghe, P.F., Multilocal invariants, in: Geometry and Topology of Submanifolds, VIII, F. Dillen, B. Komrakov, U. Simon, I. Van de Woestyne, and L.

Verstraelen, eds., World Sci. Publishing, Singapore, 1996, pp. 121-137. 
[17] Faugeras, O., Cartan's moving frame method and its application to the geometry and evolution of curves in the euclidean, affine and projective planes, in: Applications of Invariance in Computer Vision, J.L. Mundy, A. Zisserman, D. Forsyth (eds.), Springer-Verlag Lecture Notes in Computer Science, Vol. 825, 1994, pp. 11-46.

[18] Fels, M., and Olver, P.J., Moving coframes. I. A practical algorithm, Acta Appl. Math. 51 (1998), 161-213.

[19] Fels, M., and Olver, P.J., Moving coframes. II. Regularization and theoretical foundations, Acta Appl. Math. 55 (1999), 127-208.

[20] Fels, M., and Olver, P.J., Moving frames and moving coframes, preprint, University of Minnesota, 1997.

[21] Green, M.L., The moving frame, differential invariants and rigidity theorems for curves in homogeneous spaces, Duke Math. J. 45 (1978), 735-779.

[22] Griffiths, P.A., On Cartan's method of Lie groups and moving frames as applied to uniqueness and existence questions in differential geometry, Duke Math. J. 41 (1974), 775-814.

[23] Gross, A.D., and Boult, T.E., Analyzing skewed symmetries, Int. J. Comput. Vision 13 (1994), 91-111.

[24] Guggenheimer, H.W., Differential Geometry, McGraw-Hill, New York, 1963.

[25] Jensen, G.R., Higher order contact of submanifolds of homogeneous spaces, Lecture Notes in Math., No. 610, Springer-Verlag, New York, 1977.

[26] Killing, W., Erweiterung der Begriffes der Invarianten von Transformationgruppen, Math. Ann. 35 (1890), 423-432.

[27] Marí-Beffa, G., and Olver, P.J., Differential invariants for parametrized projective surfaces, Commun. Anal. Geom. 7 (1999), 807-839.

[28] McLachlan, R.I., Quispel, G.R.W., and Robidoux, N., Geometric integration using discrete gradients, Phil. Trans. Roy. Soc. London A 357 (1999), 1021-1045.

[29] Menger, K., Untersuchungen über allgemeine Metrik, Math. Ann. 100 (1928), 75-163.

[30] Moons, T., Pauwels, E., Van Gool, L., and Oosterlinck, A., Foundations of semi-differential invariants, Int. J. Comput. Vision 14 (1995), 25-48.

[31] Olver, P.J., Applications of Lie Groups to Differential Equations, Second Edition, Graduate Texts in Mathematics, vol. 107, Springer-Verlag, New York, 1993.

[32] Olver, P.J., Equivalence, Invariants, and Symmetry, Cambridge University Press, Cambridge, 1995.

[33] Olver, P.J., Classical Invariant Theory, London Math. Soc. Student Texts, vol. 44, Cambridge University Press, Cambridge, 1999.

[34] Olver, P.J., Moving frames and singularities of prolonged group actions, Selecta Math. 6 (2000), 41-77.

[35] Ovsiannikov, L.V., Group Analysis of Differential Equations, Academic Press, New York, 1982. 
[36] Pauwels, E., Moons, T., Van Gool, L.J., Kempenaers, P., and Oosterlinck, A., Recognition of planar shapes under affine distortion, Int. J. Comput. Vision 14 (1995), 49-65.

[37] Strang, G., Linear Algebra and its Applications, Third Ed., Academic Press, San Diego, 1988.

[38] Van Gool, L., Brill, M.H., Barrett, E.B., Moons, T., and Pauwels, E., Semi-differential invariants for nonplanar curves, in: Geometric Invariance in Computer Vision, J.L. Mundy and A. Zisserman, eds., The MIT Press, Cambridge, Mass., 1992, pp. 293-309.

[39] Van Gool, L., Moons, T., Pauwels, E., and Oosterlinck, A., Semi-differential invariants, in: Geometric Invariance in Computer Vision, J.L. Mundy and A. Zisserman, eds., The MIT Press, Cambridge, Mass., 1992, pp. 157-192.

[40] Van Gool, L., Moons, T., and Proesmans, M., Mirror and point symmetry under perspective skewing, in: Proceedings IEEE Computer Society Conference on Computer Vision and Pattern Recognition, IEEE Press, Los Alamitos, CA, 1996, pp. 285-292.

[41] Van Gool, L., Moons, T., Ungureanu, D., and Oosterlinck, A., The characterization and detection of skewed symmetry, Computer Vision Image Understanding $\mathbf{6 1}$ (1985), 138-150.

[42] Veblen, O., and Young, J.W., Projective Geometry, vol. 2, Blaisdell Publ. Co., New York, 1946.

[43] Weyl, H., Classical Groups, Princeton Univ. Press, Princeton, N.J., 1946. 